University of Tennessee Health Science Center

UTHSC Digital Commons

\title{
Revealing a Non-canonical Role of Anti-apoptotic MCL-1 in Early Embryonic Development
}

\author{
Xue Yang \\ University of Tennessee Health Science Center
}

Follow this and additional works at: https://dc.uthsc.edu/dissertations

Part of the Genetic Phenomena Commons, Medical Cell Biology Commons, Medical Genetics Commons, and the Neoplasms Commons

\section{Recommended Citation}

Yang, Xue (http://orcid.org/0000-0001-8557-4342), "Revealing a Non-canonical Role of Anti-apoptotic MCL-1 in Early Embryonic Development" (2017). Theses and Dissertations (ETD). Paper 451.

http://dx.doi.org/10.21007/etd.cghs.2017.0443.

This Dissertation is brought to you for free and open access by the College of Graduate Health Sciences at UTHSC Digital Commons. It has been accepted for inclusion in Theses and Dissertations (ETD) by an authorized administrator of UTHSC Digital Commons. For more information, please contact jwelch30@uthsc.edu. 


\title{
Revealing a Non-canonical Role of Anti-apoptotic MCL-1 in Early Embryonic Development
}

\author{
Abstract \\ MCL-1, a well-known pro-survival BCL-2 family member, is indispensable for the survival of various cellular \\ lineages and is also among the most frequently amplified genes in a variety of human malignancies. Gene \\ ablation studies previously revealed that $\mathrm{Mcl}-1$ deficiency leads to embryonic lethality around E3.5 during \\ peri-implantation stage. Strikingly, the study did not detect any increase in apoptotic cells of the \\ blastocyst, indicating a function of MCL-1 beyond regulating apoptosis. Our previous studies revealed an \\ unrecognized role of MCL-1 in promoting mitochondrial physiology, which is independent of its classical \\ anti-apoptotic function and requires being imported into the mitochondrial matrix. In order to understand \\ the role of MCL-1 in early embryonic development, we used CRISPR-Cas 9 to target Mcl-1's start codon on \\ established embryonic stem cells (ESCs). This approach resulted in the establishment of ESCs in which \\ MCL-1's N-terminus was truncated. Biochemical evaluation revealed that Nterminal- deleted MCL-1 \\ retains anti-apoptotic function. However, this truncated MCL-1 is restricted to the mitochondrial outer \\ membrane and functionally these mutated ESCs showed a dramatic defect in differentiation into the three \\ embryonic germ layers- ectoderm, mesoderm, and ectoderm. These data suggest that in addition to \\ MCL-1's required antagonism of cell death by the C-terminal region, MCL-1's N-terminus is required for \\ efficient cellular differentiation, potentially by facilitating MCL-1's import into the mitochondrial matrix. \\ Document Type \\ Dissertation \\ Degree Name \\ Doctor of Philosophy (PhD) \\ Program \\ Biomedical Sciences \\ Research Advisor \\ Joseph T. Opferman, Ph.D. \\ Keywords \\ Anti-apoptosis, BCL-2 family, CRISPR-Cas9, MCL-1, Non-canonical role, Stem cell differentiation

\section{Subject Categories} \\ Genetic Phenomena | Medical Cell Biology | Medical Genetics | Medical Sciences | Medicine and Health \\ Sciences | Neoplasms
}


Revealing a Non-canonical Role of Anti-apoptotic MCL-1 in Early Embryonic Development

\author{
A Dissertation \\ Presented for \\ The Graduate Studies Council \\ The University of Tennessee \\ Health Science Center
}

In Partial Fulfillment

Of the Requirements for the Degree

Doctor of Philosophy

From The University of Tennessee

By

Xue Yang

December 2017 
Copyright (C) 2017 by Xue Yang. All rights reserved. 


\section{DEDICATION}

This dissertation is dedicated to every child at St. Jude Children's Research

Hospital who is fighting cancer. Their courage and braveness inspire and motivate me to become a better person, do a better job and create a better world. 


\section{ACKNOWLEDGEMENTS}

I am sincerely thankful to my research advisor, Dr. Joseph Opferman, who supported and tutored me to conduct research projects throughout my $\mathrm{Ph}$.D. His patient guidance, diligent working attitude and scientific logic have led me through the darkness of my study and will continuously encourage me in the future. I would also thank everyone in the Opferman laboratory, Brian Koss, Madhavi Bathina, Haiyan Xu, Drs. Rhonda Perciavalle, Amit Budhraja, Xi Wang, Christopher Mill, Katherine Szarama, Ewa Kaminska, and Meghan Turnis for technical assistance and helpful suggestions.

I would like to take this opportunity to extend my gratitude to my committee, Drs. Zambetti, Roussel, Kundu, and Nelson for their support, supervision and assistance during the past several years. Thank them for inspiring and helping me to expand my knowledge and background in cancer and developmental biology.

My deepest thank to Drs. Chunliang Li and Charles Sherr for the greatest collaboration and their generously devoted time and expertise to broaden my view in the field. I want to especially thank Dr. Chunliang Li and his people for their help, discussion and technical support to my research.

I owe a great deal of gratitude to my husband and my parents for their neverending and unconditional love which is the greatest motivation in my way to be a better self.

Finally, none of this would happen without the support of the University of Tennessee Health Science Center, St. Jude Children's Research Hospital, ALSAC and NIH. As a student scientist, I am particularly grateful for the opportunities provided. 


\begin{abstract}
MCL-1, a well-known pro-survival BCL-2 family member, is indispensable for the survival of various cellular lineages and is also among the most frequently amplified genes in a variety of human malignancies. Gene ablation studies previously revealed that Mcl-1 deficiency leads to embryonic lethality around E3.5 during peri-implantation stage. Strikingly, the study did not detect any increase in apoptotic cells of the blastocyst, indicating a function of MCL-1 beyond regulating apoptosis. Our previous studies revealed an unrecognized role of MCL-1 in promoting mitochondrial physiology, which is independent of its classical anti-apoptotic function and requires being imported into the mitochondrial matrix. In order to understand the role of MCL-1 in early embryonic development, we used CRISPR-Cas9 to target $\mathrm{Mcl}$-1's start codon on established embryonic stem cells (ESCs). This approach resulted in the establishment of ESCs in which MCL-1's N-terminus was truncated. Biochemical evaluation revealed that Nterminal-deleted MCL-1 retains anti-apoptotic function. However, this truncated MCL-1 is restricted to the mitochondrial outer membrane and functionally these mutated ESCs showed a dramatic defect in differentiation into the three embryonic germ layersectoderm, mesoderm, and ectoderm. These data suggest that in addition to MCL-1's required antagonism of cell death by the C-terminal region, MCL-1's N-terminus is required for efficient cellular differentiation, potentially by facilitating MCL-1's import into the mitochondrial matrix.
\end{abstract}




\section{TABLE OF CONTENTS}

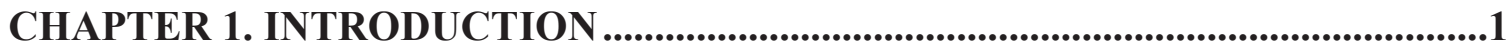

The BCL-2 Family and Apoptosis .....................................................................

Myeloid Cell Leukemia-1 ....................................................................................4

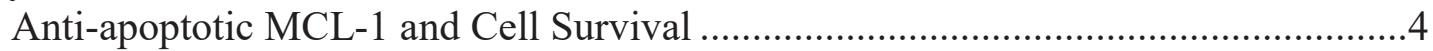

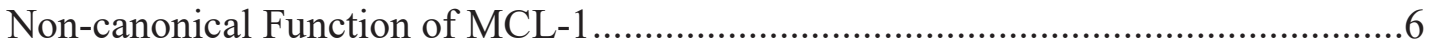

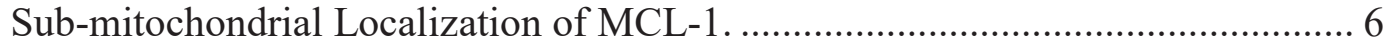

MCL-1 Possesses Multiple Functions in Mitochondria........................................ 7

Mitochondria in Human Development and Diseases ...............................................9

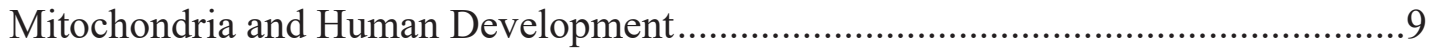

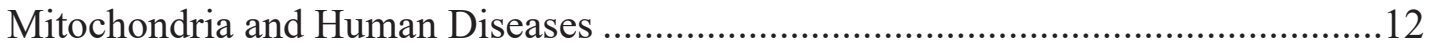

Human Diseases Associated with MtDNA Mutations...................................... 12

Human Diseases from Mutations in Mitochondrial Function Related Nuclear

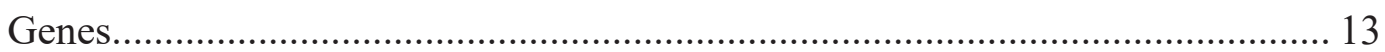

Mitochondria and Cancer................................................................................. 13

MtDNA Mutations and Cancer...................................................................... 13

Mitochondrial Signaling Pathway and Cancer. ................................................ 13

Oxidative Stress and Cancer. ........................................................................ 14

Targeting Mitochondria for Cancer Therapy................................................ 15

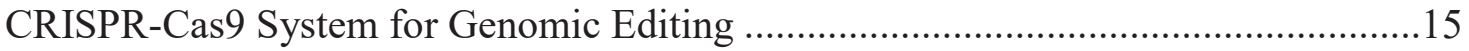

CHAPTER 2. MATERIALS AND EXPERIMENTAL PROCEDURES ...................18

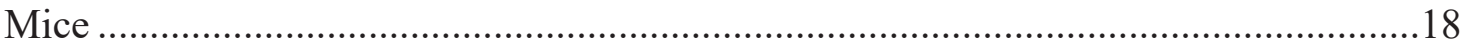

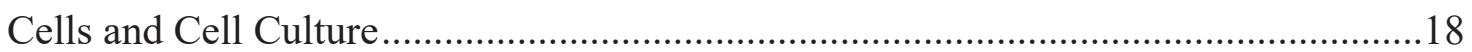

Plasmids, Expression Constructs, and Generation of Mutants ...................................19

Ecotropic Retroviral Production and Cell Transduction............................................19

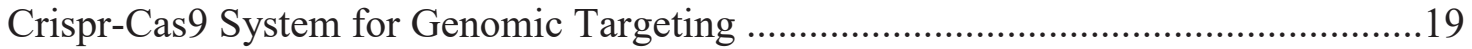

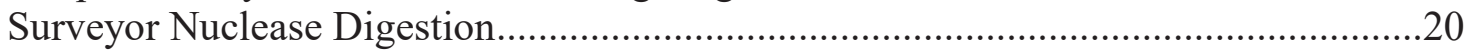

Immunoblotting, Co-immunoprecipitation, and Antibodies.......................................20

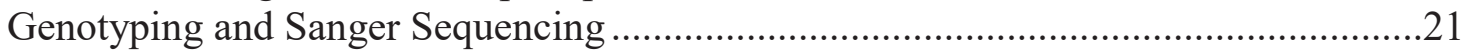

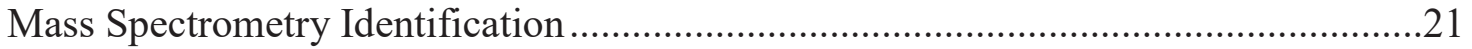

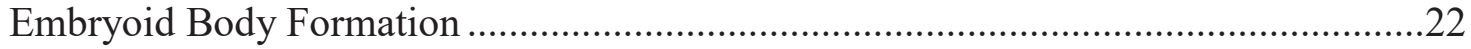

Cryosectioning and Immunofluorescent Staining of Embryoid Bodies ......................22

RNA Isolation and Quantification ......................................................................22

Teratoma Formation Assay ......................................................................................23

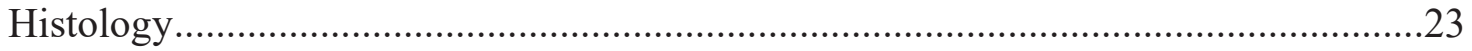

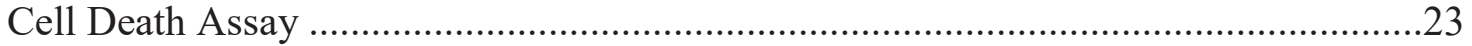

Subcellular Fractionation and Proteolysis of Heavy Membrane Fraction ....................23

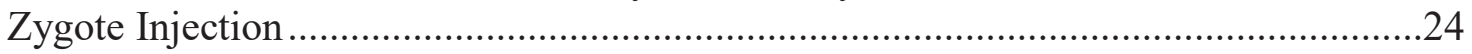

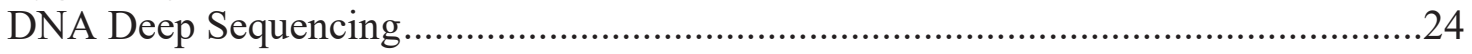

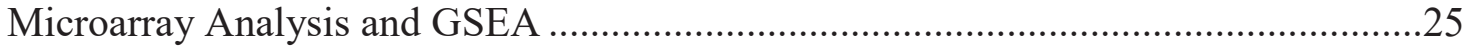

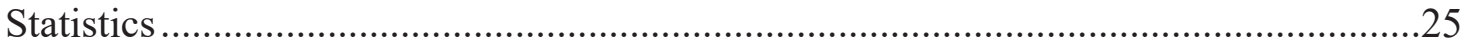


CHAPTER 3. RESULTS .26

CRISPR-Cas9 Targeting of Genomic Mcl-1 to Generate $M c l-1$ Mutants .26

Mutant ESCs Show a "Sick" Phenotype and Fail to Differentiate to Broad Lineages..29 Mutant MCL-1 Is Capable of Antagonizing Cell Death and Localizes to the Outer

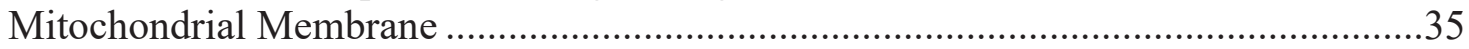

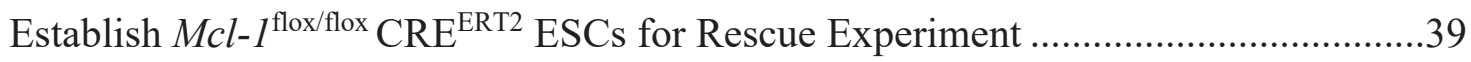

Zygote Injection to Generate MCL-1 Mutant Mice......................................................45

CHAPTER 4. DISCUSSION ...........................................................................................53

MCL-1 in Normal Biology ………………………..............................................53

MCL-1 in Embryonic Development …………………......................................53

Metabolic Requirements during Embryogenesis............................................... 54

Metabolic Determinants of Embryonic Stem Cell Fate........................................ 54

MCL-1 in Cardiac Homeostasis............................................................................56

Mitochondrial Function in Cardiomyocyte Development. ................................... 56

MCL-1's Dual Functions in Heart Homeostasis....................................................... 56

MCL-1 in Hematopoiesis ..................................................................................57

MCL-1 in Cancer Biology and Cancer Therapy ………..........................................57

Targeting MCL-1 as an Anti-apoptotic Protein ......................................................58

Targeting Matrix-MCL-1 for Cancer Therapy..........................................................58

Potential Concerns of Targeting MCL-1 in Cancer Therapies ...................................59

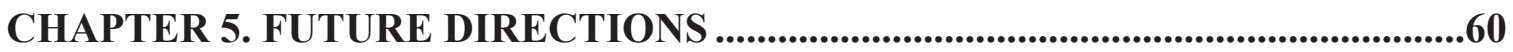

Contributions of Both MCL-1 Species for ESCs Survival and Differentiation ............60

Differentiation Rescue Experiments by Enforced Expression of MCL-1 Species ........60

Determine Mechanistically How Both Forms of MCL-1 Regulate ESCs

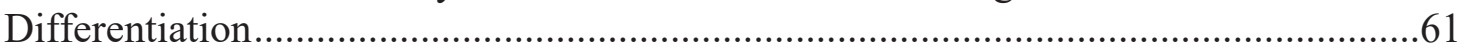

Determine Physiological Mitochondrial Status of Mutant ESCs ...............................61

Determine Mechanistically How MCL-1 Regulates ESCs Differentiation ................61

Determine the Phenotypes of $\mathrm{CS}^{10,11}$ and $30 \mathrm{kD}$ MCL-1 KI Mice.............................66

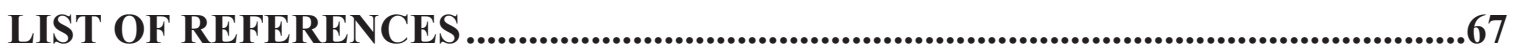

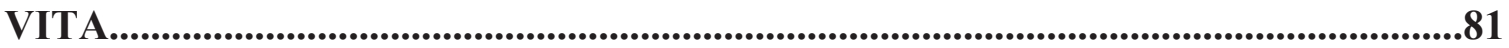




\section{LIST OF TABLES}

Table 3-1. Summary of F12 ESCs transfected with CRISPR-Cas9 targeting on

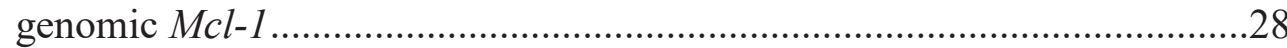




\section{LIST OF FIGURES}

Figure 1-1. Scheme depicting intrinsic and extrinsic arms of apoptosis .......................2

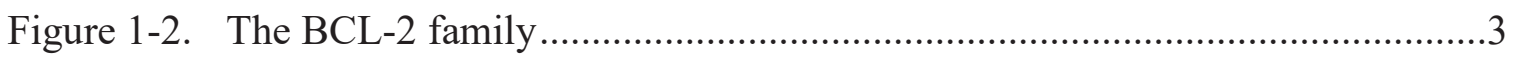

Figure 1-3. Role of anti-apoptotic regulators during hematopoiesis .............................5

Figure 1-4. Model for MCL-1's potential functions in mitochondria ...........................

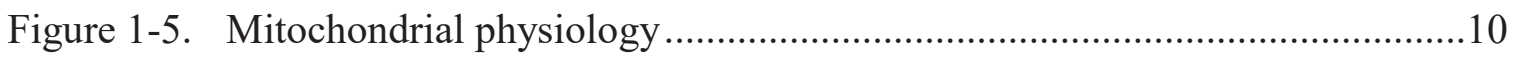

Figure 1-6. Schematic of the RNA-guided Cas9 nuclease ......................................... 17

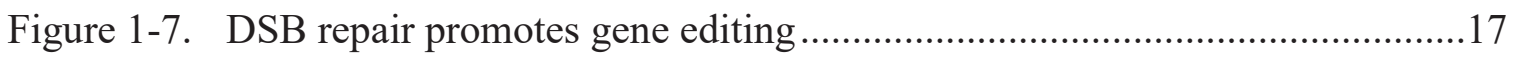

Figure 3-1. Pool validation of CRISPR-Cas9 targeting efficiency .............................27

Figure 3-2. Mass spectrometry N-terminal identification of mutant MCL-1 _................30

Figure 3-3. Mutant ESCs present a "sick" phenotype in culture ...................................32

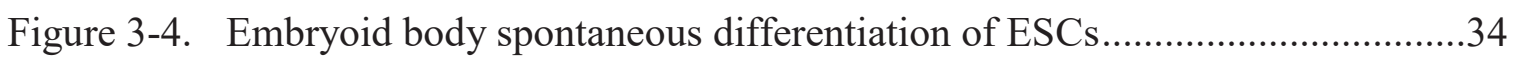

Figure 3-5. $30 \mathrm{kD}$ mutant ESCs show broad differentiation defects ............................36

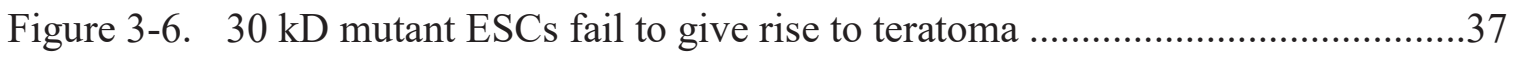

Figure 3-7. $30 \mathrm{kD}$ mutant ESCs retain anti-apoptotic function and reside on the outer

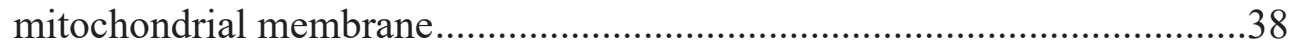

Figure 3-8. Generation of inducible MEFs overexpressing $30 \mathrm{kD}$ mutant for cell death and subcellular localization analyses...........................................40

Figure 3-9. Mcl-1-deleted $M c l-1^{\text {flox/flox }} \mathrm{CRE}^{\text {ERT2 }} \mathrm{ESCs}$ fail to proliferate in vitro and give rise to teratoma in vivo ..........................................................4 41

Figure 3-10. Dissect MCL-1's two isoforms for rescue experiments ............................43

Figure 3-11. Put back MCL-1 mutants for proliferation rescue ...................................44

Figure 3-12. Teratoma formation needs both forms of MCL-1 .................................46

Figure 3-13. Deep sequencing shows mutations and mutation efficiencies in founders

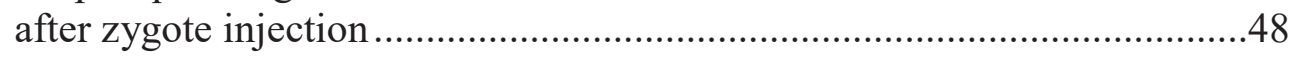

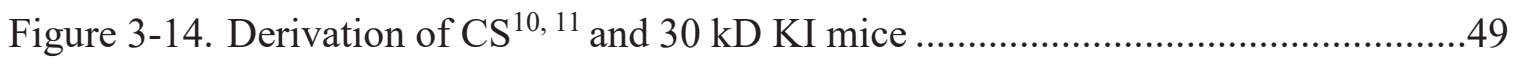


Figure 3-15. Validation of $\mathrm{CS}^{10,11}$ and $30 \mathrm{kD} \mathrm{KI}$ mice

Figure 5-1. MCL-1 expression is first induced and then decreased during differentiation process

Figure 5-2. Potential changes of signaling pathways are identified in $\mathrm{Mcl}$-1-deleted ESCs 


\section{LIST OF ABBREVIATIONS}

2DG

A1

ADP

AIF

ALL

AML

ANTs

APAF-1

ATP

ATP5B

BAD

BAK

BAX

BCL-2

BCL-W

BCL- $X_{L}$

$\mathrm{BH}$

BID

BIM

BMI-1

BMP

BOK

BSA

CLL

CLPs

CML

CMPs

$\mathrm{CoA}-\mathrm{SH}$

CoQ

CPEO

CRISPR

crRNA

CS

$\mathrm{CS}^{10,11}$ MCL-1

$\mathrm{CS}^{10,11} \mathrm{KI}$

CSC

C-terminal

$\mathrm{Cu} / \mathrm{ZnSOD}$

CYPD

DAB-2

DAPI

DMEM

DRP1
2-deoxy-D-glucose

BCL-2 related protein A1

Adenosine diphosphate

Apoptosis-inducing factor

Acute lymphoblastic leukemia

Acute myelogenous leukemia

Adenine nucleotide translocators

Apoptotic protease activating factor-1

Adenosine triphosphate

Mitochondrial ATP synthase beta subunit

BCL-2 antagonist of cell death

BCL-2 antagonist/killer

BCL-2-associated protein

B cell lymphoma 2

BCL-2-like protein 2

BCL-2-like protein 1

BCL-2 homology

BH3-interacting-domain death agonist

BCL-2-interacting mediator of cell death

$\mathrm{B}$ cell-specific Moloney murine leukemia virus integration site 1

Bone morphogenetic protein

BCL-2-related ovarian killer

Bovine serum albumin

Chronic lymphocytic leukemia

Common lymphoid progenitors

Chronic myelogenous leukemia

Common myeloid progenitors

Coenzyme A with a free sulphydryl group

Coenzyme Q10

Chronic progressive external ophthalmoplegia

Clustered regularly interspaced short palindromic repeat

CRISPR RNA

Cleavage site

MCL-1 amino acids 10, 11 replacement mutant

Cleavage site amino acid 10, 11 knock-in

Cancer stem cells

Carboxyl-terminal

$\mathrm{Cu} / \mathrm{Zn}$ superoxide dismutase

Cyclophilin D

Disabled homolog 2

4',6-diamidino-2-phenylindole

Dulbecco's modified Eagle's medium

Dynamin-related protein 1 


\begin{tabular}{|c|c|}
\hline DSBs & Double-strand breaks \\
\hline $\mathrm{E}$ & Embryonic day \\
\hline EB & Embryoid body \\
\hline EDTA & Ethylene diaminetetraacetic acid \\
\hline EED & Embryonic ectoderm development \\
\hline EMT & Epithelial to mesenchymal transition \\
\hline EMX1 & Empty spiracles homeobox 1 \\
\hline ENDOG & Mitochondrial endonuclease $\mathrm{G}$ \\
\hline ESCs & Embryonic stem cells \\
\hline ETC & Electron transport chain \\
\hline EZH2 & Enhancer of zeste homolog 2 \\
\hline FBS & Fetal bovine serum \\
\hline FGF5 & Fibroblast growth factor 5 \\
\hline $\mathrm{FM}$ & Fast-mobility \\
\hline GATA4 & GATA binding protein 4 \\
\hline GMP & Granulocyte monocyte progenitor \\
\hline GPX & Glutathione peroxidase \\
\hline GSEA & Gene set enrichment analysis \\
\hline $\mathrm{H}_{2} \mathrm{O}_{2}$ & Hydrogen peroxide \\
\hline H3К27 & Lysine 27 of histone $\mathrm{H} 3$ \\
\hline $\mathrm{H} \& \mathrm{E}$ & Hematoxylin and eosin \\
\hline HEK & Human embryonic kidney \\
\hline HDR & Homology-directed repair \\
\hline HM & Heavy membrane \\
\hline HSC & Hematopoietic stem cell \\
\hline ICMs & Inner cell masses \\
\hline Indels & Insertions or deletions \\
\hline IF & Immunofluorescent \\
\hline $\mathrm{IHH}$ & Indian hedgehog \\
\hline IL & Interleukin \\
\hline IMM & Inner mitochondrial membrane \\
\hline IP & Immunoprecipitation \\
\hline iPS & Induced pluripotent stem cell \\
\hline $\mathrm{kD}$ & Kilodalton \\
\hline KI & Knock-in \\
\hline KSS & Kearns-Sayre Syndrome \\
\hline $\mathrm{LDH}$ & Lactate dehydrogenase \\
\hline LHON & Leber's hereditary optic neuropathy \\
\hline LIF & Leukemia inhibitory factor \\
\hline $\mathrm{m} / \mathrm{z}$ & Mass-to-charge ratio \\
\hline MCL-1 & Myeloid cell leukemia-1 \\
\hline MEFs & Mouse embryonic fibroblasts \\
\hline MEP & Megakaryocyte erythroid progenitor \\
\hline MET & Mesenchymal to epithelial transition \\
\hline $\mathrm{MFN}$ & Mitofusin \\
\hline MIB & Mitochondria isolation buffer \\
\hline
\end{tabular}




\begin{tabular}{|c|c|}
\hline MIG & MSCV-I-GFP \\
\hline MnSOD & Mn superoxide dismutase \\
\hline MOCO1 & Mutation of cytochrome c oxidase subunit 1 \\
\hline MPP & Mitochondrial processing peptidase \\
\hline MPPs & Multipotent progenitors \\
\hline MPTP & Mitochondrial permeability transition pore \\
\hline MS & Mass spectrometry \\
\hline MSCs & Mesenchymal stem cells \\
\hline MSCV & Murine stem cell virus \\
\hline MTCO1 & Mitochondrial cytochrome $c$ oxidase subunit 1 \\
\hline MTND3 & NADH dehydrogenase subunit 3 \\
\hline mtDNA & Mitochondrial DNA \\
\hline mTOR & Mechanistic target of rapamycin \\
\hline mtPTP & Mitochondrial permeability transition pore \\
\hline NAC & $\mathrm{N}$-acetyl cysteine \\
\hline NADPH & Nicotinamide adenine dinucleotide phosphate \\
\hline ND6 & NADH dehydrogenase subunit 6 \\
\hline NHEJ & Non-homologous end joining \\
\hline NK & Natural killer \\
\hline N-terminal & Amino-terminal \\
\hline $\mathrm{O}_{2}^{\cdot-}$ & Superoxide \\
\hline OAA & Oxaloacetate \\
\hline OCRs & Oxygen consumption rates \\
\hline OCT-4 & Octamer-binding transcription factor 4 \\
\hline$\cdot \mathrm{OH}$ & Hydroxyl free radicals \\
\hline OMM & Outer mitochondrial membrane \\
\hline OPA-1 & Optic Atrophy Protein 1 \\
\hline OXPHOS & Oxidative phosphorylation \\
\hline PAM & Protospacer adjacent motif \\
\hline PARP & Poly ADP ribose polymerase \\
\hline $\mathrm{PB}$ & Polybrene \\
\hline PBS & Phosphate-buffered saline \\
\hline PCA & Principal components analysis \\
\hline PcG & Polycomb group \\
\hline PDH & Pyruvate dehydrogenase \\
\hline PFA & Paraformaldehyde \\
\hline PIC & Protease inhibitor cocktail \\
\hline PK & Proteinase K \\
\hline PRC & Polycomb repressive complexes \\
\hline PT & Pyruvate transporter \\
\hline PTEN & Phosphatase and tensin homolog \\
\hline PUMA & P53-upregulated modulator of apoptosis \\
\hline PVDF & Polyvinylidene fluoride \\
\hline qPCR & Quantitative real-time PCR \\
\hline $\mathrm{RBCs}$ & Red blood cells \\
\hline RIPA & Radioimmunoprecipitation assay \\
\hline
\end{tabular}


RNAi

ROS

RT

SDS

SDS-PAGE

SEM

SMAC

SOD2

SOX7

ssODNs

STAT3

SUZ12

SV40

TALE

TALENs

TCA

TE

TICs

TIM

TNF

TNFR1

TOM

TPA

TSC1

TSPO

UTR

VDACs

WCLs

WT

ZFNs
RNA-mediated interference

Reactive oxygen species

Room temperature

Sodium deoxycholate

Sodium dodecyl sulfate poyacryamide gel electrophoresis

Standard error of the mean

Second mitochondria-derived activator of caspase

Superoxide dismutase

Sry-related high-mobility group box 7

Single-stranded DNA oligonucleotides

Signal transducer and activator of transcription 3

Suppressor of zeste 12

Simian virus 40

Transcription activator-like effector

Transcription activator-like effector nucleases

Tricarboxylic acid

Trophectoderm

Tumor-initiating cells

Translocases of inner membrane

Tumor necrosis factor

Tumor necrosis factor receptor-1

Translocases of outer membrane

12-O-tetradecanoylphorbol-13-acetate

Tuberous sclerosis complex 1

Translocator protein

Untranslated region

Voltage-dependent anion channels

Whole cell lysates

Wild-type

Zinc-finger nucleases 


\section{CHAPTER 1. INTRODUCTION}

\section{The BCL-2 Family and Apoptosis}

Apoptosis, or programmed cell death, is important for the proper development and maintenance of homeostasis throughout the life of all mammals. Dysregulation of this genetic program leads to human diseases including cancers, autoimmune diseases, and neurodegenerative disorders [1]. Apoptosis is initiated by cell death stimuli and converges on the activation of caspases, and cysteinyl aspartate proteases which cleave protein substrates within the doomed cells to trigger the apoptotic process [2]. Two death pathways can be distinguished by whether BCL-2 family is required and which caspases are involved [3] (Figure 1-1).

The extrinsic or death-receptor pathway is triggered by the aggregation of death receptors (members of the tumor necrosis factor (TNF) receptor family, such as Fas or TNF receptor-1 (TNFR1)) upon extracellular ligand stimulation. The death receptors recruit and activate the initiator caspase-8, causing subsequent recruitment of downstream caspase-3, -6 , or -7 , without requirement of the BCL-2 family. In certain cell types, most notably hepatocytes, the extrinsic pathway can intersect the intrinsic pathway through capsase- 8 cleavage-mediated activation by pro-apoptotic $\mathrm{BH} 3$-only protein BID [2] (Figure 1-1).

On the other hand, the intrinsic pathway, or mitochondrial pathway, is strictly regulated by the BCL-2 family members [3]. The BCL-2 family is composed of antiapoptotic and pro-apoptotic members that share homology with four conserved amphipathic alpha helical segments named BCL-2 homology domains $(\mathrm{BH})$ numbered BH1-4 [2]. Anti-apoptotic members (such as BCL-2, BCL-X, , MCL-1, A1 and BCL-w) possess all four $\mathrm{BH}$ domains and form a hydrophobic pocket capable of binding the $\mathrm{BH} 3$ domains of other pro-apoptotic members to antagonize apoptosis. The pro-apoptotic members can be further subdivided according to the number of $\mathrm{BH}$ domains they possess; multidomain pro-apoptotic members (BAX, BAK and BOK) and the divergent subset of BH3-only members (BAD, BIK, BID, BIM, NOXA, PUMA, etc.) [4-6] (Figure 1-2). The central dogma for the BCL-2 family in apoptosis is that death signals trigger the activation of $\mathrm{BH} 3$-only proteins which can promote the oligomerization of $\mathrm{BAX}$ and BAK. BAX and BAK oligomers permeabilize the outer mitochondrial membrane (OMM) and induce the release of several apoptogenic proteins including cytochrome $c$. Once cytochrome $c$ is released, it binds to the cytosolic protein apoptotic protease-activating factor-1 (APAF-1) to facilitate the formation of apoptosome which can recruit and activate pro-caspase-9. Activated initiator caspase- 9 further activates effector caspases and triggers a cascade of events leading to apoptosis [7] (Figure 1-1). 


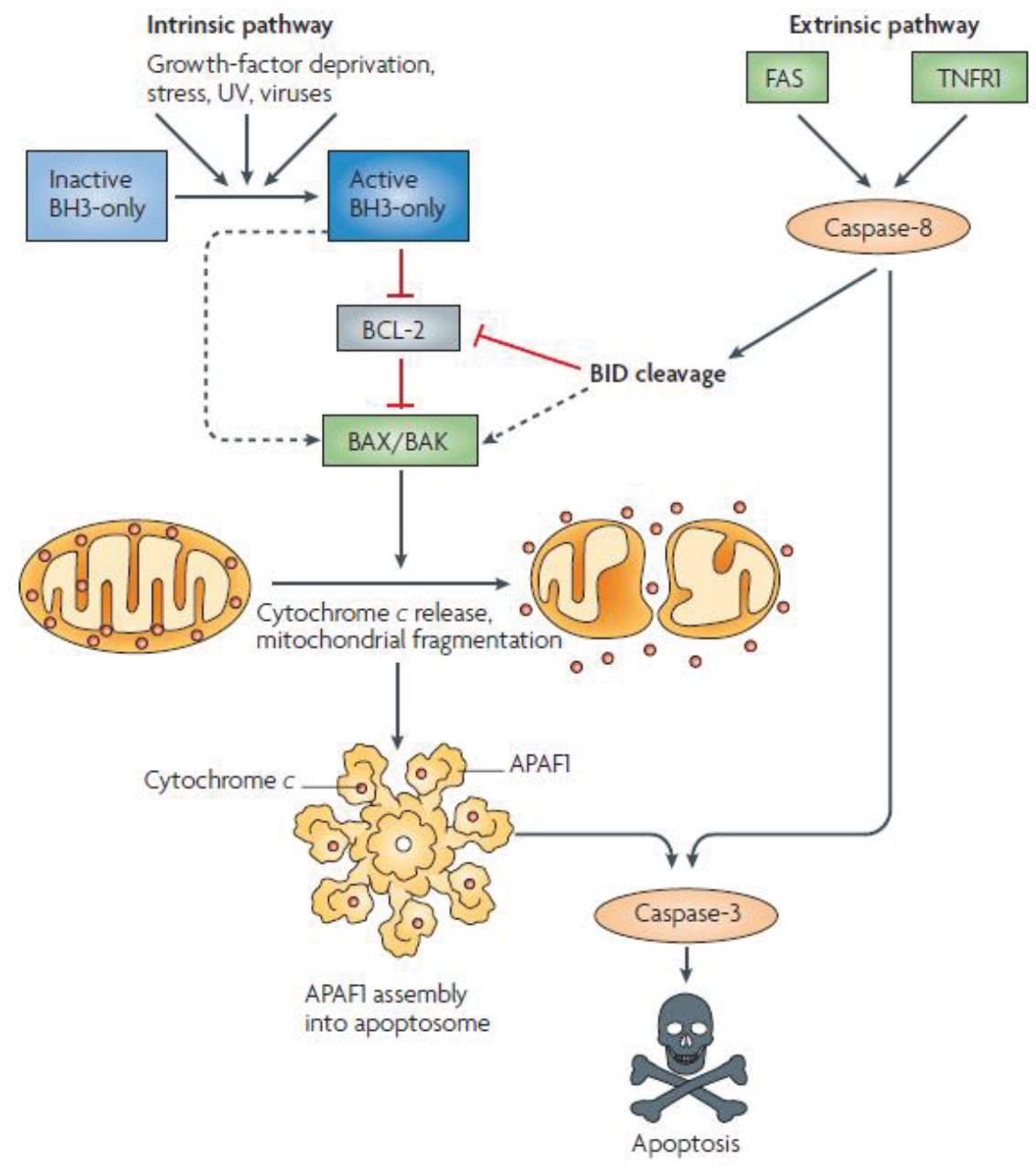

Figure 1-1. Scheme depicting intrinsic and extrinsic arms of apoptosis Apoptosis can be induced by cell surface receptors, such as FAS and tumor necrosis factor receptor-1 (TNFR1) (extrinsic pathway, right), or by various genotoxic agents, metabolic insults or transcriptional cues (intrinsic pathway, left). The intrinsic pathway starts with $\mathrm{BH} 3$-only protein induction or post-translational activation, which results in the inactivation of some BCL-2 family members. This relieves inhibition of BAX and BAK, which in turn promotes apoptosis. Some BH3-only proteins, such as BIM and PUMA, may also be able to activate BAX and/or BAK (as shown by the dotted line). Once activated, BAX and BAK promote cytochrome $c$ release and mitochondrial fission, which leads to the activation of APAF-1 into an apoptosome and activates caspase-9 to activate caspase-3. Caspases in turn cleave a series of substrates, activate DNAses and orchestrate the demolition of the cell. The extrinsic pathway can bypass the mitochondrial step and activate caspase- 8 directly, which leads to caspase- 3 activation and cell demolition. The BCL-2 family regulates the intrinsic pathway and can modulate the extrinsic pathway when cleavage of BID communicates between the two pathways. Reprinted with permission from Macmillan Publishers Ltd: Youle RJ, Strasser A. 2008. The BCL-2 protein family: opposing activities that mediate cell death. Nat Rev Mol Cell Biol 9: 47-59. 


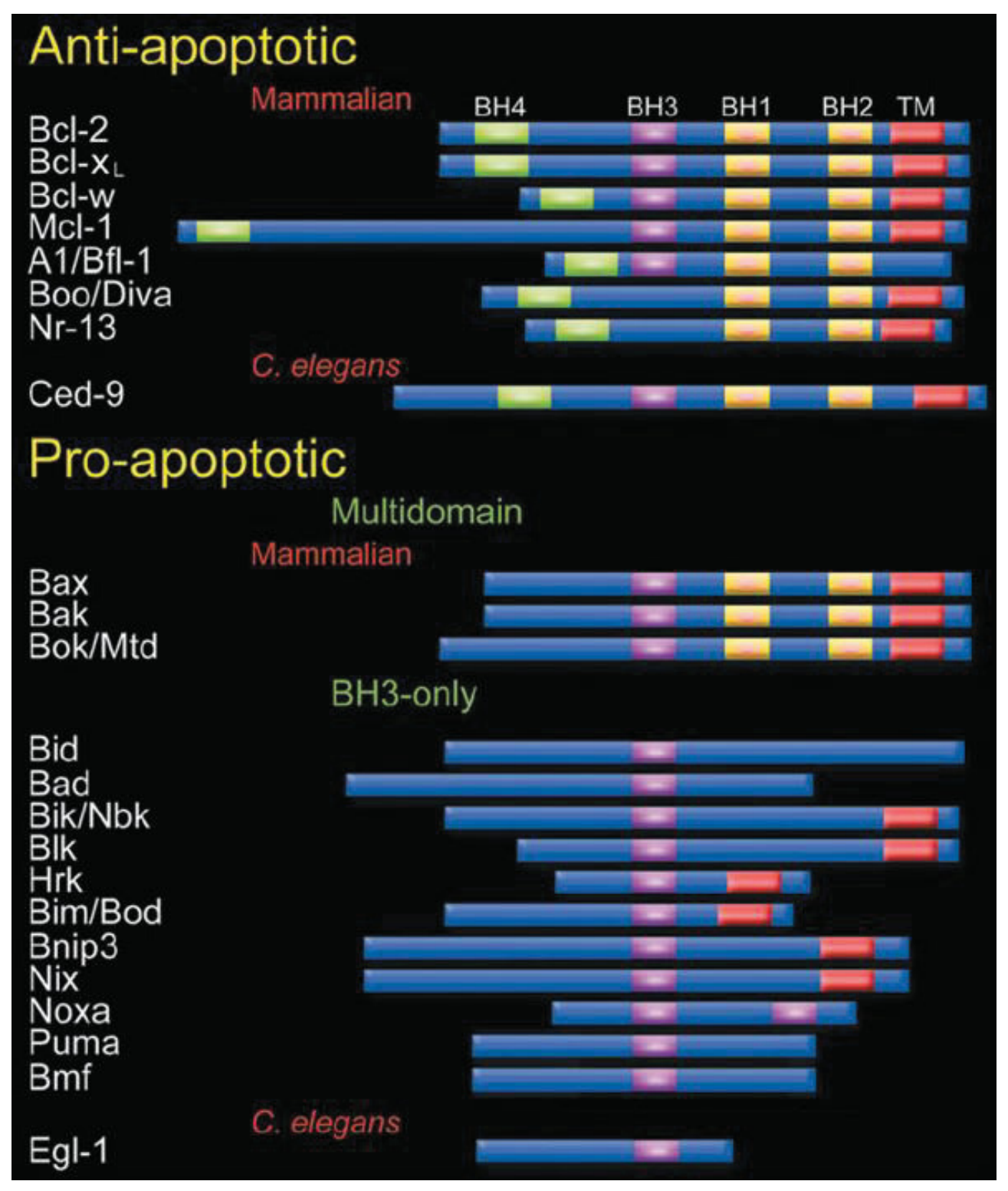

\section{Figure 1-2. The BCL-2 family}

The BCL-2 family comprises pro-apoptotic and anti-apoptotic members that share homology with four conserved amphipathic alpha helical segments named BH1-BH4. The highly conserved anti-apoptotic family members (BCL-2, BCL-X $\mathrm{L}, \mathrm{MCL}-1$ and A1) contain all four $\mathrm{BH}$ domains, of which the $\mathrm{BH} 1-\mathrm{BH} 3$ domains structurally form a pocket capable of binding the $\mathrm{BH} 3$ domains of other family members. Unlike the anti-apoptotic members, pro-apoptotic members can be subdivided by the number of BH domains they possess. The more conserved multidomain pro-apoptotic members (BAX, BAK, and $\mathrm{BOK}$ ) possess the $\mathrm{BH} 1, \mathrm{BH} 2$ and $\mathrm{BH} 3$ domains, which have been also shown to form a pocket by NMR structural analysis. In contrast, the BH3-only members (BID, BAD, BIM, BIK, NOXA and PUMA) contain only the BH3 minimal death domain and do not show sequence conservation outside this domain. Reprinted with permission from Macmillan Publishers Ltd: Opferman JT, Korsmeyer SJ. 2003. Apoptosis in the development and maintenance of the immune system. Nature Immunology 4: 410-415. 


\section{Myeloid Cell Leukemia-1}

\section{Anti-apoptotic MCL-1 and Cell Survival}

MCL-1 is a pro-survival BCL-2 family member that was previously cloned as an early response gene up-regulated during TPA-induced differentiation of ML-1 human myeloid leukemia cell line [8]. Sequence comparison of mammalian anti-apoptotic BCL2 family revealed that C-terminus of MCL-1 shares significant homology with BCL-2 and $\mathrm{BCL}-\mathrm{X}_{\mathrm{L}}$ and contains three $\mathrm{BH}$ domains $(\mathrm{BH} 2, \mathrm{BH} 1$ and $\mathrm{BH} 3)$ that form a hydrophobic cleft to which a $\mathrm{BH} 3$ amphipathic alpha-helix of other pro-apoptotic members can bind [9]. However, MCL-1's N-terminus is mostly unstructured and contains signal sequence like characteristics, like PEST sequences (enriched in proline, glutamic acid, serine, and threonine), accompanied by four pairs of arginines [8]. These prominent features are also present in various oncoproteins and other proteins which undergo rapid turnover [8]. Significantly, studies showed that $\mathrm{BH}$ domains were not disrupted by a $151 \mathrm{~N}$-terminal or $23 \mathrm{C}$-terminal amino acids truncation because the truncated mutant retains the ability to interact with BIM [10]. Therefore, neither the Nterminus nor C-terminus of MCL-1 is required for its anti-apoptotic function.

MCL-1's non-redundant and distinct role, compared with other pro-survival BCL2 family members, has been uncovered by gene-ablation methods. Bcl-2 knockout mice were viable at birth but displayed growth retardation and early postnatal mortality with severe lymphoid apoptosis, polycystic kidneys, and hypopigmentated hair [11]. Bcl- $X_{L}$ deficient mice died around embryonic day (E) 13 with dramatic apoptosis of immature hematopoietic cells and neurons [12]. Bcl-w and $A 1$ knockout mice have also been proved viable but manifested as spermatogenesis defects and minor defects in hematopoiesis, respectively [13-15]. Significantly, deletion of $\mathrm{Mcl}-1$ resulted in embryonic lethality due to peri-implantation failure around E3.5 [16].

Induced deletion of $\mathrm{Mcl}-1$ during early stage of lymphocyte differentiation leads to dramatically increased apoptosis and arrested the lymphocyte development at pre-Bcell and double-negative T-cell stages. Similar studies also showed MCL-1 is required for the survival of T cells at multiple stages $[17,18]$. In addition, $M c l-1$-induced deletion in early hematopoietic cells results in severe anemia and profoundly reduced bone marrow cells including hematopoietic stem cells (HSCs), common myeloid progenitors (CMPs), and common lymphoid progenitors (CLPs) [19] (Figure 1-3). MCL-1 also plays a selective role during myelopoiesis, supported by the observation that lineage-specific deletion of $\mathrm{Mcl}-1$ in myeloid precursors resulted in loss of mature neutrophils, but not macrophages or monocytes [20]. However, although MCL-1 is dispensable for the survival and differentiation of mature macrophages, it plays a necessary role in regulating macrophage effector responses during bacterial phagocytosis [21]. Moreover, studies also identified the absolute requirement for MCL-1 in the sustained survival of NK cells in vivo under the direct regulation of IL-15 and the survival as well as maintenance of plasma cells $[22,23]$. In contrast, loss of BCL-2 and BCL-X $\mathrm{X}_{\mathrm{L}}$ leads to defects of mature 


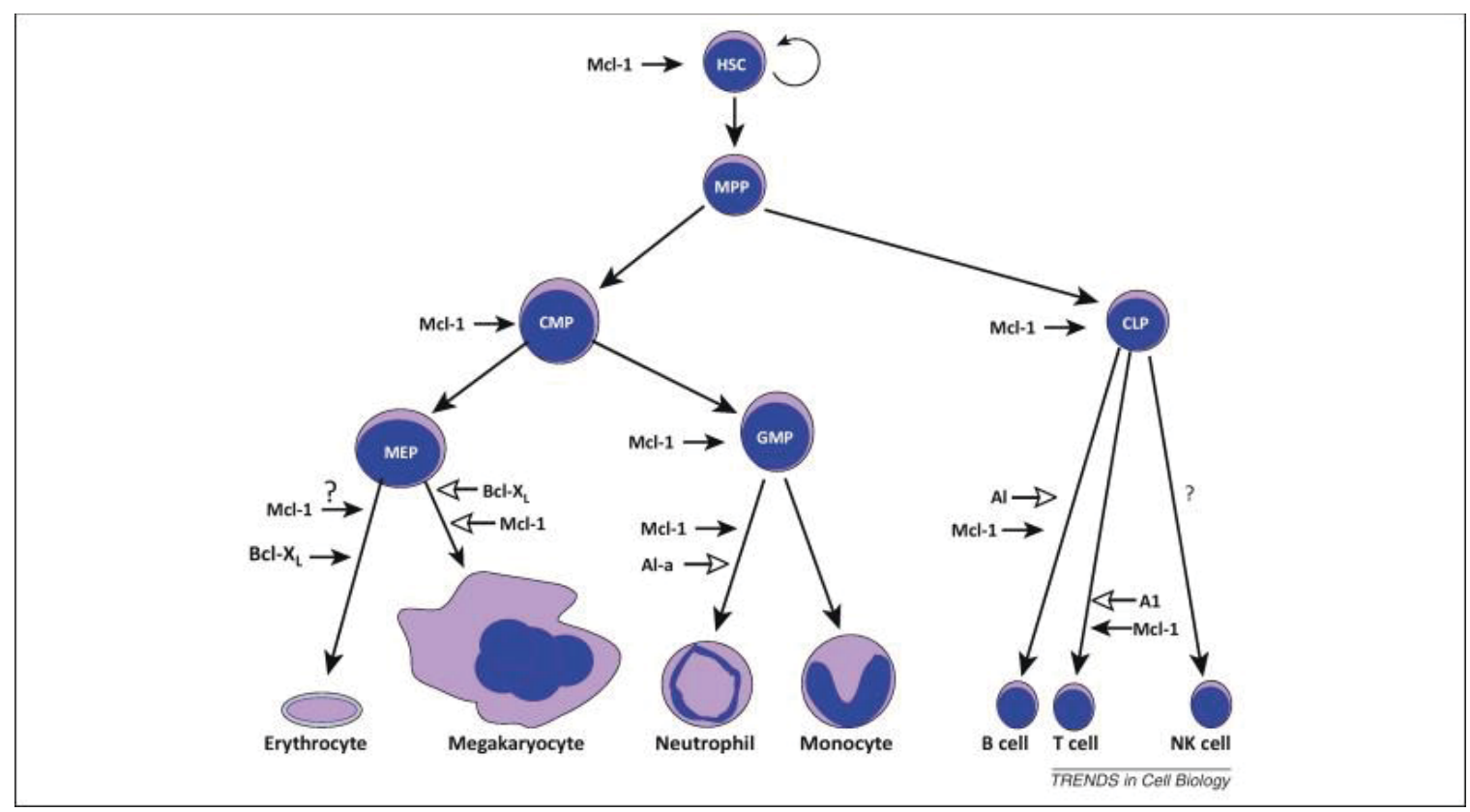

Figure 1-3. Role of anti-apoptotic regulators during hematopoiesis

All blood cell lineages arise from a hematopoietic stem cell (HSC) that is capable of selfrenewal and has an indefinite lifespan. HSCs give rise to multipotent progenitors (MPPs) that retain the ability to give rise to all blood cell lineages, but lack long-term selfrenewal capacity. MPPs can produce two progenitors, common myeloid progenitors (CMPs) and common lymphoid progenitors (CLPs). CLPs can produce the lymphoid lineages (B, T, and perhaps NK cells). CMPs produce at least two other oligopotent progenitor populations, the megakaryocyte erythroid progenitor (MEP) that produce red blood cells (erythrocytes) and megakaryocytes (generate platelets) and the granulocyte monocyte progenitor (GMP) that produces granulocytes (neutrophils) and monocytes (macrophages). Listed beside each differentiation step or progenitor population are the known anti-apoptotic regulators that promote the survival of the given population. Antiapoptotic MCL-1 has multiple checkpoints, because it has been illustrated to be critical for the survival of several multipotent and oligopotent progenitor populations (HSC, CMP, CLP, and GMP) and for the differentiation of granulocytes, but interestingly not the monocyte lineage. A1-a, a murine ortholog of BFL-1, and other A1 isoforms have also been shown to play an important role in promoting neutrophil survival in response to stress but are not absolutely required for development, primarily effecting mature cell survival (indicated by open arrowhead). A1 isoforms also play a role, but are not essential in lymphoid development. In the erythroid lineage, it appears that anti-apoptotic BCL- $\mathrm{X}_{\mathrm{L}}$ is the essential survival molecule, but unpublished data indicate that MCL-1 may also play an essential role during early differentiation. In the megakaryocytic lineage, it appears that, although neither MCL-1 nor BCL-xL is solely responsible for survival, the two pro-survival molecules appear to have overlapping functions (indicated by open arrowheads) in promoting megakaryocyte survival. Reprinted with permission from Elsevier Ltd: Perciavalle RM, Opferman JT. 2013. Delving deeper: MCL-1's contributions to normal and cancer biology. Trends in Cell Biology 23: 22-29. 
lymphocytes and red blood cells, respectively [11,24]. A1 isoforms play a role, but are not indispensable in granulocyte and lymphoid development [14,25]. These studies indicate MCL-1 is essential for the survival and differentiation of multiple hematopoietic lineages while the other pro-survival members play much more selective roles in hematopoiesis (Figure 1-3).

In addition to its essential role at different stages of hematopoiesis, MCL-1 is an important pro-survival factor in the hearts supported by the evidences that cardiacspecific ablation of $\mathrm{Mcl}-1$ resulted in a rapidly fatal, dilated cardiomyopathy by a loss of cardiac contractility, abnormal mitochondria ultrastructure, and defective mitochondrial respiration [26, 27]. MCL-1 is also a required molecule for cortical neurogenesis and the survival of neurons under DNA damage. Induced deletion of $\mathrm{Mcl}-1$ resulted in widespread cell death of neuronal progenitors and predisposed neurons to acute DNA damage [28]. Moreover, MCL-1 has been reported to be expressed in many normal human tissues, such as various epithelial tissues, neuroendocrine cells, sympathetic neurons, pancreatic islets cells, Leydig cells of the testis, cardiac muscles, skeletal muscles, chondrocytes, and hepatocytes [29,30]. The function of MCL-1 in many of those human tissues still remains to be established.

MCL-1 is also one of the most frequently amplified genes in a variety of human cancers, such as acute lymphoblastic leukemia (ALL), acute myelogenous leukemia (AML), chronic lymphocytic leukemia (CLL), chronic myelogenous leukemia (CML), neuroblastoma, melanoma, breast, lung cancer, etc. [31]. More importantly, elevated level of MCL-1 expression often correlates with disease progression, chemotherapeutic resistance and relapse [32-35]. The rationale for cancer cells displaying high level of MCL-1 is that they often violate important cellular checkpoints which can drive the cells to die through apoptosis. Thus, cancer cells need certain strategies for survival under the apoptotic stress by either downregulating pro-apoptotic regulators or, more frequently, upregulating pro-survival members like MCL-1 [36]. Kotschy et al. developed a small molecule S63845 that specifically binds to the BH3-binding groove of MCL-1 with high affinity and showed its potent anti-tumor activity with an acceptable safety margin either alone or in combination with other anti-cancer drugs in treating a wide range of tumors, including multiple myeloma, leukemia, lymphoma cells and various solid tumors [37].

\section{Non-canonical Function of MCL-1}

Sub-mitochondrial Localization of MCL-1. MCL-1 protein usually appears as a doublet ( $40 \mathrm{kD}$ and $36 \mathrm{kD}$ ) or even triplet $(40 \mathrm{kD}, 38 \mathrm{kD}$ and $36 \mathrm{kD})$ on immunoblots of many cell types. Several approaches have been adopted to decipher the banding pattern [38]. Phosphorylation of MCL-1 has been proposed to give rise to the $40 \mathrm{kD}$ isoform, however, phosphatase treatment does not abolish the $40 \mathrm{kD}$ species. It has been suggested that non-canonical translational initiation produces the faster-migrating $36 \mathrm{kD}$ protein, however, mutation of the start codon of MCL-1 only generates a $30 \mathrm{kD}$ truncated protein from a downstream ATG codon. Furthermore, ablation of the putative splice donor and 
acceptor still gives rise to the same banding pattern indicating that a non-canonical messenger RNA splicing is not the reason [38]. Therefore, proteolytic cleavage is the most likely contributor to the pattern. Indeed, Edman sequencing reveals two cleavage sites, one between isoleucine 10 and glycine 11 and another between leucine 33 and valine 34. Consequently, proteolytic cleavage of the N terminus of MCL-1 generates three different MCL-1 species: full-length $40 \mathrm{kD}$, middle band $38 \mathrm{kD}$ cleaved at isoleucine 10 and lower band $36 \mathrm{kD}$ cleaved at leucine 33 [38].

Unexpectedly, further experiments exhibited different mitochondrial sublocalization; the upper and middle forms of MCL-1 (40 kD and $38 \mathrm{kD})$ reside in the outer mitochondrial membrane (OMM) as both species are sensitive to proteinase $\mathrm{K}$ digestion performed on the isolated mitochondria, whereas the lower form $(36 \mathrm{kD})$ appears to be confined within the mitochondrial matrix as it is completely resistant to enzyme digestion. Moreover, the importation of MCL-1 into the mitochondria is mediated by the translocases of outer membrane (TOM) and inner membrane (TIM) supported by the evidence that RNA-mediated interference (RNAi) gene silencing of either TOM40 or TIM50 abrogates its import, rendering it completely proteinase $\mathrm{K}$ sensitive [38]. A similar study also showed that the fast-mobility (FM) isoform of MCL-1 is localized to the mitochondrial matrix and that a mitochondrial processing peptidase (MPP) is responsible for the N-terminal processing of MCL-1 to generate the FM species. The Nterminal 33 amino acids of mouse MCL-1 serve both as mitochondrial targeting and processing signal [39].

MCL-1 Possesses Multiple Functions in Mitochondria. Perciavalle et al. further have successfully made mutants to dissect MCL-1's two isoforms and shown that two species of MCL-1 retain different functions due to distinct subcellular localization. The OMM localized MCL-1 performs the classical anti-apoptotic function by antagonizing BAX and BAK. However, the matrix localized MCL-1 does not possess anti-apoptotic activity, but plays a role in maintaining mitochondrial ultrastructure, promoting mitochondrial fusion and fission, supporting the assembly of ATP synthase oligomers and oxidative phosphorylation. As supported by the SV40-transformed Mcl$1^{\text {flox/flox }}$ Rosa-ERCreT2 MEFs, when deletion of $\mathrm{Mcl}-1$ is induced by tamoxifen, it results in abnormal mitochondrial cristae structure, delayed mitochondrial fusion and fission, decreased ATP levels, mitochondrial membrane potential, oxygen consumption rates (OCRs), mitochondrial DNA contents, increased reactive oxygen species (ROS), and defected electron transport chain (ETC) supercomplexes and ATP synthase oligomerization. These phenotypes could be only rescued by full-length or matrix localized species of MCL-1 but not by the OMM localized isoform [38]. Therefore, other than its classical anti-apoptotic function, MCL-1 performs multiple functions in mitochondria in promoting mitochondrial physiology and supporting mitochondrial ultrastructure [40] (Figure 1-4). However, whether MCL-1 functions directly or indirectly to facilitate mitochondrial physiology still requires further investigation. 
(a)

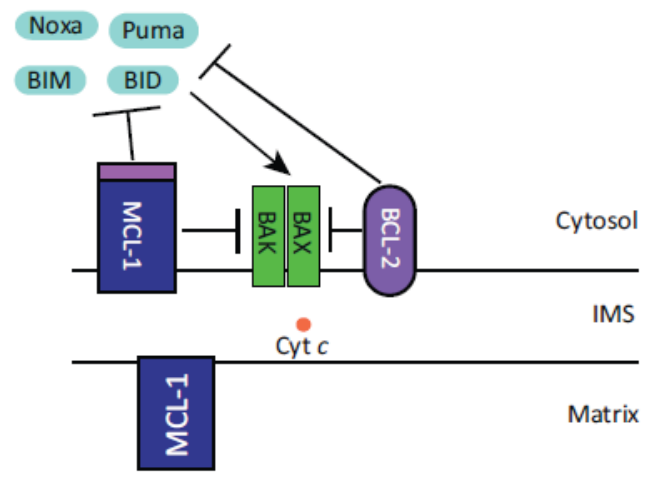

(b)

"Role in mitochondrial physiology"
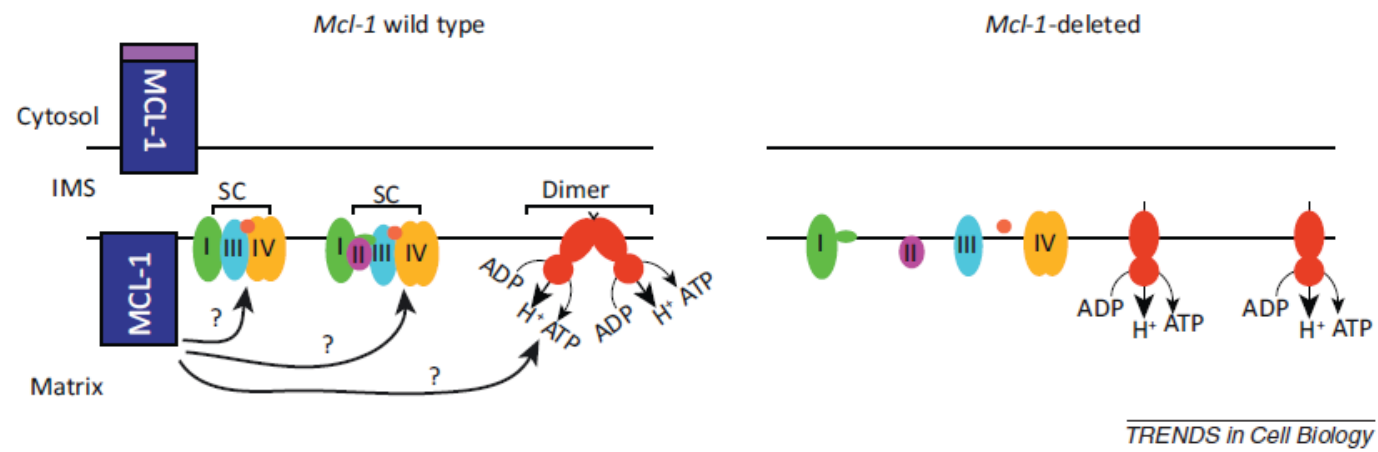

Figure 1-4. Model for MCL-1's potential functions in mitochondria MCL-1 possesses multiple functions in mitochondria. (a) On the outer mitochondrial membrane (OMM), MCL-1 functions like other anti-apoptotic BCL-2 family members where it acts to prevent the activation of BAX and BAK to prevent cell death. MCL-1 can directly bind BH3-only family members, such as BIM, sequestering them away from the pro-apoptotic effectors BAX and BAK. Alternatively, MCL-1 may directly bind BAX and BAK and maintain them in an inactive conformation. (b) During mitochondrial importation, the full-length MCL-1 is proteolytically truncated at its amino terminus. The truncated, matrix-localized MCL-1 resides within the inner mitochondrial membrane (IMM), where it functions to maintain mitochondrial cristae ultrastructure and promotes the assembly of the electron transport chain (ETC) complexes into higher-order assemblies known as supercomplexes. The assembly into supercomplexes has been shown to facilitate electron transport efficiency and reduce the production of deleterious reactive oxygen species. Additionally, matrix-localized MCL-1 facilitates the higher order assembly of the ATP synthase complexes into dimers and oligomers. Proper assembly of oligomeric ATP synthase has been implicated as being an important determinant of inner membrane cristae structure. Genetic ablation of $\mathrm{Mcl}-1$ results in defects in both supercompexes and ATP synthase oligomer assembly. Whether MCL-1 acts directly or indirectly to facilitate these macromolecular assemblies of the electron transport supercomplexes or ATP synthase oligomers remains unclear. Reprinted with permission from Elsevier Ltd: Perciavalle RM, Opferman JT. 2013. Delving deeper: MCL-1's contributions to normal and cancer biology. Trends in cell biology 23: 22-29. 


\section{Mitochondria in Human Development and Diseases}

Mitochondria are cytosolic double-membrane bound organelles found in almost all eukaryotic organisms and are known to be essential for cellular life and death regulation. They play a critical role in the life of the cell by carrying out energy-yielding oxidative reactions and electron transport chain activities which provide vast majority of ATP necessary for all cellular functions. Interruption of this mitochondrial function in vivo results in death, as dramatically presented by the mitochondrial electron transport chain (ETC) poisons as cyanide [41]. The proteins involved in oxidative phosphorylation (OXPHOS) and ETC components reside within the mitochondrial inner membrane. The ETC utilizes hydrogens generated through either the tricarboxylic acid (TCA) cycle from carbohydrates or the fatty acid $\beta$-oxidation from fats and atomic oxygen to make ATP and water [42, 43] (Figure 1-5). Moreover, OXPHOS also generates the major endogenous source of reactive oxygen species (ROS) as byproducts. Mitochondrial ROS are essential signaling pathway molecules as well as potent mitogens, however, too much ROS is toxic to the cells and can result in cellular death. Mitochondrial ROS production and redox balance are modulated by the mitochondrial inner membrane electrochemical gradient [42, 43] (Figure 1-5).

\section{Mitochondria and Human Development}

Mitochondria are dynamic organelles, the shape, size and number of which are highly variable and are controlled by fusion and fission [44]. The core mitochondrial fusion machinery in mammals consists of two related proteins mitofusins MFN1 and MFN2, which are essential for adjacent mitochondria during the fusion process [45-49]. Optic atrophy protein 1 (OPA-1), a mitochondrial dynamin-related protein, is another required molecule for fusion of the mitochondrial inner membranes, a function that consistent with its intermembrane space localization and inner membrane association [50, 51]. Mitochondrial fission is dependent on the recruitment of a dynamin-related protein 1 (DRP1) in mammals from the cytosol. DRP1 has been proposed to assemble into rings and spirals that encircle and constrict the mitochondrial tubule during fission [52].

Mitochondrial dynamics has been well known for several important biological functions. First, the balance between fusion and fission controls the shape, length, and number of mitochondria, which is highly dependent on the cell type and cellular energy status [53]. Second, fusion and fission processes allow mitochondria to exchange lipid membranes, mtDNAs and other intra-mitochondrial content, which helps maintain a healthy mitochondrial population $[49,54,55]$. Third, the dynamic characteristics of mitochondria help to distribute the mitochondria to specific subcellular compartment, which is extremely important in highly polarized cells, like neurons [56-58]. Due to these essential cellular functions, mitochondria are required for various biological processes, especially human development, and perturbations result in specific developmental defects. 
Figure 1-5. Mitochondrial physiology

Mitochondria lie at the nexus of most biosynthetic pathways, produce much of the cellular energy through oxidative phosphorylation (OXPHOS), regulate mitochondrial and cellular redox status, generate most of the reactive oxygen species (ROS), regulate $\mathrm{Ca}^{2+}$ concentrations and can initiate apoptosis by the activation of the mitochondrial permeability transition pore (mtPTP). The mtPTP can be activated by a decreased membrane potential, high-energy phosphates (such as ADP), a more-oxidized redox status, and/or increased mitochondrial matrix $\mathrm{Ca}^{2+}$ and ROS concentrations. Reducing equivalents and acetyl-CoA enter the mitochondrion via pyruvate and fatty acids. Pyruvate is transported through the mitochondrial inner membrane by the pyruvate transporter (PT), binds to pyruvate dehydrogenase (PDH), which may be membraneassociated, and is oxidatively decarboxylated to produce acetyl-CoA. Inhibition of mitochondrial function results in pyruvate accumulation in the cytosol, where it can be reduced to lactate. Fatty acids are imported into the mitochondrion bound to carnitine. In the cytosol, fatty acids bound to CoA are transferred to carnitine, transported through the outer and inner mitochondrial membranes, and then transferred back to CoA for $\beta$ oxidation. The transfer of fatty acyl groups between CoA and carnitine is mediated by the carnitine palmitoyltransferases (not shown). As a by-product of OXPHOS - the substrates and products of which are transported through the outer membrane by the voltage-dependent anion channels (VDACs) - mitochondria generate ROS by the donation of excess electrons from complexes I and III directly to $\mathrm{O}_{2}$ to generate superoxide anions $\left(\mathrm{O}_{2}{ }^{--}\right)$. Matrix $\mathrm{O}_{2}{ }^{--}$, primarily from complex $\mathrm{I}$, is dismutated to $\mathrm{H}_{2} \mathrm{O}_{2}$ by the mitochondrial matrix Mn superoxide dismutase (MnSOD; also known as SOD2), while intermembrane-space $\mathrm{O}_{2}{ }^{--}$, which is primarily from complex III, is dismutated by $\mathrm{Cu} / \mathrm{Zn}$ superoxide dismutase $\left(\mathrm{Cu} / \mathrm{ZnSOD}\right.$; also known as SOD1). $\mathrm{H}_{2} \mathrm{O}_{2}$ can be reduced to water by glutathione peroxidase using reduced glutathione as an electrondonor. Oxidized glutathione is reduced by glutathione reductase using NADPH as a reductant. In the presence of reduced transition metals, $\mathrm{H}_{2} \mathrm{O}_{2}$ can be reduced to hydroxyl radicals $(\bullet \mathrm{OH})$, which are the most reactive ROS. The mtPTP is a protein complex that is thought to include the translocator protein (TSPO; also known as PBR), an unknown innermembrane channel, adenine nucleotide translocators (ANTs) and the cyclosporine-Asensitive cyclophilin D (CYPD; also known as PPID), which are regulatory, in association with the BCL-2 pro- and anti-apoptotic family members. When activated, the mtPTP forms a channel between the inner and outer membranes, which short-circuits $\Delta \mathrm{P}$. This is associated with the aggregation of BAX and BAD in the mitochondrial outer membrane to form a megachannel. The megachannel releases pro-apoptotic proteins from the intermembrane space into the cytosol to initiate the degradation of the cellular proteins and DNA. AIF, apoptosis-inducing factor; CoA-SH, coenzyme A with a free sulphydryl group; CoQ, coenzyme Q10; ENDOG, mitochondrial endonuclease G; GPX, glutathione peroxidase; LDH, lactate dehydrogenase; OAA, oxaloacetate; SMAC, second mitochondria-derived activator of caspase; TCA, tricarboxylic acid. Reprinted with permission from Macmillan Publishers Ltd: Wallace DC. 2012. Mitochondria and cancer. Nature Reviews Cancer 12: 685-698. 


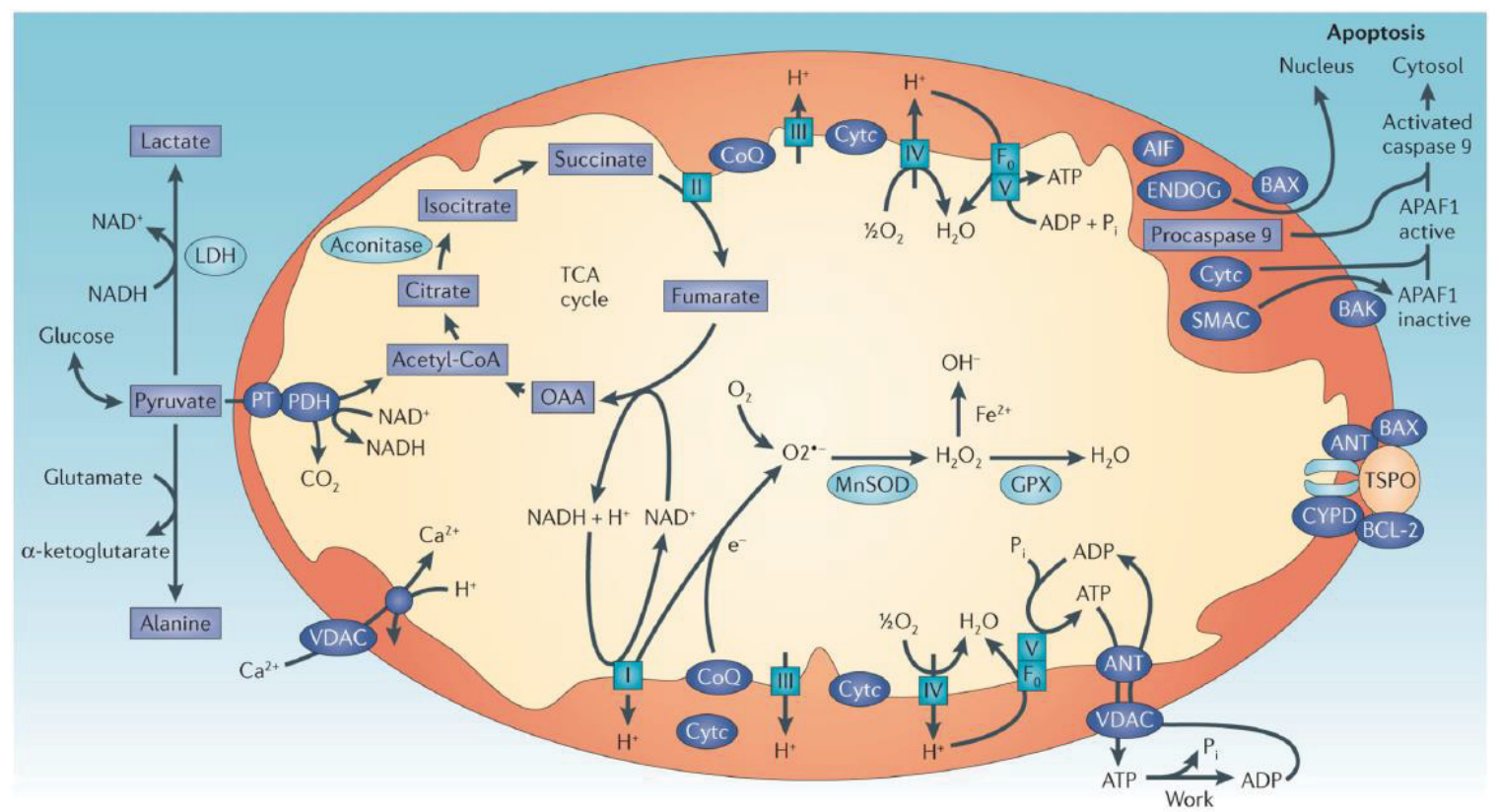


Previous studies revealed that mice deficient in either MFN1, MFN2 or OPA-1 die in midgestation [54, 59-61]. They further showed that MFN2 lacking embryos have a severe and specific disruption of the placental trophoblast giant cell layer. However, MFN1-deficient giant cell layers are normal but MFN1 deficient mice still die due to placental defects $[54,62]$. A case of a newborn girl bearing a dominant-negative dynamin-related protein 1 (DRP1) allele has been reported. The patient died around 1 month old and had a wide range of defects, such as microcephaly, abnormal brain development, optic atrophy and hypoplasia, persistent lactic academia, and elevated serum very-long-chain fatty acid [63].

Human reproduction, from oogenesis, fertilization to peri-implantation embryo development, has been suggested to be highly associated with the rate of mitochondrial replication as well as their activities. The optimal rate of oxygen consumption and fine regulation of aerobic and anaerobic respiration pathways are required for proper development [64].

\section{Mitochondria and Human Diseases}

Mitochondria are more than just a powerhouse of the cell. They have been reported to be intensively involved in the regulation of metabolism, cell-cycle control, development, antiviral responses and cell death (discussed earlier) [65]. Mitochondrion distinguishes itself from other organelles because it has its own mitochondrial DNA (mtDNA). The modern mammalian mtDNA has 13 polypeptide genes which encode essential components of OXPHOS. It also encodes the 12S and 16S rRNA genes and the 22 tRNA genes required for mitochondrial protein synthesis [66]. Nuclear genes encode the remaining mitochondrial OXPHOS proteins, the metabolic enzymes, the DNA and RNA polymerases, the ribosomal components and the mtDNA regulatory factors [42]. Mutations on either mtDNA or mitochondrial function associated nuclear genes have been reported to be related to human diseases [42].

Human Diseases Associated with MtDNA Mutations. Mutations from either base substitutions or rearrangements of mtDNA causes human diseases. A missense mutation from a $\mathrm{G}$ to A transition in mitochondrial ND6 gene, which encodes a subunit of NADH dehydrogenase, has been associated with Leber's hereditary optic neuropathy (LHON) [67]. Patients with LHON present with sudden-onset blindness in their early twenties because of the death of the optic nerve [67, 68]. Rearrangement mutations of mtDNA lead to diseases including chronic progressive external ophthalmoplegia (CPEO), Kearns-Sayre Syndrome (KSS), diabetes mellitus inherited from maternal side and deafness [69-72]. CPEO and KSS patients, for example, present with similar mitochondrial myopathy including ophthalmoplegia, ptosis and a subset of cardiac, renal, endocrine problems. 


\section{Human Diseases from Mutations in Mitochondrial Function Related Nuclear}

Genes. Mitochondrial diseases resulting from mutations in mitochondrial functions related nuclear genes share many of the clinical characteristics of mtDNA mutations. For instance, a mutation of the gene encoding one structural protein of electron transport chain (ETC) complex I has been reported in a young patient presenting hypotonia, mental retardation, seizure episodes, and basal ganglia degeneration [73]. Friedreich's ataxia, an autosomal recessive disease, is an example that mutations in nuclear genes can affect OXPHOS or destabilize mtDNA indirectly. The encoded protein, frataxin, is imported to the mitochondrial inner membrane and performs function to transport iron out of the mitochondrion. When mutated, iron accumulates in the mitochondrial matrix, stimulating the overproduction of oxidative stresses. Patients present with cerebellar ataxia, peripheral neuropathy, and hypertrophic cardiomyopathy [74].

\section{Mitochondria and Cancer}

Functional mitochondria are essential for cancer cell survival. Contrary to conventional opinions, although mutations affecting mitochondrial functions are common in cancer cells, they do not inactivate the energy and metabolic reactions but rather reprogram the mitochondrial bioenergetics and biosynthetic status to meet the requirements of the cancer cells [43]. And cancer therapies targeting mitochondria have been shown to be promising for cancer treatment [75].

MtDNA Mutations and Cancer. Although functional mitochondria are essential for cancer cell survival, mtDNA mutations and/or reduced mtDNA copy numbers are common features of cancer cells, which suggests that altered mitochondrial bioenergetics and/or metabolism play an important role in tumor transformation and maintenance [43]. For example, the missense mutation of MTND3 gene, encodes ETC complex I subunit, has been reported to be linked with increased risk for breast cancer in African American women [76]. And another missense mutation of cytochrome $c$ oxidase subunit 1 (MTCO1) T6777C has been associated with epithelial ovarian cancer, along with several nuclear DNA mitochondrial genes variants [77]. A meta-analysis has revealed that many cancer-associated mtDNA mutations consistently and obviously inhibit OXPHOS, which might serve to either stimulate tumor transformation or promote cancer cell adaptation to the tumor environment [43].

Mitochondrial Signaling Pathway and Cancer. Several hallmark cancer signaling pathways have been reported to reprogram mitochondrial physiology in order to promote tumor cell transformation, including c-MYC, K-RAS, PI3K and TP53 signaling pathways. For example, in addition to the various effects of oncogenic K-RAS signaling on proliferation, apoptosis and metabolism, it has also been reported to downregulate mitochondrial respiration and promote mitochondrial fission to support malignant transformation [78]. Another classical example should be PI3K-AKT-mTOR signaling pathway which has been shown to be overactive in many human cancers. The pathway in 
cancer cells is often overstimulated by either oncogenic mutations in signaling kinases or loss-of-function mutations of tumor suppressor proteins like PTEN, which then activates the downstream effector mechanistic target of rapamycin (mTOR). mTOR, a core component of two distinct protein complexes mTORC1 and mTORC2, functions as a serine/ threonine protein kinase that regulates many biological activities, such as cell growth, proliferation, cell survival, protein synthesis, autophagy, and transcription [79]. PI3K-AKT-mTOR signaling pathway also inhibits carnitine palmitoyltransferase IA which is the rate limiting step for fatty acid oxidation and phosphorylates hexokinase II which is known to capture mitochondrial ATP and utilize it to convert glucose to glucose-6-phosphate. All these effects from activation of PI3K-AKT-mTOR pathway help the shift from oxidative phosphorylation to more glycolytic metabolism, which is more favorable for cancer cell transformation and proliferation [43].

Oxidative Stress and Cancer. Reactive oxygen species (ROS), in the form of superoxide $\left(\mathrm{O}_{2} \cdot\right)$, hydroxyl free radicals $(\mathrm{OH})$, and hydrogen peroxide $\left(\mathrm{H}_{2} \mathrm{O}_{2}\right)$, are produced from multiple physiological reactions, including electron transport through the ETC complex 1 and NAD(P)H oxidases. Mitochondria not only are the major contributors to cellular ROS production but also have multiple antioxidant strategies to neutralize ROS, such as superoxide dismutase (SOD2), glutathione, thioredoxin, and peroxiredoxins [78]. There are two faces of ROS biology: redox signaling and oxidative stress [80]. While redox signaling is essential for various cellular processes, such as proper cellular differentiation, proliferation, and tissue regeneration, high levels of ROS leads to oxidative stress which incur damage to DNA, protein or lipids, resulting in genomic instability to promote cancer cell transformation [80].

Well-known tumor suppressor p53 controls the expression of many antioxidant genes and it is been shown that tumor initiation in $\operatorname{Trp} 53^{-/-}$mouse models can be successfully inhibited by antioxidant NAC supplementation in the diet, indicating a tumor suppressive function of p53 through regulating antioxidant and metabolism genes rather than apoptosis and cell cycle arrest [81-83]. Another example is that $\mathrm{H}_{2} \mathrm{O}_{2}$ has been suggested to inactivate tumor suppressor PTEN by oxidizing its cysteine residues and promoting the formation of a disulfide bond, which prevents PTEN from inhibiting PI3K pathway [84]. ROS-mediated regulation of oncogenic signaling has also been revealed to affect metastasis. As reported by Porporato et al. oxidation of cysteines in SRC increases its oncogenicity, facilitating tumor cell migration and metastasis in multiple tumor types, which can be blocked by a ROS scavenger [85].

While ROS play an essential role in activating proper mitogenic signals to promote cancer cell proliferation, survival and metabolic adaptation, too much ROS make cancer cells susceptible to oxidative stress-induced cell death. Therefore, cancer cells have to maintain ROS levels in a window which can stimulate proliferation without causing cytotoxicity [80]. The dependence on upregulated antioxidant pathways may act as an Achilles heel for cancer therapies. Combined therapy of glutathione and thioredoxin inhibitors has been shown to limit tumor growth in breast cancer models both in vitro and in vivo [78]. 
Targeting Mitochondria for Cancer Therapy. Cancer cells adopt an extensive metabolic reprogramming, which makes their mitochondria structurally and functionally different from their normal counterparts and renders them more susceptible to the mitochondrial targeted therapies [75]. Several selective mitochondria targeted strategies have already been shown to be promising on a variety of cancer models. Targeting mitochondrial permeability transition pore complex (MPTP) is one of the examples [8691]. Multiple compounds have been developed to act on the components of the MPTP to induce the outer mitochondrial membrane permeabilization and apoptosis. Another strategy is to design compounds that induce the overproduction of ROS or inhibit antioxidant systems. Motexafin gadolinium has been suggested to selectively accumulate in many types of cancers, exhibiting an overproduction of ROS and enhancing the in vivo response to radiotherapy on lung cancer as well as pediatric glioblastoma $[92,93]$. There are now sufficient evidences suggesting shifting the balance of the ratio between proapoptotic versus anti-apoptotic BCL-2 proteins primes cancer cells to die through MOMP-dependent apoptosis. One of the most well-recognized and best established example is BH3 mimetics, ABT-737, which specifically binds to BCL-2, BCL-X $\mathrm{L}$ and BCL-w and induces tumor cell death [94-97]. Last but not least, approaches reversing the hyperglycolytic state of cancer cells also have been shown to be successful in clinical trials. 2-deoxy-D-glucose (2DG), an inhibitor of glycolysis, has been revealed to significantly synergize with cisplatin on the cytotoxicity in treating human head and neck cancer cells [97].

\section{CRISPR-Cas9 System for Genomic Editing}

In recent years, the development of genome engineering technologies, the process of making targeted genome modification in their endogenous context, allow researchers to rapidly and efficiently recapitulate biological functions or disease phenotypes of genetic mutations or epigenetic variants in cellular or animal models. It enables the direct correction of genetic or epigenetic defects in somatic tissues for genetically encoded disorders [98]. Generally speaking, directed genomic modification is achieved by targeting a genome-editing nuclease to the specific sequences in the genome, which recognize and cut at the specific site to generate double-strand breaks (DSBs). Generated DSBs can be further repaired by one of the two cellular endogenous DNA repair mechanisms: non-homologous end joining (NHEJ) or homology-directed repair (HDR) when a repair donor template DNA provided. NHEJ is highly efficient however errorprone, which results in small insertions or deletions (indels) at the targeted locus and therefore leads to shift reading frame to generate nonfunctional proteins. By contrast, HDR-based gene repair can be used to create knockin sequences at targeted site when a donor template DNA containing homologous arms is delivered [99].

There are four major strategies that are currently performed to introduce DSBs in the genome: meganucleases, zinc-finger nucleases (ZFNs), transcription activator-like effector nucleases (TALENs), and the clustered regularly interspaced short palindromic repeat (CRISPR)-Cas system [100-103]. Meganucleases target various sequences and they are considered as the most specific strategy due to the recognition sequences are 
long enough to occur only with a very low chance. However, the requirement that DNA recognition and cleavage sites need to be linked closely limits the broad application of those nucleases [101]. ZFNs are generated via the fusion of a zinc-finger DNA binding domain with the cleavage domain called FokI nuclease that acts as a dimer [102]. TALENs are composed of the DNA binding domain called transcription activator-like effector (TALE) and a FokI nuclease [103]. All three, meganucleases, ZFNs, and TALENs, create DNA targeted sequence recognition specificity via protein-DNA interaction and require labor- or time-consuming protein engineering or assembly [99].

RNA-guided endonuclease Cas9 from the microbial adaptive immune system CRISPR has been developed in the recent 5 years and provides a highly sensitive and precise system for achieving targeted mutagenesis in eukaryotic cells with its ease of customization and high efficiency. The Cas9 nuclease is paired and targeted to genomic DNA by a guide RNA consisting of a 20 -nucleotide sequence and a scaffold with the guide sequence required to be directly upstream of a 5'-NGG adjacent motif called PAM motif [104] (Figure 1-6). Then, Cas9 endonuclease induces a double strand break around 3 bp upstream of the PAM motif. DSBs can be repaired by either NHEJ pathway, which results in random indels to make a gene knockout, or homologous recombination, which allows for high fidelity and precise editing when a repair template is provided [104] (Figure 1-7).

CRISPR-Cas9 system has enabled efficient and accelerated generation of transgenic models to allow for recapitulating genetic mutations found in patient populations, which facilitates the study of disease mechanisms and therapeutic targets $[98,105]$. Researchers have also applied this technology to engineer isogenic ES or iPS from patients with specific mutations and then induce those stem cells to differentiate and recapitulate the disease development models and screen for therapeutic targets [106]. Genome-wide loss-of-function screening can be performed by mass synthesis and delivery of guide RNA libraries to disrupt thousands of genomic elements in parallel to study candidate genes affecting phenotypes [107, 108]. In addition to a research tool that has been widely used, CRISPR-Cas9 system is also a potential strategy for therapeutic purpose. For example, some monogenic diseases such as cystic fibrosis, sickle-cell anemia, etc. have loss-of-function mutations due to small deletions or replacements that can be corrected by Cas9-induced gene repair $[98,106]$. Other than repairing underlying mutations, Cas9-mediated NHEJ repair can be utilized for introducing protective mutations, such as Cas-9-induced inactivation of the CCR5 receptor, a co-receptor required for HIV incorporation [109]. Despite many of the advantages, challenges need to be addressed, such as finding the efficient delivery systems, reducing the off-target effects, and monitoring the long-term safety issues [98]. 


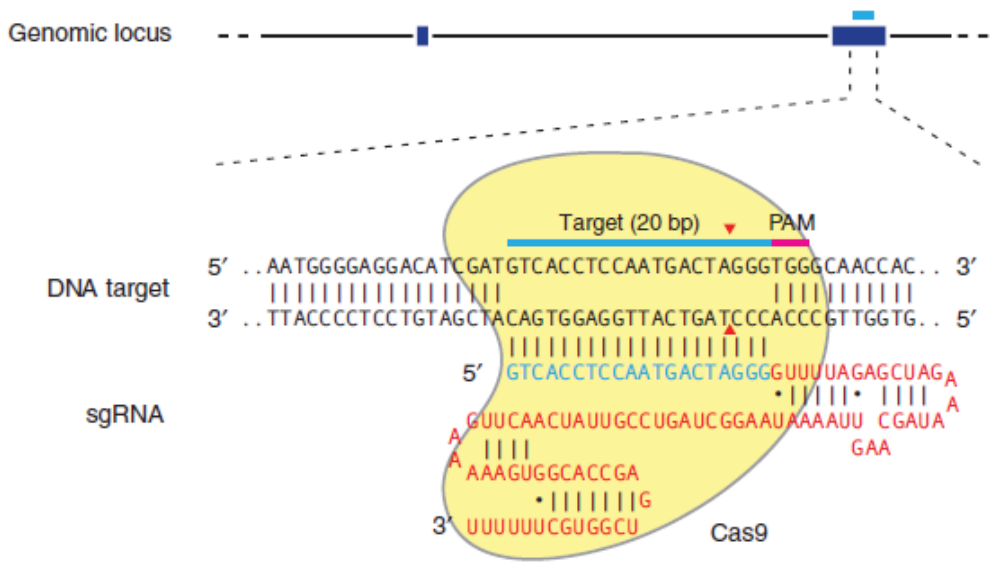

Figure 1-6. Schematic of the RNA-guided Cas9 nuclease

The Cas9 nuclease from $S$. pyogenes (in yellow) is targeted to genomic DNA (shown for example is the human EMXI locus) by a sgRNA consisting of a 20-nt guide sequence (blue) and a scaffold (red). The guide sequence pairs with the DNA target (blue bar on top strand), directly upstream of a requisite 5'-NGG adjacent motif (PAM; pink). Cas9 mediates a DSB around $3 \mathrm{bp}$ upstream of the PAM (red triangle). Reprinted with permission from Macmillan Publishers Ltd: Ran FA, Zhang F. 2013. Genome engineering using the CRISPR-Cas9 system. Nature Protocol 8: 2281-2308.

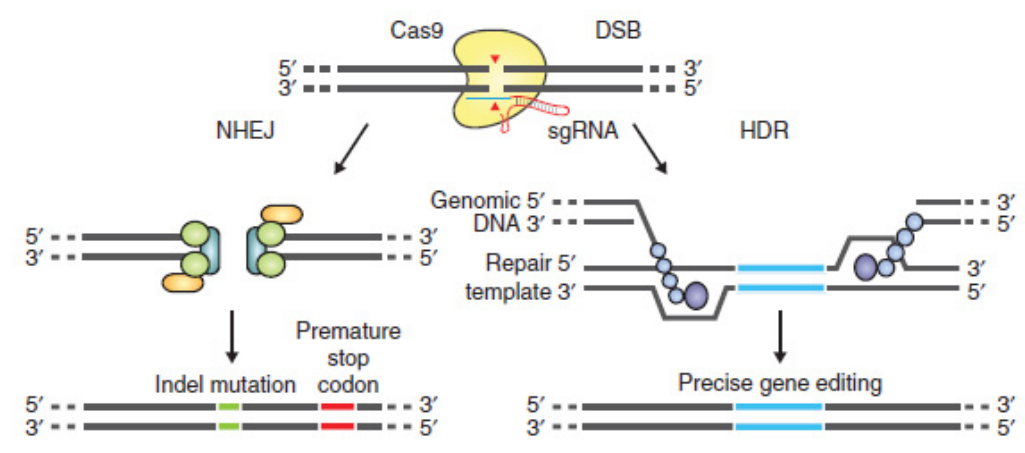

Figure 1-7. DSB repair promotes gene editing

DSBs induced by Cas9 (yellow) can be repaired in one of two ways: In the error-prone NHEJ pathway, the ends of a DSB are processed by endogenous DNA repair machinery and rejoined, which can result in random indel mutations at the site of junction. Indel mutations occurring within the coding region of a gene can result in frameshifts and the creation of a premature stop codon, resulting in gene knockout. Alternatively, a repair template in the form of a plasmid or single-stranded DNA oligonucleotides (ssODN) can be supplied to leverage the HDR pathway, which allows high fidelity and precise editing. Single-strand nicks to the DNA can also induce HDR. Reprinted with permission from Macmillan Publishers Ltd: Ran FA, Zhang F. 2013. Genome engineering using the CRISPR-Cas9 system. Nature Protocol 8: 2281-2308. 


\title{
CHAPTER 2. MATERIALS AND EXPERIMENTAL PROCEDURES
}

\author{
Mice \\ $M c l-1^{\text {flox/flox }} \mathrm{Cre}{ }^{\text {ERT2 }}, M c l-1^{\text {flox/WT }} \mathrm{Cre}{ }^{\text {ERT2 }}$ and $M c l-1^{\text {flox/flox }}$ ESCs were generated at
} E3.5 from the C57BL/6 mice with respective genotypes. Cre ${ }^{\text {ERT2 }}$ is a tamoxifen inducible Cre recombinase system. To induce deletion, five doses of tamoxifen emulsified in sunflower oil vehicle (Sigma) were performed through gavage (1 mg per dose; Sigma). All conditional mice were on a C57BL/6 background and littermates were used as controls. $\mathrm{Nu} / \mathrm{J}$ mice used for teratoma formation assays were obtained from Jackson Laboratory. C57BL/6J mice (Jackson Laboratory) were used for zygote injections. All mice were maintained in accordance with the Institutional Animal Care and Use Committee of St. Jude Children's Research Hospital as required by the U.S. Animal Welfare Act and National Institutes of Health Policy for proper care and use of laboratory animals for research.

\section{Cells and Cell Culture}

The 129/SvJ ES cell line F12, derived by Jacqueline Surtel (St. Jude Children's Research Hospital) was routinely cultured on tissue-culture treated dishes (Corning) in ESCs medium, consisting of 90\% glucose-free Dulbecco's modified Eagle's medium (DMEM, Lonza), 10\% fetal bovine serum (FBS, Hyclone), $100 \mathrm{U} / \mathrm{ml}$ penicillin, 100 $\mathrm{ug} / \mathrm{ml}$ streptomycin, $2 \mathrm{mM}$ L-glutamine (all from Invitrogen), $0.1 \mathrm{mM}$ mercaptoethanol (Gibco), and $10^{3} \mathrm{U} / \mathrm{ml}$ recombinant mouse leukemia inhibitory factor (LIF from Millipore). Mcl-1-conditional ESCs were derived at E3.5 from $\mathrm{Mcl}$-1-conditional mice breedings described previously. Once plugs were observed at E0.5, mice uteri were dissected under the microscope and blastocysts were flushed. Inner cell masses (ICMs) were separated from the whole blastocysts and transferred onto pre-coated irradiated MEFs. After expansion, single cell clones were picked for genotyping. Mcl-1 conditional ESCs were grown in the same medium as F12 ESCs.

SV40-transformed Mcl-1 conditional mouse embryonic fibroblasts (MEFs) have been previously described [17]. To induce $\mathrm{Mcl}-1$ deletion, the MEFs were treated for 48 hours with 100nM 4-hydroxy-tamoxifen (Sigma) in culture medium. HEK 293T cells were obtained from American Type Culture Collection. Arf ${ }^{\text {pull }}$ GFP MEFs, derived by Dr. Frederique Zindy (St. Jude Children's Research Hospital), were exposed to 4000 rads from a $\gamma$-radiation source and used as stromal cells. All MEFs, HEK 293T cells and irradiated MEFs were grown in 90\% glucose-free DMEM (Invitrogen) supplemented with 10\% FBS (Gibco), $100 \mathrm{U} / \mathrm{ml}$ penicillin, $100 \mathrm{ug} / \mathrm{ml}$ streptomycin, $2 \mathrm{mM}$ L-glutamine (all from Invitrogen), and $0.1 \mathrm{mM}$ mercaptoethanol (Gibco). 


\section{Plasmids, Expression Constructs, and Generation of Mutants}

All Mcl- 1 constructs were generated according to mouse $M c l-1$ cDNA. The mutants $M c l-1$ cleavage site (CS) 10,11 and $M c l-1$ CS 33, 34, in which isoleucine, lysine and leucine, valine were replaced by aspartic acid, were derived by site-directed mutagenesis (Stratagene). The mutant Mcl-1 Matrix was generated by fusing amino acids $1-58$ of $N$. crassa ATP-synthase to Mcl-1 N-terminal 34 amino acids truncated mutant. Mcl-1 ATG1 and M92 were derived by mutating the first start codon of Mcl-1 to TGA stop codon and $\mathrm{N}$-terminal 91 amino acid truncation, respectively.

\section{Ecotropic Retroviral Production and Cell Transduction}

Ecotropic retroviruses were produced by co-transfection of packaging plasmids (pMD-old-Gag-Pol and pCAG4-Eco) and retroviral expressing plasmids to HEK 293T cells by using FuGene6 (Roche Applied Bioscience). Collected viruses were filtered and applied on either ESCs or $\mathrm{Mcl}-1$ conditional MEFs together with $10 \mathrm{ug} / \mathrm{ml}$ polybrene (PB, AmericanBio).

\section{Crispr-Cas9 System for Genomic Targeting}

Nine guide RNAs were designed close to Mcl-1's genomic start codon and amino acid 10 isoleucine, 11 glycine and paired with the DNA target directly upstream to a 5'NGG as following:

CrRNA-F1, 5'- GTCCGACCATGTTTGGCCTG -3';
CrRNA-F2, 5'- GTTTGGCCTGCGGAGAAACG -3';
CrRNA-F3, 5'- GATGACTTGAACCTGTACTG -3';
CrRNA-F4, 5'- GCCAGCCTCGGCGCGGGCGG -3';
CrRNA-R1, 5'- GTCGGACGCCGCAGGCTGAG -3';
CrRNA-R2, 5'- GTTTCTCCGCAGGCCAAACA -3';
CrRNA-R3, 5'- ACCGCCGCCCGCGCCGAGGC -3';
CrRNA-2F, 5'- GCGGGCGGCGGTTCTCCGGC -3';
CrRNA-2R, 5'- GGCTGGCGCCGCCGCAGTAC -3'.

Then, each of the guide RNA was cloned into the all-in-one Cas9 (BB)-2A-GFP (Addgene plasmid ID: 48138) through BbsI enzyme site and validated by sequencing with a universal U6-forward primer:

\section{5'-GAGGGCCTATTTCCCATGATTCC-3'.}

Repair templates single-stranded DNA oligonucleotides (ssODNs) containing 5' and 3' homologous arms, amino acids 10 aspartic acid, 11 aspartic acid mutations as well as the mutated CRISPR-Cas9 binding sites were also designed by either symmetric or 
asymmetric strategies and synthesized through IDT (Integrated DNA Technologies) for making cleavage site amino acid 10,11 knock-in $\left(\mathrm{CS}^{10,11} \mathrm{KI}\right)$ mutagenesis as following:

Symmetric ssODN CS10,11 F3M, 5'- CCT GCG GCG TCC GAC CAT GTT TGG CCT GCG GAG AAA CGC GGT CGA TGA CCT CAA TCT ATA CTG CGG CGG CGC CAG CCT CGG CGC GGG CGG CGG TTC TCC G -3’;

Asymmetric ssODN CS10, 11 F3M, 5'- CCT CGC GCC GCG CCT TGG CCT CCT CGG CCA CCA GGC GCG CCC CTG CCG GAG AAC CGC CGC CCG CGC CGA GGC TGG CGC CGC CGC AGT ATA GAT TGA GGT CAT CGA CCG CGT TTC TCC GCA GGC CAA ACA TGG TCG GAC G -3';

Symmetric ssODN CS10,11 R2M, 5'- TTC CCC TCA GCC TGC GGC GTC CGA CCA TGT TTG GCC TGC GGC GCA ATG CGG TCG ATG ACT TGA ACC TGT ACT GCG GCG GCG CCA GCC TCG GCG CGG GCG G -3';

Asymmetric ssODN CS10, 11 R2M, 5'- TTC CGC CTG CCT CCG GTC TGG AGT CGC GGC CTT CCC CGC TCC TTC CCC TCA GCC TGC GGC GTC CGA CCA TGT TTG GCC TGC GGC GCA ATG CGG TCG ATG ACT TGA ACC TGT ACT GCG GCG GCG CCA GCC TCG GCG CG -3'.

Embryonic stem cells (ESCs) F12 were transfected with each guide RNA containing all-in-one plasmid as well as ssODNs repair template. 48 hours after transfection, ESCs were sorted for GFP positive cells. After sort, 20,000 cells were seeded in 10 centimeter $(\mathrm{cm})$ dish and growing as single cell clones. The leftover pool cells from each individual guide RNA were screened first by T7E1 surveyor nuclease digestion as well as by immunoblotting. After single cells grew out, they were screened by both Sanger sequencing and immunoblotting.

\section{Surveyor Nuclease Digestion}

DNA was extracted from ESCs for each guide RNA leftover pools. PCR was performed to amplify targeted $M c l-1$ amplicons by using the following primers:

Mcl-1 forward primer, 5'- AACGGCCTTCCTCACTCCTGACTT -3'; Mcl-1 reverse primer, 5'- AGTAGCGCGAGATGATCTCCAGCGA -3'.

Further the PCR amplified targeted sequences were denatured and re-hybridized. PCR reactions were subjected to endonuclease T7E1 digestion which would recognize the heteroduplexes formed by either wild-type and mutant sequences or two different mutant amplicons. The final PCR reactions treated in the absence or presence of T7E1 were evaluated by agarose gels.

\section{Immunoblotting, Co-immunoprecipitation, and Antibodies}

Cells or tissues were lysed in radioimmunoprecipitation assay (RIPA) buffer (50 $\mathrm{mM}$ Tris-HCL at $\mathrm{pH} 7.4,150 \mathrm{mM} \mathrm{NaCl}, 1 \%$ Triton X-100, $1 \mathrm{mM}$ EDTA, $1 \%$ sodium 
deoxycholate, $0.1 \%$ SDS) containing protease inhibitors (Cat: 11836145001, Roche) on ice for 30 minutes. Lysates were then collected by centrifugation and protein concentrations were measured by BCA assay (Pierce) with standard curve. 20 micrograms (ug) of each protein samples was loaded on 10\% NuPAGE Bis-Tris gels (Invitrogen) and MOPS SDS served as the trailing ion in the running buffer. For coimmunoprecipitation assays, whole cell lysates (WCLs) were collected and coupled with anti-BIM rat monoclonal antibody (Millipore) for one hour, and then precipitated with protein A/G-plus agarose (Santa Cruz Biotechnology). Immunocomplexes and postimmunoprecipitated supernatants were subjected to $10 \%$ NuPAGE Bis-Tris gels (Invitrogen), transferred to PVDF (Millipore) and developed by using Western Lightning ECL Pro (Perkin Elmer) on a LI-COR Odyssey (LI-COR, NB). The following antibodies were used: anti-MCL-1 (Rockland Immunochemical), anti-Actin (Millipore), anti-OPA-1 (BD Biosciences), anti-MnSOD (BD Biosciences), anti-BIM (BD Biosciences), antimBCL-2 (Clone 3F11, gift of S. Korsmeyer), anti-hBCL-2 (Clone 6C8, gift of S. Korsmeyer), anti-BAX (Millipore), anti-ATP5B (Santa Cruz Biotechnology), antiCaspase 3 (Cell Signaling Technology) and anti-PARP (Cell Signaling Technology).

\section{Genotyping and Sanger Sequencing}

Genomic DNA of single ES colonies or tail tissues of individual pups were extracted in $100 \mathrm{mM}$ Tris- $\mathrm{HCl}$ at $\mathrm{pH} 8.3,100 \mathrm{mM} \mathrm{NaCl}, 5 \mathrm{mM}$ EDTA, $1 \%$ Triton X-100, and $100 \mathrm{ug} / \mathrm{ml}$ proteinase K. Samples were incubated overnight at $55^{\circ} \mathrm{C}$ and $85^{\circ} \mathrm{C}$ for 45 minutes. Debris were removed by 13,000 rpm centrifugation for 10 minutes. Genomic DNA was amplified by using $M c l-1$ forward primer 5'- AACGGCCTTCCTCACTCC TGACTT -3' and $\mathrm{Mcl}-1$ reverse primer, 5'- AGTAGCGCGAGATGATCTCCAGCGA 3'. PCR products were purified (Qiagen PCR purification kit) and subjected to Sanger sequencing by using the same $\mathrm{Mcl}-1$ forward primer. Homozygous mutations were determined by comparison to mouse $\mathrm{Mcl}-1$ genomic sequences and heterozygous mutations were determined by filtering out the known sequence of the wild-type allele.

\section{Mass Spectrometry Identification}

MCL-1 wild-type (WT) and mutant $30 \mathrm{kD} \mathrm{ESCs}$ around 5 million each were washed with PBS and re-suspended in $0.5 \mathrm{ml}$ hypotonic buffer $(20 \mathrm{mM}$ Tris $\mathrm{HCl}$ at $\mathrm{pH}$ $7.4,10 \mathrm{mM} \mathrm{MgCl}_{2}$ containing protease inhibitors described previously), and then homogenized by brief sonication. After short centrifugation at $800 \mathrm{~g}$, the supernatant was taken for ultracentrifuge at $180,000 \mathrm{~g}$ for $10 \mathrm{~min}$ at $4^{\circ} \mathrm{C}$. The pellets were dissolved in 50 ul 1\% Triton X-100 in PBS containing PIC and centrifuged at 20,000g for 3 minutes.

The supernatant was collected and separated by SDS-PAGE. The bands were cut from the predicted regions, and then digested by $12.5 \mathrm{ng} / \mathrm{ul}$ trypsin in $50 \mathrm{mM} \mathrm{NH}_{4} \mathrm{HCO}_{3}$ at $\mathrm{pH}$ 8.5. The final peptide samples were loaded to $\mathrm{C} 18$ column ( $75 \mathrm{uM}$ internal diameter, $10 \mathrm{~cm}$ long, 1.9 um resin beads), eluted at 15-40\% buffer B gradient (buffer A: 
$0.2 \%$ formic acid, $5 \%$ acetonitrile; buffer B: $0.2 \%$ formic acid, $95 \%$ acetonitrile) at 300 $\mathrm{nl} /$ min for 6 hours on LTQ Orbitrap Velos (Thermo Scientific).

\section{Embryoid Body Formation}

Embryoid bodies (EBs) were generated by separating the undifferentiated mouse ESCs and resuspending 300 cells into $50 \mathrm{ul}$ hanging drops. Two days later, aggregates were collected and transferred to ultralow attachment dishes with EB culture medium, consisting of 90\% DMEM (Lonza), 10\% FBS (Hyclone), $100 \mathrm{U} / \mathrm{ml}$ penicillin, $100 \mathrm{ug} / \mathrm{ml}$ streptomycin, $2 \mathrm{mM}$ L-glutamine (all from Invitrogen), and $0.1 \mathrm{mM}$ mercaptoethanol (Gibco). The medium was changed every other day. EBs were collected by either day 4 or day 10 for further analysis.

\section{Cryosectioning and Immunofluorescent Staining of Embryoid Bodies}

Collected embryoid bodies (EBs) were precipitated and mounted in tissue freezing medium (Triangle Biomed), then saved at $-80^{\circ} \mathrm{C}$. Cut 10 um thick tissue sections by using a cryostat (Microm HM 505) onto the microscope slides (Fisher Scientific). Sections were air dried for 30 minutes at room temperature (RT) and the EBs fixed on the glass cover slip in $4 \%$ paraformaldehyde (PFA) in PBS supplemented with $0.1 \%$ Triton $\mathrm{X}-100$ for 15 minutes at RT, followed by permeabilization with $0.1 \%$ Triton X-100 in PBS for 5 minutes at RT. Cells were blocked with $10 \%$ goat serum in PBS. All primary antibodies were diluted in 3\% goat serum in PBS added to slides and incubated overnight at $4{ }^{\circ} \mathrm{C}$. Cells were treated with fluorescently coupled secondary antibody diluted in $3 \%$ goat serum in PBS, goat anti-mouse or goat anti-rabbit (Life Technologies) and were incubated for 1 hour at RT. Glue containing DAPI (ProLong Gold Antifade Mountant, Thermo Fisher Scientific) was added onto the EBs and covered by a glass. The glue was allowed to dry and all images were captured by using microscopy (Zeiss Axioskop II Plus).

\section{RNA Isolation and Quantification}

RNA was extracted from ESCs with TRIzol (Ambion) and reverse transcribed by using SuperScript III first-strand synthesis kit (Invitrogen). Fast real-time PCR with fast SYBR green (Bio-Rad) detection was performed by using QuantStudio 7 Flex (Life Technologies). And the following primers were used:
mSox-2 forward: 5'- GGTTACCTCTTCCTCCCACTCCAG -3'; mSox-2 reverse: 5'- TCACATGTGCGACAGGGGCAG -3'; mOct-4 forward: 5'- ATGGCATACTGTGGACCTCA -3'; mOct-4 reverse: 5' - AGCAGCTTGGCAAACTGTTC -3'; mSox7 forward: 5'- GACACCTTGGATCAGCTAAGCC -3'; mSox7 reverse: 5'- CCTCCAGCTCTATGACACACTG-3'; 
mFgf-5 forward: 5'- AAAGTCAATGGCTCCCACGAA -3';

mFgf-5 reverse: 5'- GGCACTTGCATGGAGTTTTCC -3';

mmHox-4 forward: 5' - TGGCGGCACAAGCAGACGAAAG -3';

m $m$ Hox-4 reverse: 5'- GTGAGGTTCACCCGACGTGCGAGA -3';

mIhh forward: 5'- ACGTGCATTGCTCTGTCAAGT -3';

mIhh reverse: 5'- CTGGAAAGCTCTCAGCCGGTT -3';

mGapdh forward: 5'- TGTGTCCGTCGTGGATCTGA -3';

m Gapdh reverse: 5'- CCTGCTTCACCACCTTCTTGA -3'.

Double delta $\mathrm{Ct}$ analysis was used to calculate the mRNA levels. The assay was done in triplicate for each sample.

\section{Teratoma Formation Assay}

For teratoma induction, one million of ESCs were injected intramuscularly into immunodeficient nude mice. For $\mathrm{Mcl}-I^{\text {flox/flox }} \mathrm{Cre}^{\mathrm{ERT} 2} \mathrm{ESCs}$, tamoxifen or vehicle gavage was performed one week after ESCs injection, five days in a row, to delete the endogenous MCL-1. Tumor growth was observed and palpated every day. When any of the tumors reached around twenty percent of surface area of the recipients, all the teratomas were harvested at the same time. Images of teratomas were taken and pieces of teratoma tissues were cut for histology, PCR or immunoblot analysis.

\section{Histology}

Teratoma tissues were fixed in $10 \%$ neutral buffered formalin, embedded in paraffin and cut into 4-um thickness of sections by using HM500M cryostat (Microm). The embedded sections were stained by hematoxylin and eosin (H\&E) stain. Three germ layer tissues of teratoma samples were reviewed by the pathologist Dr. Jerold Rehg at St. Jude Children's Research Hospital.

\section{Cell Death Assay}

F12 ESCs or $M c l-I^{\text {flox/flox }} \mathrm{Cre}{ }^{\text {ERT2 }}$ MEFs stably expressing murine stem cell virus MSCV-puro vector, WT MCL-1, ATG1 or M92 grown in the presence of tamoxifen for 48 hours were treated with staurosporine at increasing doses for 16 hours (Calbiochem). Cell viability was determined by Annexin-V-APC and propidium iodide (PI) (BD Biosciences) staining through flow cytometry FACSCantoII (BD Biosciences).

\section{Subcellular Fractionation and Proteolysis of Heavy Membrane Fraction}

ESCs or MEFs were swollen in ice-cold mitochondria isolation (MIB) buffer (200 $\mathrm{mM}$ mannitol, $68 \mathrm{mM}$ sucrose, $10 \mathrm{mM}$ HEPES-KOH at pH 7.4, $10 \mathrm{mM} \mathrm{KCl}, 1 \mathrm{mM}$ 
EDTA, $1 \mathrm{mM}$ EGTA, 0.1\% BSA) containing protease inhibitor cocktail (PIC) for 30 minutes and then homogenized 30 times with a 30 gauge needle. The non-lysed cells and nuclei were sedimented by centrifugation at $600 \mathrm{~g}$ for 10 minutes and disposed. The collected supernatant was centrifuged at $5,500 \mathrm{~g}$ for 15 minutes and the pellet was the heavy membrane fraction. The supernatant again was centrifuged at 100,000g for 30 minutes and the soluble fraction contained the purified cytosolic proteins.

The isolated heavy membrane (HM) fraction, mainly mitochondria, was measured by Bradford assay for protein concentration. HM (40 ug) were set up with the following treatment: proteinase $\mathrm{K}(0.2 \mathrm{mg} / \mathrm{ml}), 20 \mathrm{mM} \mathrm{KCl}$ (osmotic shock), proteinase $\mathrm{K}(0.2$ $\mathrm{mg} / \mathrm{ml}$ ) plus $20 \mathrm{mM} \mathrm{KCl}$ (osmotic shock), 1\% SDS (detergent for membrane solubilization) for 60 minutes on ice. Proteins were further precipitated by tricholoroacetic acid (TCA) (Sigma) and subjected to immunoblotting analysis.

\section{Zygote Injection}

Freshly fertilized E0.5 post-coital single cell embryos (zygotes) were obtained from freshly weaned ovulated C57BL/6J mice. Recovered zygotes were cultured until both pronuclei were microscopically visible. Approximately 150-200 zygotes were transferred to a microdrop of M2 medium (Sigma-Aldrich) on a microinjection slide overlaid with mineral oil and the slide was placed on an inverted microscopy. Microinjection pipettes were filled with $100 \mathrm{ng} / \mathrm{ul}$ guide RNA with scaffold, $50 \mathrm{ng} / \mathrm{ul}$ Cas 9 mRNA and $200 \mathrm{ng} / \mathrm{ul} \mathrm{ssODN}$. Each embryo was held with light suction by a holding pipette and rotated until one of the two pronuclei was visible. Then, mRNA mixture was injected into the cytoplasm and DNA into one pronucleus. Successful injection of a few picoliters (pl) of solution was observed by the visible swelling of the pronucleus or presence of a jet stream in the cytoplasm. Microinjected zygotes were surgically transferred to the infundibula of 0.5-day-post-coital pseudopregnant foster mothers on the same day, 20 to 25 zygotes per recipient. Full-term pups were obtained by natural labor about 19 days later. All the zygote microinjection procedures were carried out by the transgenic core facility at St. Jude Children's Research Hospital.

\section{DNA Deep Sequencing}

DNA of toes from zygote injection founders was collected. Endogenous Mcl-1 amplicons of exon 1 around start codon were amplified by the same $\mathrm{Mcl}-1$ forward and reverse primers described earlier. Amplicons from different samples were mixed and barcoded by another round of PCR using a Nextera Index Kit and the adaptor primers used were Nextera forward 5'- TCGTCGGCAGCGTCAGATGTGTATAAGAGACAG 3' and reverse 5'- GTCTCGTGGGCTCGGAGATGTGTATAAGAGACAG -3'. The library prepared from the final PCR products of indexed samples was subjected to a 300 bp-paired-end sequencing with Illumina Miseq for the targeted region. After quality control, reads of each sample were aligned to the sequences of targeted region and compared with the same sequencing run generated from control sample with more than 
10,000x depth of coverage. After deep sequencing, an in-house Perl script was used to analyze and summarize the allele frequencies of $M c l-1$ variants.

\section{Microarray Analysis and GSEA}

Affymetrix Mouse Gene 2.0 ST arrays were used to assay gene expression analysis from RNA samples. $\mathrm{Mcl}-\mathrm{I}^{\text {flox/flox }} \mathrm{Cre}^{\text {ERT2 }} \mathrm{ESCs}$ treated with tamoxifen for 48 hours were statistically tested by unequal variance $t$ test to the DMSO control ( 3 replicates). Gene set enrichment analysis (GSEA) (version 2.3.3) was performed by comparing $\mathrm{Mcl}-1^{\text {flox/flox }} \mathrm{Cre}^{\text {ERT2 }}$ ESCs treated with tamoxifen versus DMSO control.

\section{Statistics}

Growth curves significance was calculated by using the two-tailed Student $t$ test and $p$ values are denoted in the figure legends. GSEA comparing $M c l-l^{\text {flox/flox }} \mathrm{Cre}^{\text {ERT2 }}$ ESCs treated with tamoxifen versus DMSO control was statistically calculated by unequal variance $t$ test. 


\section{CHAPTER 3. RESULTS}

\section{CRISPR-Cas9 Targeting of Genomic $\mathrm{Mcl}-1$ to Generate $\mathrm{Mcl}-1$ Mutants}

To target genomic $M c l-1$ by CRISPR-Cas9, we designed 9 individual guide RNAs around the start codon of $\mathrm{Mcl}-1$ and each of the gRNAs was directly upstream of a requisite 5'-NGG. A single-stranded DNA oligonucleotide ( $\mathrm{ssODN)}$ containing both 5' and 3' homologous arms and amino acids 10, 11 mutations was provided as a repair template. Our preliminary data suggested that mutagenesis of amino acids 10, 11 from isoleucine, glycine to aspartic acid abrogates MCL-1's importation to the mitochondrial matrix, only maintaining its anti-apoptotic species. For in vitro targeting, we chose embryonic stem cells (ESCs) because ESCs form self-renewing compact colonies from single cells, which allows us to screen individual mutation, and ESCs are pluripotent cells that can differentiate to three germ cell layers: ectoderm as neurons, mesoderm as cardiomyocytes, and endoderm like gut tissues. More importantly, when injected into blastocysts, those blastocysts can be transferred into foster mothers to make chimeric recipient pups.

We transfected the F12 ESCs with our all-in-one CRISPR-Cas9-GFP plasmid as well as an ssODN containing amino acids 10,11 mutations as the repair template, and sorted GFP positive cells. The CRISPR-Cas9 transfected GFP positive cells were seeded at low density 20,000 cells in $10 \mathrm{~cm}$ dish to grow as single cell clones. The left over targeted pool of cells were first analyzed by Surveyor endonuclease assay T7E1, which is an enzyme mismatch cleavage assay used to detect small mismatches, insertions or deletions. By comparing the amplicons in the presence or absence of T7E1, WT control showed no observable cleaved band (Figure 3-1A). Among the 9 individual crRNAs as well as a paired CRISPR-RNA (crRNAs), four of them, crRNA-F3, crRNA-R1, crRNAR2, crRNA-R3, showed obvious cleavages, indicating the presence of mutant DNAs. The mutation efficiencies were roughly estimated by the densitometry of decreased full-length band in the presence of T7E1 divided by the full-length band in the absence of T7E1 enzyme (Figure 3-1A). Immunoblotting of the targeted pool cells showed reduced expression levels of full-length MCL-1 in crRNA-F3, crRNA-R1, crRNA-R2, and crRNA-R3, which is consistent with T7E1 assay (Figure 3-1B).

After the single cells grew out, we extracted the DNA and performed PCR for the amplicons from ESC clones from the most efficient four gRNAs. All successfully amplified fragments were sent for Sanger sequencing. Total mutation efficiencies ranged from $75 \%$ to $96.2 \%$ among the four gRNAs and bi-allelic mutation efficiencies were from $31.2 \%$ to almost 90\% (Table 3-1). MCL-1 amino acids 10, 11 replacement mutant $\left(\mathrm{CS}^{10,11} \mathrm{MCL}-1\right)$ was not generated during our in vitro screening. We hypothesize is that the bi-allelic mutation efficiency was high and that cells were inclined to repair through the NHEJ pathway. 
A

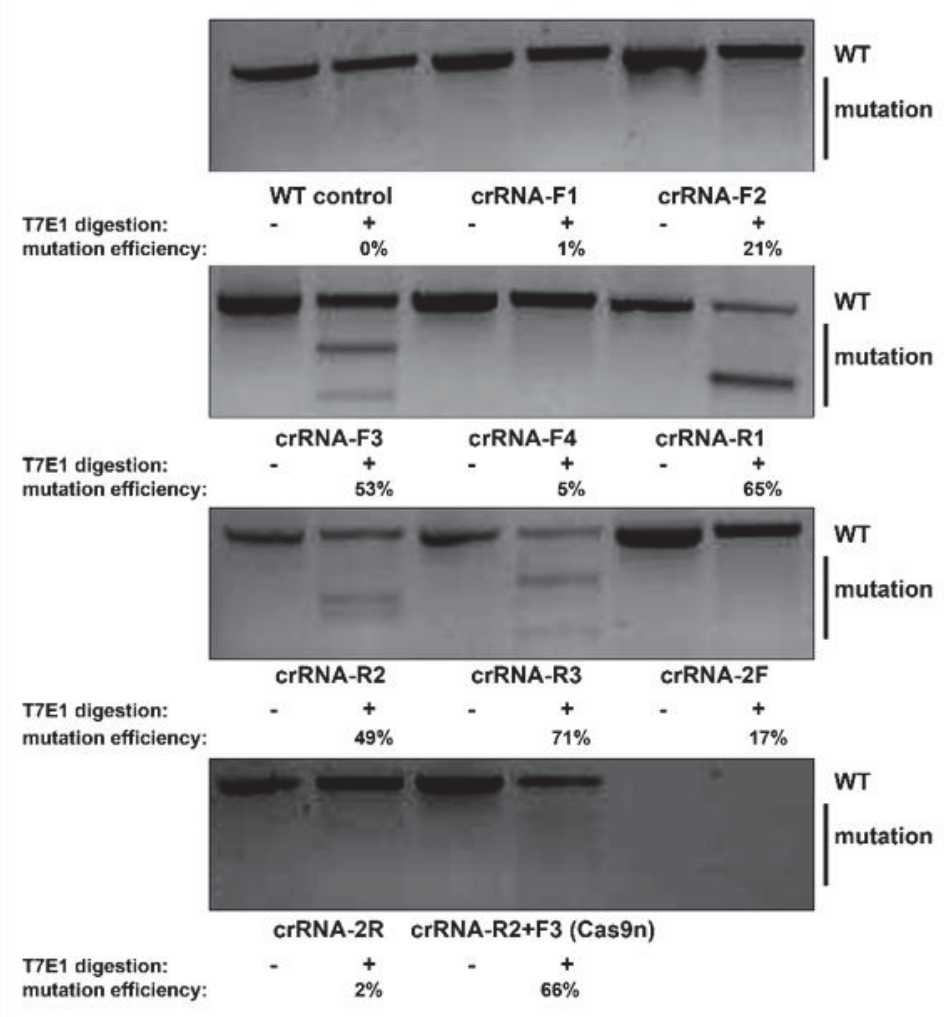

B

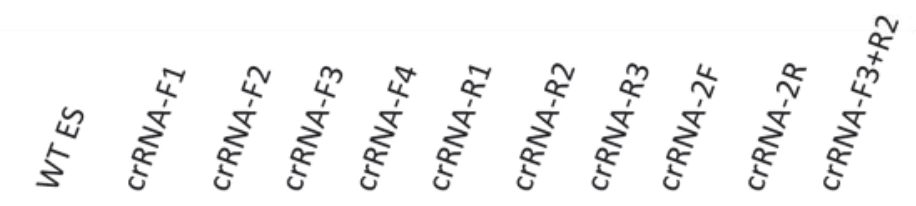

MCL-1

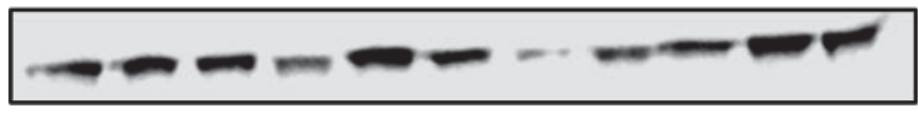

$\alpha$-Actin

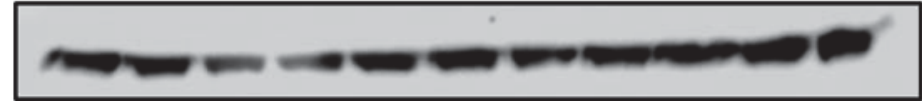

Figure 3-1. Pool validation of CRISPR-Cas9 targeting efficiency

(A) PCR amplified targeted amplicons from each guide RNA pool ESCs were treated in the absence or presence of endonuclease T7E1. The mutation efficiency was calculated by the ratio of decrease full-length band density divided by untreated full-length band density estimated by densitometry. (B) Expression level of full-length MCL-1 in each guide RNA pool ESCs by immunoblotting. $\alpha$-Actin was used as loading control. 
Table 3-1. Summary of F12 ESCs transfected with CRISPR-Cas9 targeting on genomic $\mathrm{Mcl}-1$

\begin{tabular}{cccccc}
\hline $\begin{array}{c}\text { CRISPR } \\
\text { RNA }\end{array}$ & $\begin{array}{c}\text { Clones } \\
\text { Screened }\end{array}$ & $\begin{array}{c}\text { Clones } \\
\text { Amplified }\end{array}$ & $\begin{array}{c}\text { Total } \\
\text { Efficiency }\end{array}$ & $\begin{array}{c}\text { Mono-allelic } \\
\text { Efficiency }\end{array}$ & $\begin{array}{c}\text { Bi-allelic } \\
\text { Efficiency }\end{array}$ \\
\hline crRNA F3 & 48 & 35 & $90 \%$ & $10 \%$ & $80 \%$ \\
crRNA R1 & 48 & 25 & $75 \%$ & $43.8 \%$ & $31.2 \%$ \\
crRNA R2 & 48 & 16 & $75 \%$ & $33.3 \%$ & $41.7 \%$ \\
crRNA R3 & 48 & 27 & $96.2 \%$ & $7.7 \%$ & $88.5 \%$ \\
\hline
\end{tabular}

DNAs extracted from single ESC clones from the four highest mutation efficiency guide RNA groups (F3, R1, R2, and R3), validated from previous T7E1 and pool immunoblotting. DNAs were amplified for the CRISPR-Cas9 targeted amplicons and sent for Sanger sequencing. Homozygous mutations were determined by comparison to mouse $M c l-1$ genomic sequences. Heterozygous mutations were determined by filtering out the known sequence of the wild-type allele. 
At the protein level, large deletion on both alleles disrupting start codon of $\mathrm{Mcl}-1$ all generated a truncated form of MCL-1 rather than a complete knock out. Full-length MCL-1 is around $38 \mathrm{kD}$ and the truncated mutant was around $30 \mathrm{kD}$ (Figure 3-2A). To reveal whether $30 \mathrm{kD}$ starts from the second methionine initiation, we performed mass spectrometry (MS) N-terminal identification. Protein lysates of the enriched heavy membrane from Wild-type (WT) and $30 \mathrm{kD}$ mutant ESCs were loaded on SDS-PAGE gel and the gel bands around $38 \mathrm{kD}$ for WT and $30 \mathrm{kD}$ for mutant ESCs were cut for identification (Figure 3-2B). The proteolytic fragments of the N-terminus were identified according to their mass-to-charge ratio. The data indicate that when disrupting the start codon of $\mathrm{Mcl}-1$ on both alleles, MCL-1 will initiate translation through the second methionine (M92) and produce a $30 \mathrm{kD}$ truncated protein (Figure 3-2C and D).

\section{Mutant ESCs Show a "Sick" Phenotype and Fail to Differentiate to Broad Lineages}

Phenotypically, when seeding equal number of cells during culture, WT ESCs presented as big, healthy clones. As WT control we used the CRISPR-Cas9 targeted GFP negative sorted cells (Figure 3-3). However, $30 \mathrm{kD}$ ESCs presented as much smaller clones with low attachment (Figure 3-3).

To evaluate the pluripotency as well as the differentiation capacity of the mutant ESCs, embryoid body (EB) assay was performed. During the routine culture of ESCs in the presence of leukemia inhibitory factor (LIF) which is used to maintain ESCs' pluripotency, ESCs present as typical compact clones with undifferentiated cells. EBs are generated by separating the undifferentiated ESCs into hanging drops and transferred to ultralow attachment dishes lacking LIF. Cells will aggregate at the bottom of the drop. In the absence of LIF, the well-organized EBs will spontaneously differentiate toward the different germ lineages. They begin with a single extraembryonic endoderm layer which is BMI-1, DAB-2 positive and extraembryonic endoderm surrounds the pluripotent epiblast cells which are OCT-4 positive. Other cells within the inner cell mass are composed of three germ cell lineages, ectoderm, mesoderm, endoderm, as well as the apoptotic cells.

In response to the differentiation signals (absence of LIF), EBs often form cystic cavities, where the interior cells undergo apoptosis while the other cells migrate as well as differentiate to specific lineages. Therefore, cavitation is the relatively early event and the characteristic feature of the EBs undergoing differentiation. On day 4, we started to observe the beginning of a cavity in the WT control EBs, represented by the bright, transparent regions. This was not found among the different EBs with $30 \mathrm{kD}$ MCL-1 protein (Figure 3-4). In order to define whether it is a delay or a completely failed differentiation, EBs were harvested at day 10 and immunofluorescent (IF) staining was performed on the cryosections of EBs to look at the markers for extraembryonic endoderm as well as epiblast cells. The IF data suggested that DAB-2, an extraembryonic endoderm marker, was expressed at the periphery of WT EBs, which surrounded the 
Figure 3-2. Mass spectrometry N-terminal identification of mutant MCL-1

(A) Immunoblotting of the enriched membrane protein fraction from wild-type MCL-1 and mutant $30 \mathrm{kD}$ MCL-1 mouse ESCs was performed to verify MCL-1 protein existence and its localization against the protein ladder (BenchMark, Life Technologies). (B) SDSPAGE of these two samples to excise the gel bands (boxed) for identification by mass spectrometry (MS). (C) The amino acid sequence of mouse MCL-1. The traditional and the hypothetical methionine " $\mathrm{M}$ " at the translation initiation sites were highlighted. The peptide to be identified by MS was underlined. (D) The mass spectrum of the N-terminal peptide of the mutant $30 \mathrm{kD}$ MCL-1. Product ions (fragments from the peptide) were indicated by arrows with measured mass-to-charge ratio $(\mathrm{m} / \mathrm{z})$. All matched product ions were also shown in the peptide. Note: the methionine of this N-terminal peptide was removed, and the next amino acid alanine was acetylated. 

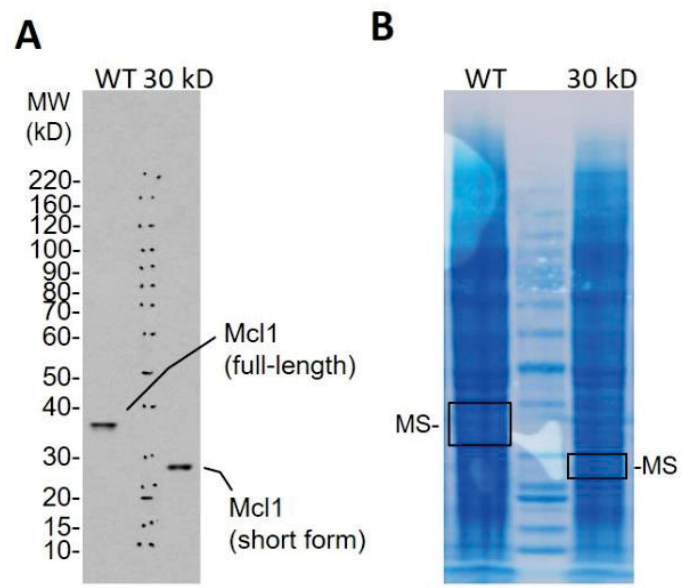

\section{C}

MFGLRRNAVI GLNLYCGGAS LGAGGGSPAG ARLVAEEAKA RREGGGEAAL LPGARVVARP PPVGAEDPDV TASAERRLHK SPGLLAVPPE EMAASAAAAI VSPEEELDGC EPEAIGKRPA

VLPLLERVSE AAKSSGADGS LPSTPPPPEE EEDDLYRQSL EIISRYLREQ ATGSKDSKPL GEAGAAGRRA LETLRRVGDG VQRNHETAFQ GMLRKLDIKN EGDVKSFSRV MVHVFKDGVT NWGRIVTLIS FGAFVAKHLK SVNQESFIEP LAETITDVLV RTKRDWLVKQ RGWDGFVEFF HVQDLEGGIR NVLLAFAGVA GVGAGLAYLI $\mathrm{R}$

D

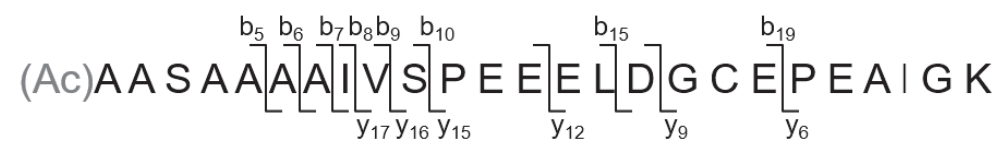

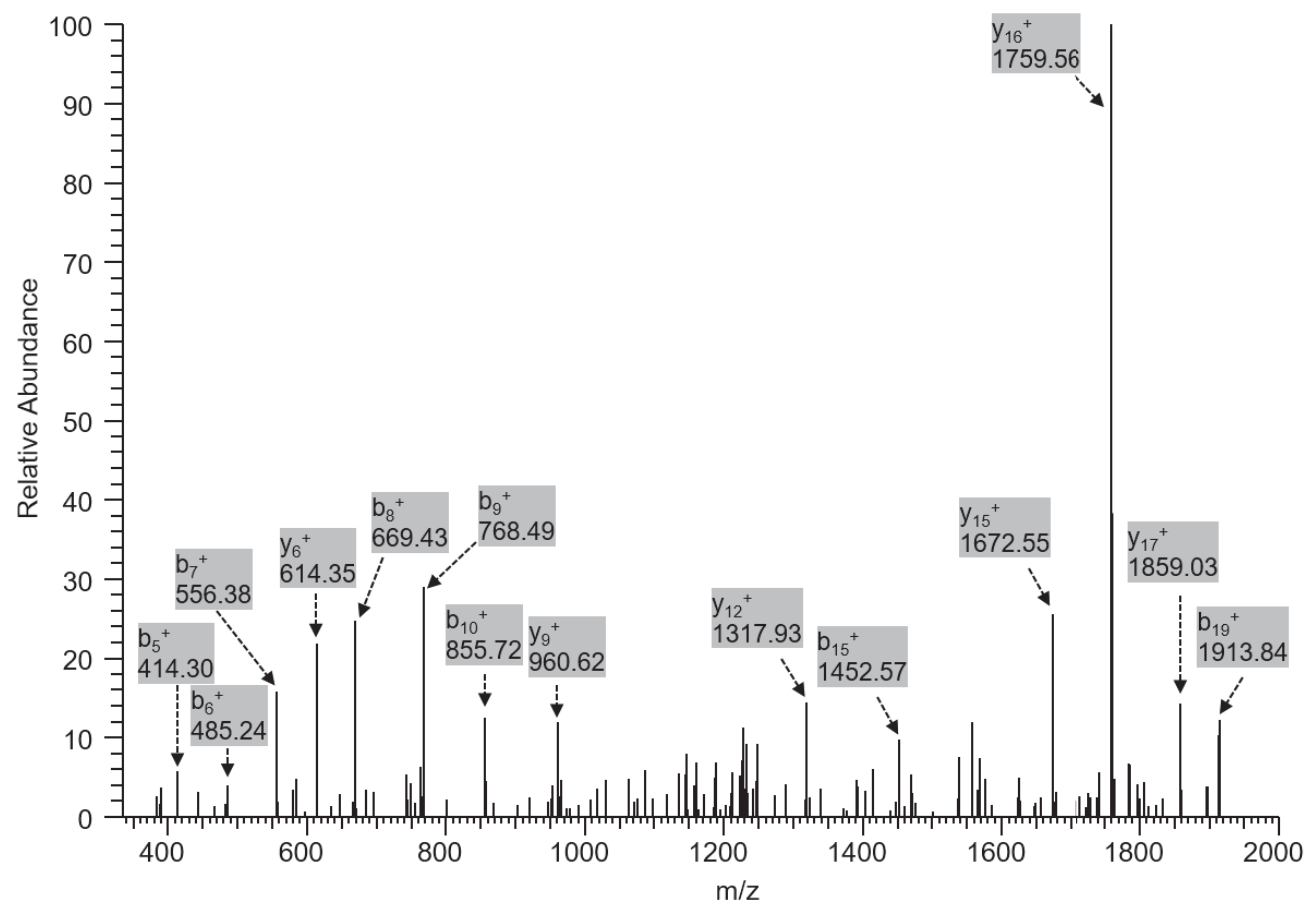


Figure 3-3. Mutant ESCs present a "sick" phenotype in culture

Wild-type ESCs and different lines of $30 \mathrm{kD}$ mutant ESCs $(\mathrm{Cl} 005, \mathrm{Cl} 016, \mathrm{Cl} 061, \mathrm{Cl}$

071, and $\mathrm{Cl} \mathrm{098)} \mathrm{were} \mathrm{seeded} \mathrm{as} \mathrm{equal} \mathrm{number} \mathrm{of} \mathrm{cells} \mathrm{in} \mathrm{culture.} \mathrm{The} \mathrm{phenotype} \mathrm{of} \mathrm{cells}$ were observed and captured by inverted microscopy (Nikon Eclipse TS100). 


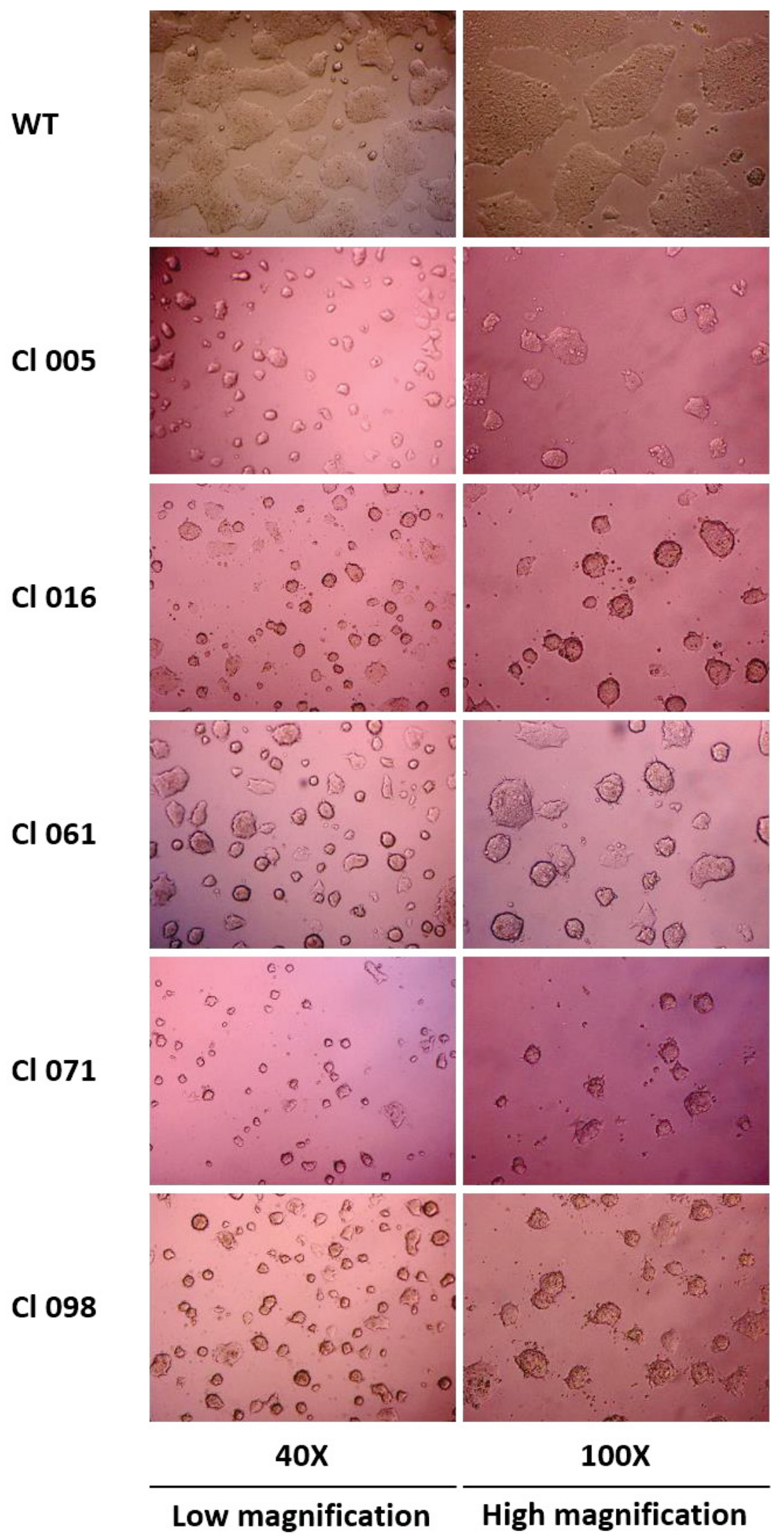




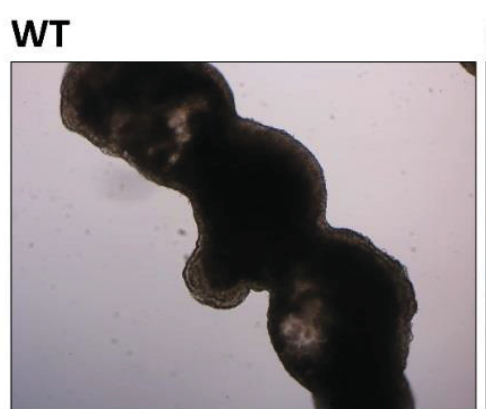

$\mathrm{Cl} 005$
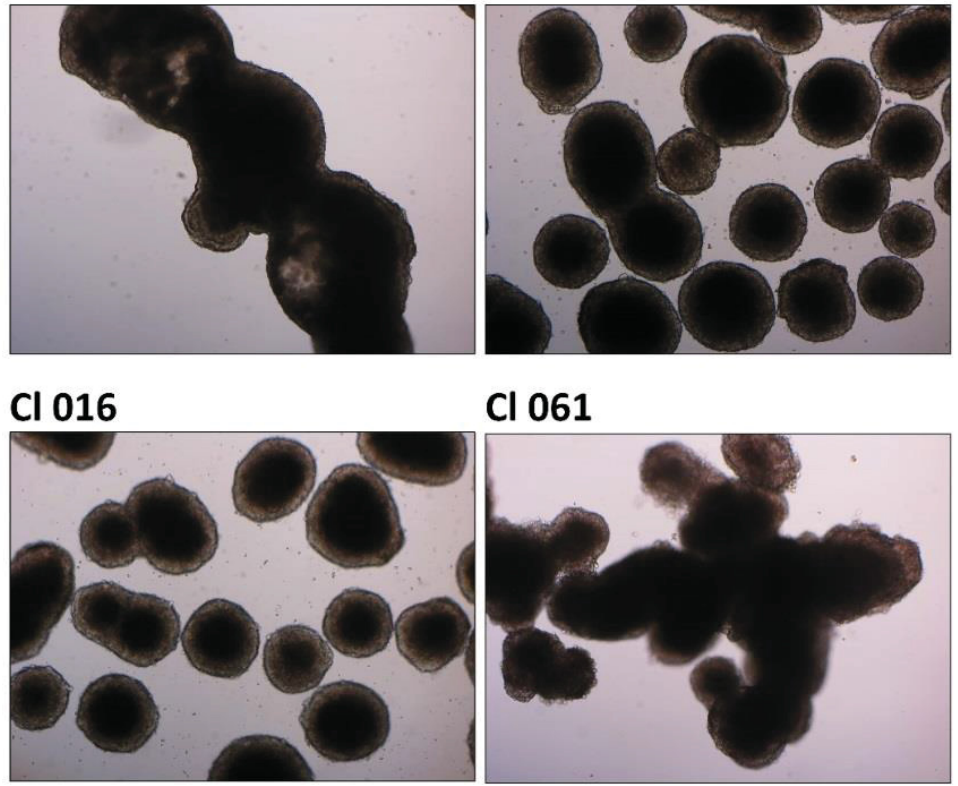

\section{$\mathrm{Cl} 016$}

\section{$\mathrm{Cl} 061$}

Cl 071
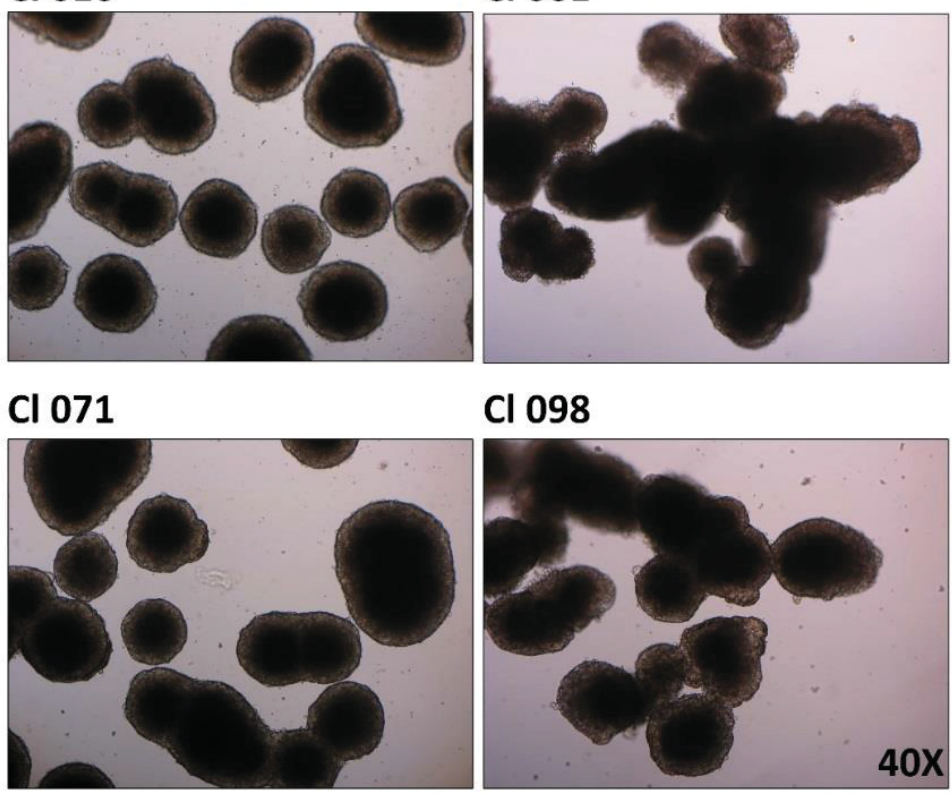

$\mathrm{Cl} 098$

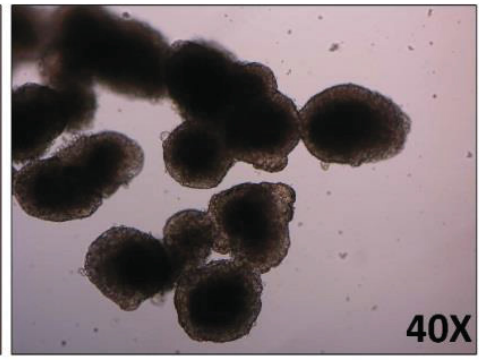

Figure 3-4. Embryoid body spontaneous differentiation of ESCs

WT or different lines of mutant $30 \mathrm{kD}$ ESCs $(\mathrm{Cl} 005, \mathrm{Cl} 016, \mathrm{Cl} 061, \mathrm{Cl} 071$, and $\mathrm{Cl}$ 098) were dissociated into hanging drops and transferred to ultralow attachment dishes in the absence of leukemia inhibitory factor (LIF). At day 4, embryoid body (EB) formation was observed and captured by inverted microscopy (Nikon Eclipse TS100). 
OCT-4 (a pluripotent marker) positive epiblast cells. Overlay showed a nice ring, halolike structure. However, for the $30 \mathrm{kD}$ mutant EBs, the OCT-4 positive cells were observed, but few DAB-2 positive cells located at the periphery, indicating a defect of 30 $\mathrm{kD}$ ESCs to differentiate to extraembryonic endoderm, which is consistent with the trophectoderm defect during peri-implantation stage of $M c l-1$-null embryos

(Figure 3-5A). RNAs from WT and $30 \mathrm{kD}$ mutant EBs were extracted at day 4 and quantitative real-time PCR (qPCR) was performed to assess the markers from each lineage. qPCR suggested a broader differentiation defects in $30 \mathrm{kD}$ mutant EBs compared with WT, showing dramatic decreased levels of Sry-related high-mobility group box 7 (SOX7), another extraembryonic endoderm marker, fibroblast growth factor 5 (FGF5), a ectoderm marker, paired related homeobox 1 ( $\mathrm{mHOX}$ ), a mesoderm marker, and Indian hedgehog (IHH), an endoderm marker (Figure 3-5B). These data, taken together, indicate that N-terminal truncated MCL-1 ESCs present as a sick phenotype and fail to differentiate to broad lineages, including extraembryonic endoderm, ectoderm, mesoderm, and endoderm.

Teratoma formation assay is an in vivo assay to test the pluripotency of ESCs and their capability of differentiation. One million WT control ESCs or $30 \mathrm{kD}$ mutant ESCs were injected into the flank of nude mice: two out of three WT ESCs gave rise to big teratomas 25 days post-implantation (Figure 3-6A). However, $30 \mathrm{kD}$ ESCs failed to give rise to any size tumors at the same given time (Figure 3-6A). In order to test whether the effect is MCL-1 specific and not random off-target effect in one special ES clone, one million of the targeted pool ESCs were injected into the flank of nude mice. Three out of four low efficient CRISPR-targeted pool ESCs gave rise to teratomas 25 days post implantation, while only one out of four high efficient pool cells produced a tiny tumor when harvested at the same time (Figure 3-6A). Hematoxylin and eosin (H\&E) stain of WT control teratoma showed tissues from all three germ lineages, surface ectoderm as the keratinized epithelial, neural ectoderm as neuronal rosettes, mesoderm such as cartilages as well as the striated muscles, and endoderm showing as the respiratory epithelial with mucus cells and cilia (Figure 3-6B). These data, consistent with the in vitro differentiation assay, suggested that $\mathrm{N}$-terminal truncated mutants ESCs fail to differentiate to broad germ lineages in vivo.

\section{Mutant MCL-1 Is Capable of Antagonizing Cell Death and Localizes to the Outer Mitochondrial Membrane}

MCL-1 is well-known for its anti-apoptotic function, therefore it is important to test whether $30 \mathrm{kD}$ mutant ESCs can antagonize cell death. Staurosporine, a broad kinase inhibitor capable of inducing apoptosis, was added at different doses on WT ESCs and different lines of $30 \mathrm{kD}$ mutants. The curves representing the percentage of annexin positive cells at different doses of staurosporine didn't show obvious differences among WT ESCs and several $30 \mathrm{kD}$ ESC lines (Figure 3-7A). The subcellular localization data showed that WT MCL-1 and $30 \mathrm{kD}$ mutant MCL-1 were enriched at the heavy membrane (HM), indicating their mitochondrial localization (Figure 3-7B). Further, proteinase K 
A

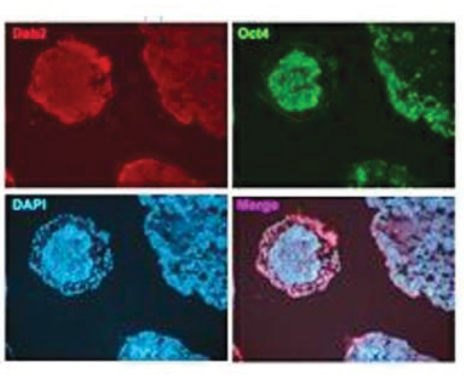

WT EB day 10

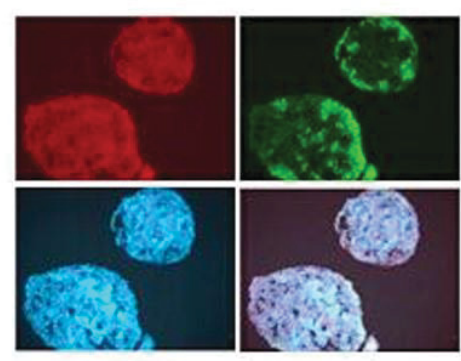

$\mathrm{Cl} 005$ EB day 10

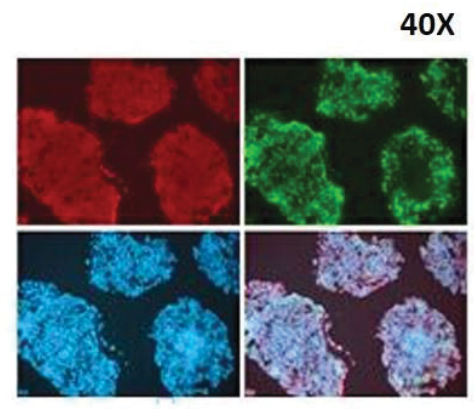

Cl 061 EB day 10

B
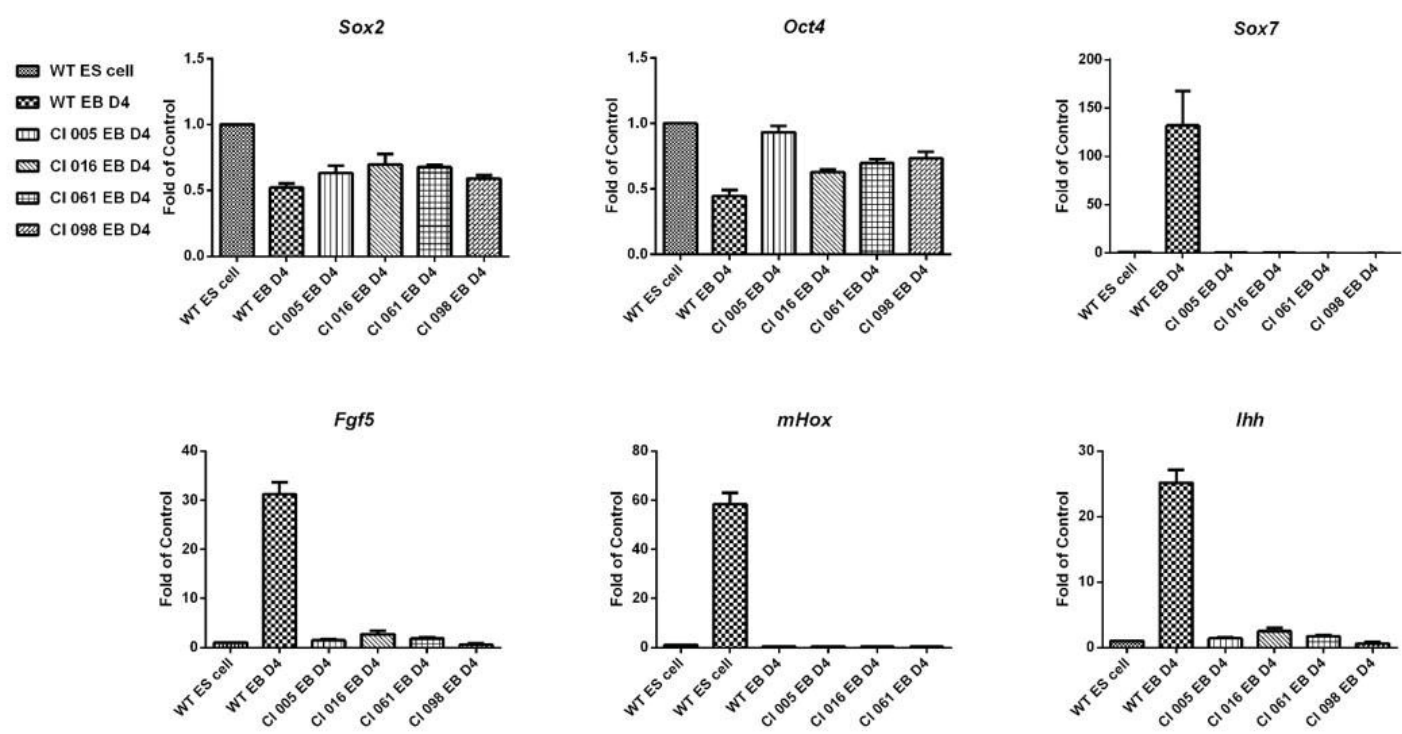

Figure 3-5. $30 \mathrm{kD}$ mutant ESCs show broad differentiation defects

(A) Expression of DAB-2 and OCT-4 in the embryoid body day 10. Cryosections of spontaneous differentiation embryoid bodies (WT versus two lines of $30 \mathrm{kD}$ ) at day 10 were immunostained with anti-DAB-2 and anti-OCT-4 antibodies. Sections were counterstained with the DNA dye DAPI. All images were captured by using a microscopy (Zeiss Axioskop II Plus). (B) Real-time PCR with fast SYBR green (Bio$\mathrm{Rad}$ ) detection was performed by using QuantStudio 7 Flex (Life technologies). Primers used were Sox2 (pluripotency marker), Oct4 (pluripotency marker), Sox7 (extraembryonic endoderm), Fgf5 (ectoderm), mHox (mesoderm), Ihh (endoderm). Double delta $\mathrm{Ct}$ analysis was used to calculate the mRNA levels. The assay was done in triplicate for each sample. Error bars represent the standard error of the mean. 


\section{A}
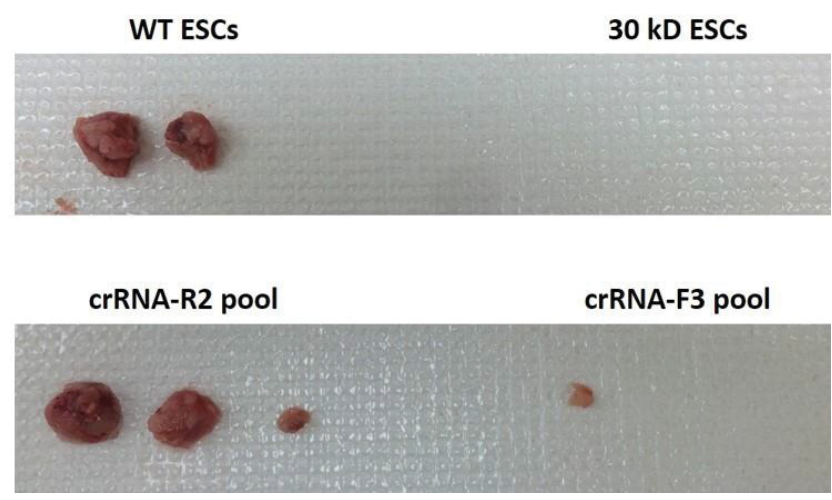

B
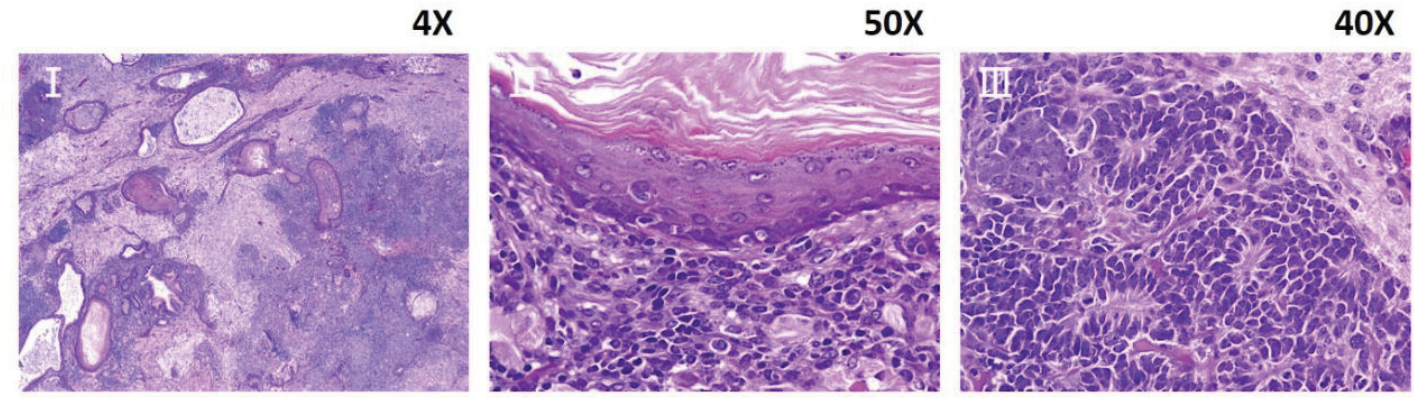

$50 X$
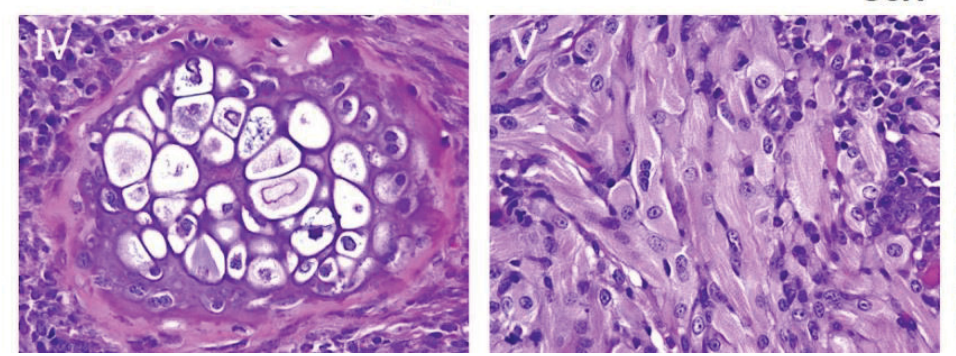

$50 \mathrm{X}$

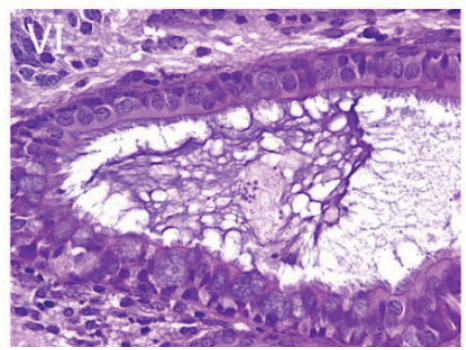

Figure 3-6. $\quad 30 \mathrm{kD}$ mutant ESCs fail to give rise to teratoma

(A) Teratoma formation of WT, $30 \mathrm{kD}$ ESCs and low versus high efficient CRISPR-Cas9 targeted pool ESCs. One million ESCs were injected into the flank of each immunodeficient recipient and tumors were harvested 25 days post implantation. (B) H\&E stain of WT teratomas showed tissues from all three germ layers, I (low magnification), II-VI (high magnification), II (surface ectoderm as the keratinized epithelial), III (neural ectoderm as neuronal rosettes), IV (mesoderm as cartilages), V (mesoderm as striated muscles), and VI (endoderm showing as the respiratory epithelial with mucus cells and cilia). 
A
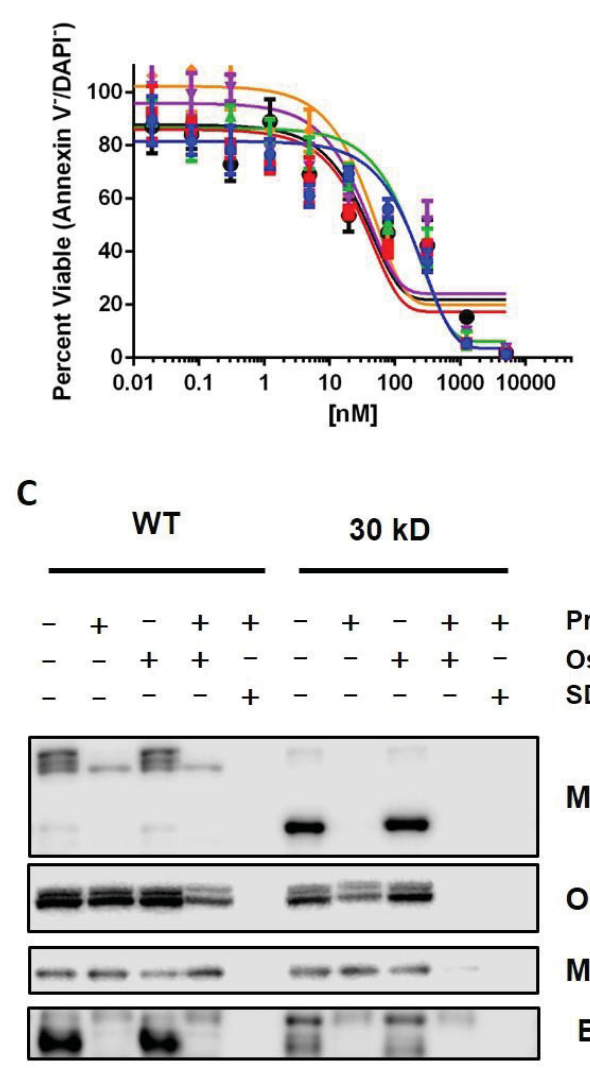

B

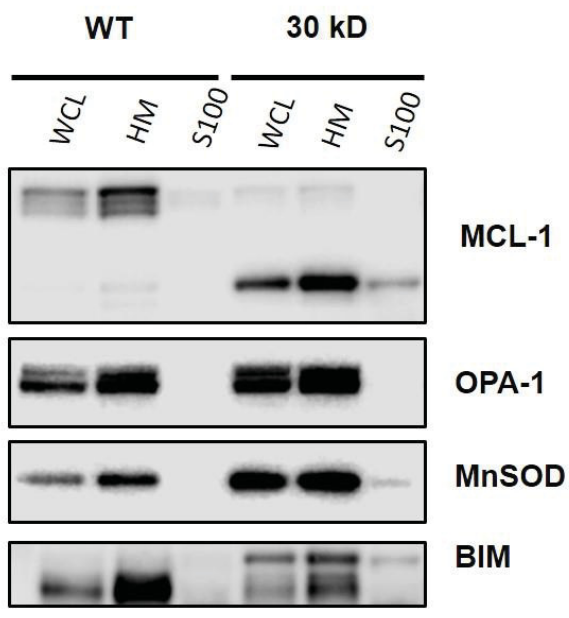

Figure 3-7. $30 \mathrm{kD}$ mutant ESCs retain anti-apoptotic function and reside on the outer mitochondrial membrane

(A) WT ESCs and different lines of $30 \mathrm{kD}$ ESCs were administered indicated doses of staurosporine for 16 hours after which cell death assay by flow cytometry was determined. Bars represent the average of 3 independent experiments and the error bars denote the SEM. (B) Mitochondria enriched, heavy membrane (HM) or cytosol (S100) fractions were isolated from WT and $30 \mathrm{kD}$ ESCs. Whole cell lysates (WCL) acted as a control. Fractions were resolved and immunoblotted for MCL-1. OPA-1, MnSOD, or BIM worked as intermitochondrial membrane, inner matrix or cytosolic marker, respectively. (C) Mitochondria isolated from WT or $30 \mathrm{kD}$ ESCs were subjected to proteinase $\mathrm{K}(\mathrm{PK})$ proteolysis. Osmotic shock was used to disrupt outer mitochondrial membrane (OMM), and detergent SDS was used to disrupt both inner and outer mitochondrial membranes. Lysates were collected and subjected to immunoblot analyses. 
assay was carried out on the isolated mitochondria. Upper and middle bands of MCL-1 were both sensitive to proteinase $\mathrm{K}$ digestion, but the lower band was resistant even under the osmotic shock. This is consistent with our previous finding suggesting that WT MCL-1 retains outer mitochondrial membrane and matrix dual localizations

(Figure 3-7C). However, $30 \mathrm{kD}$ mutant MCL-1 only has one band and was sensitive to proteinase $\mathrm{K}$ cleavage, indicating that it only resides on the outer mitochondrial membrane (Figure 3-7C).

To further evaluate the anti-apoptotic function and the subcellular localization of $30 \mathrm{kD}$ mutant MCL-1, our inducible mouse embryonic fibroblasts (MEFs) were utilized. The second methionine $30 \mathrm{kD}$ mutant MCL-1 was made by either mutating the first methionine to TGA stop code called ATG1, or by truncation of the N-terminal 91 amino acids named M92. MSCV-puro vector, WT MCL-1, ATG1 and M92 were overexpressed to $\mathrm{Mcl}-1^{\text {flox/flox }} \mathrm{CRE}^{\mathrm{ERT} 2} \mathrm{SV} 40$ transformed MEFs and then selected by puromycin. After the stable cell lines were established, they were treated with tamoxifen for 48 hours. Immunoblotting showed the endogenous MCL-1 was completely deleted and WT MCL-1 as well as ATG1, M92 mutant MCL-1 were well expressed (Figure 3-8A). Cell death assay by staurosporine at different doses was repeated. MCL-1-deleted MEFs overexpressing the empty vector were sensitive to death stimuli and dramatic cell death was achieved at relative lower staurosporine doses (Figure 3-8B). MCL-1 deleted MEFs overexpressing either WT MCL-1, ATG1 or M92 exhibited resistance to cell death stimuli (Figure 3-8B). One mechanism of MCL-1's anti-apoptotic role is to bind with the pro-apoptotic death sensor BIM by using its hydrophobic groove to block BAX/BAK activation. Further, BIM immunoprecipitation (IP) experiment revealed that ATG1 and M92 were capable of binding to BIM like the upper anti-apoptotic MCL-1 species (Figure 3-8C). Consistent with the proteinase K assay in ESCs, in the inducible MEFs system WT MCL-1 retained its dual subcellular localization by presenting with both sensitive upper band and resistant lower band to proteinase K. In contrast, both ATG1 and M92 exhibited one band which was sensitive to enzyme digestion, further suggesting its only outer mitochondrial membrane localization (Figure 3-8D).

\section{Establish $M c l-1^{\text {flox/flox }}$ CRE $^{\text {ERT2 }}$ ESCs for Rescue Experiment}

To rescue the differentiation defect from mutant MCL-1, $M c l-1^{\text {flox/flox }} \mathrm{CRE}^{\mathrm{ERT} 2}$ ESCs were derived as well as $M c l-1^{\text {flox/WT }} \mathrm{CRE}^{\mathrm{ERT} 2}$ and $\mathrm{Mcl}-1^{\text {flox/flox }}$ controls. Immunoblotting data suggested endogenous MCL-1 was well deleted 48 hours after tamoxifen treatment on both $\mathrm{Mcl}-1^{\text {flox/flox }} \mathrm{CRE}^{\text {ERT2 }} \mathrm{ESCs}$ and embryoid bodies (EBs) day 4 (Figure 3-9A). $M c l-1^{\text {flox/WT }} \mathrm{CRE}^{\mathrm{ERT} 2}$ treated with tamoxifen showed decreased level of MCL-1 compared with $M c l-1^{\text {flox/WT }} \mathrm{CRE}^{\mathrm{ERT} 2}$ vehicle treated (Figure 3-9A). When seeded equal number of cells in culture in the presence or absence of tamoxifen and followed for growth, tamoxifen treated $\mathrm{Mcl}-\mathrm{I}^{\text {flox/flox }} \mathrm{CRE}^{\mathrm{ERT} 2} \mathrm{ESC}$ stopped expanding in culture while Mcl- $1^{\text {flox/flox }} \mathrm{CRE}^{\text {ERT2 }}$ ESCs untreated as well as $\mathrm{Mcl}-1^{\text {flox/flox }}$ regardless of the presence of tamoxifen showed exponential growth curve (Figure 3-9B). $M c l-1^{\text {flox/WT }} \mathrm{CRE}^{\mathrm{ERT} 2}$ treated with tamoxifen exhibited slower growth rate compared with $\mathrm{Mcl}-1^{\text {flox/WT }} \mathrm{CRE}^{\mathrm{ERT} 2}$ in the 
A

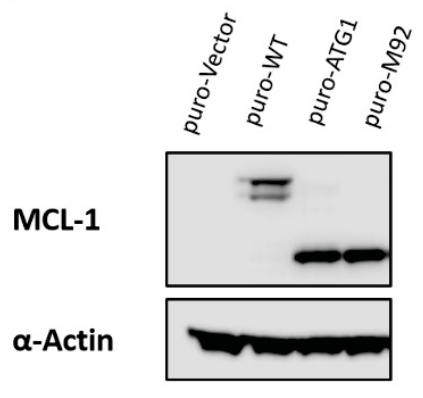

c

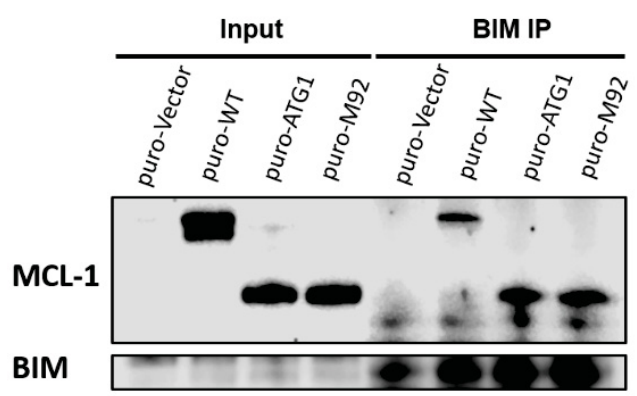

B

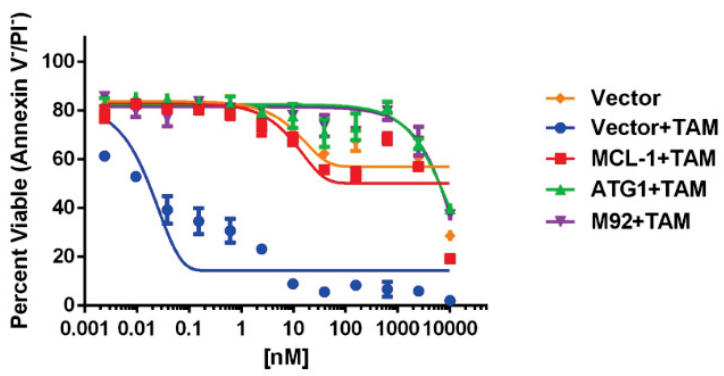

D

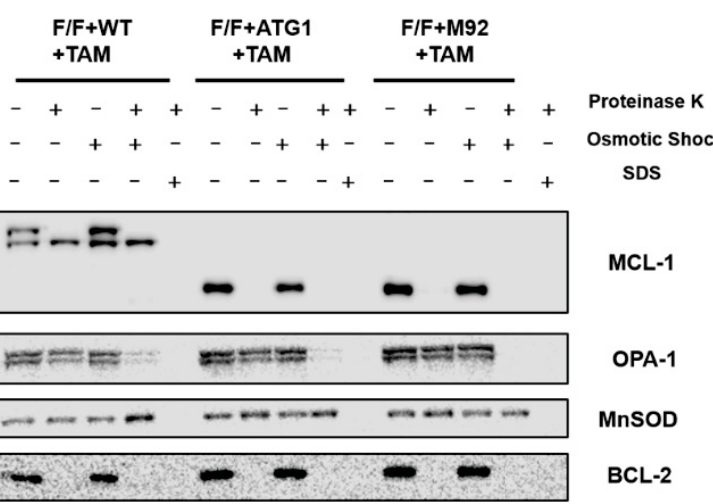

Figure 3-8. Generation of inducible MEFs overexpressing $30 \mathrm{kD}$ mutant for cell death and subcellular localization analyses

(A) Western blot of the $M c l-1^{\text {flox/flox }} \mathrm{CRE}^{\mathrm{ERT} 2} \mathrm{SV} 40$ transformed MEFs stably overexpressing MSCV-puro vector, WT MCL-1, ATG1, and M92 treated with tamoxifen for 48 hours for anti-MCL-1. Anti- $\alpha$-actin acted as a loading control. (B) $M c l-1^{\text {flox/flox }}$ CRE $^{\text {ERT2 }}$ SV40 transformed MEFs stably overexpressing MSCV-puro vector, WT MCL1, ATG1, and M92 treated with tamoxifen for 48 hours were administered indicated doses of staurosporine for 16 hours after which cell death assay by flow cytometry was determined. Bars represent the average of 3 independent experiments and the error bars denote the SEM. (C) Whole cell lysates (WCLs) of $M c l-1^{\text {flox/flox }} \mathrm{CRE}^{\mathrm{ERT} 2} \mathrm{SV} 40$ transformed MEFs stably overexpressing MSCV-puro vector, WT MCL-1, ATG1, and M92 treated with tamoxifen for 48 hours were collected and coupled with anti-BIM rat monoclonal antibody, and then precipitated with protein $\mathrm{A} / \mathrm{G}$-plus agarose.

Immunocomplexes and post-immnoprecipitated supernatants were subjected to immunoblotting for anti-MCL-1 and anti-BIM. (D) Mitochondria isolated from $\mathrm{Mcl}$ $I^{\text {flox/flox }}$ CRE $^{\text {ERT2 }}$ SV40 transformed MEFs stably overexpressing MSCV-puro vector, WT MCL-1, ATG1, and M92 treated with tamoxifen for 48 hours were subjected to proteinase $\mathrm{K}(\mathrm{PK})$ proteolysis. Osmotic shock was used to disrupt outer mitochondrial membrane (OMM), and detergent SDS was used to disrupt both inner and outer mitochondrial membranes. Lysates were collected and subjected to immunoblot analyses. OPA-1, MnSOD, or mouse BCL-2 worked as intermitochondrial membrane, inner matrix or cytosolic marker, respectively. 
A

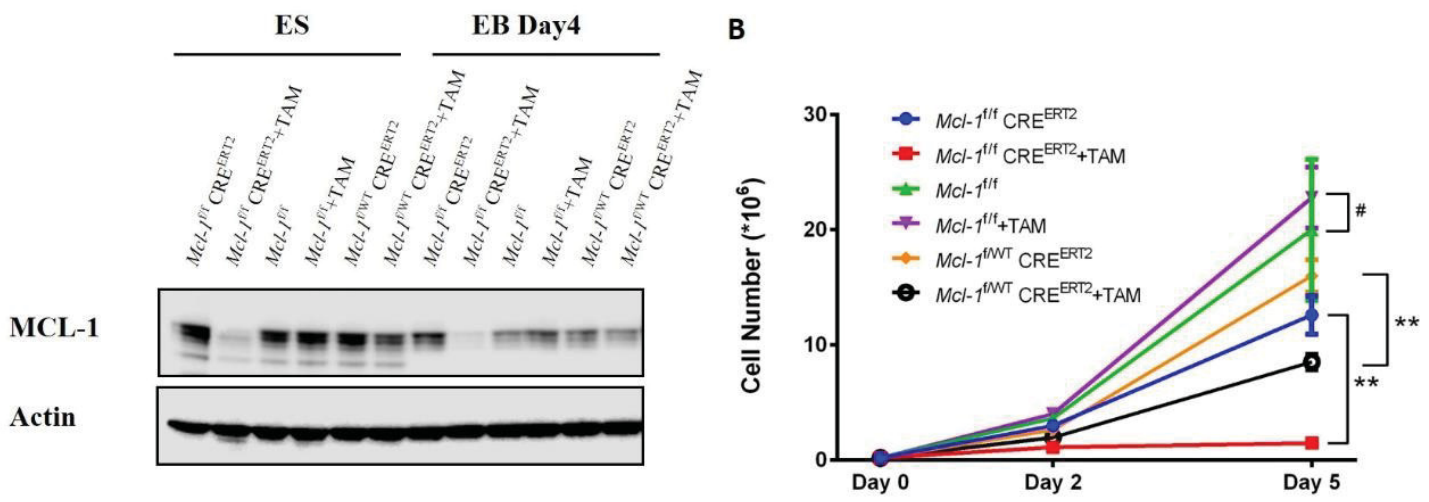

C

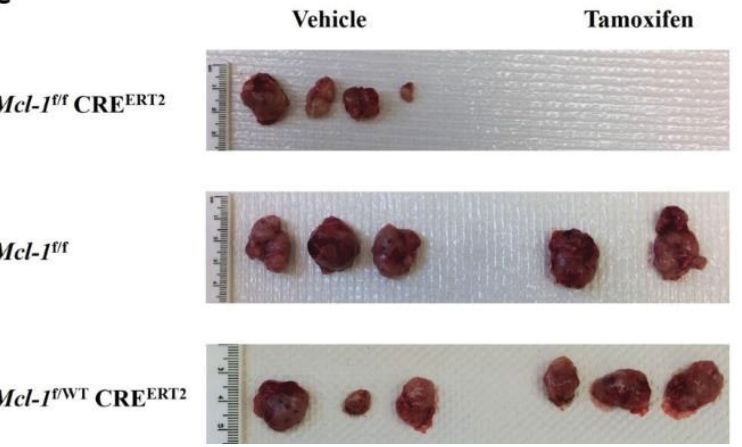

Figure 3-9. Mcl-1-deleted $M c l-1^{\text {flox/flox }} \mathrm{CRE}^{\mathrm{ERT} 2} \mathrm{ESCs}$ fail to proliferate in vitro and give rise to teratoma in vivo

(A) Western blot of the $M c l-1^{\text {flox/flox }} \mathrm{CRE}^{\text {ERT2 }}, M c l-1^{\text {flox/flox }}$, and $M c l-1^{\text {flox/WT }} \mathrm{CRE}^{\mathrm{ERT} 2}$ ESCs in the absence and presence of tamoxifen for 48 hours. Anti- $\alpha$-actin acted as a loading control. (B) $M c l-1^{\text {flox/flox }} \mathrm{CRE}^{\mathrm{ERT} 2}, M c l-1^{\text {flox/flox }}$, and $M c l-1^{\text {flox/WT }} \mathrm{CRE}^{\text {ERT2 }} \mathrm{ESCs}$ in the absence and presence of tamoxifen were seeded equal number of cells in culture and followed and plotted for growth curves. Bars represent the average of 3 independent experiments and the error bars denote the SEM. $\# p>0.05$ and $* * p<0.01$ as determined by two-tailed Student $t$ test. (C) Teratoma formation of $M c l-1^{\text {flox/flox }} \mathrm{CRE}^{\text {ERT2 }}, M c l-1^{\text {flox/flox }}$, and $\mathrm{Mcl}-\mathrm{I}^{\text {flox/WT }} \mathrm{CRE}^{\mathrm{ERT} 2} \mathrm{ESCs}$ in the absence and presence of tamoxifen. One million of each ESCs were injected into the flank of immunodeficient recipient and tumors were palpated every the other day. $\mathrm{Mcl}-\mathrm{I}^{\text {flox/flox }}$ and $\mathrm{Mcl}-\mathrm{I}^{\text {flox/WT }} \mathrm{CRE}{ }^{\mathrm{ERT} 2}$ teratomas regardless of tamoxifen treatment were harvested 25 days post implantation, while $\mathrm{Mcl}-\mathrm{I}^{\text {flox/flox }}$ $\mathrm{CRE}^{\mathrm{ERT} 2}$ teratomas were harvested 39 days post implantation. 
absence of tamoxifen (Figure 3-9B). To test whether the $M c l-1^{\text {flox/flox }} \mathrm{CRE}^{\mathrm{ERT} 2} \mathrm{ESCs}$ we generated retained pluripotency and response to tamoxifen in vivo, one million of ESCs were injected into the flank of nude mice and vehicle or tamoxifen gavage was performed one week post the implantation, five days in a row. All four immunodeficient recipients receiving $\mathrm{Mcl}-\mathrm{I}^{\text {flox/flox }} \mathrm{CRE}^{\text {ERT2 }}$ ESCs treated with vehicle had teratomas at 39 days after implantation. However, none of the recipients transplanted with $\mathrm{Mcl}-1^{\text {flox/flox }} \mathrm{CRE}^{\mathrm{ERT} 2}$ and treated with tamoxifen had observable tumors at the same time (Figure 3-9C). Mcl$1^{\text {flox/WT }} \mathrm{CRE}^{\mathrm{ERT} 2}$ and $\mathrm{Mcl}-1^{\text {flox/flox }}$ ESCs treated either with vehicle or tamoxifen gave rise to big size teratomas at 25 days after injection (Figure 3-9C). These data taken together suggest that MCL-1 is required for ESCs proliferation and differentiation to multiple germ lineages. ESCs with one deleted allele of MCL-1 showed attenuated growth rate in culture.

We further made mutants to dissect MCL-1's two isoforms for ESCs rescue purpose. $\mathrm{CS}^{10,11}$ and $\mathrm{CS}^{33,34} \mathrm{MCL}-1$ were generated by either mutagenesis of amino acids at position 10, 11 from isoleucine, glycine to aspartic acid, aspartic acid or amino acids at position 33, 34 from leucine, valine to aspartic acid, aspartic acid (Figure 3-10A). To generate a MCL-1 mutant that only restricts within matrix, we fused the mitochondrial targeting sequence from ATP synthase to N-terminal 34 amino acids truncated MCL-1, call Matrix MCL-1 (Figure 3-10A). Consistent with our hypothesis, compared with WT MCL-1's dual localization, Matrix MCL-1 only formed one band which was completely resistant to enzyme digestion even under the osmotic shock, suggesting its mitochondrial matrix only localization (Figure 3-10B). Both $\mathrm{CS}^{10,11}$ and $\mathrm{CS}^{33,34} \mathrm{MCL}-1$ were sensitive to proteinase $\mathrm{K}$, therefore they only reside on the outer mitochondrial membrane

(Figure 3-10B). And our previous functional studies indicated that two species of MCL-1 retain different functions due to their distinct localization. Outer mitochondrial membrane localized MCL-1 performs the classical anti-apoptotic function by antagonizing BAX and BAK, while matrix localized MCL-1 does not possess anti-apoptotic activity, but plays a role in maintaining mitochondrial ultrastructure, promoting mitochondrial fusion and fission, supporting the assembly of ATP synthase oligomers and oxidative phosphorylation [38].

To evaluate which MCL-1 function would rescue the defects of ESCs, MSCV-IGFP (MIG) Vector, WT MCL-1, CS ${ }^{10,11}$, Matrix, 30 kD MCL-1 or hBCL-2 was overexpressed onto $\mathrm{Mcl}-1^{\text {flox/flox }} \mathrm{CRE}^{\text {ERT2 }} \mathrm{ESCs}$ by viral transduction. Tamoxifen or vehicle was performed on each of them and cell lysates were harvested at either day 3 and 6 to check the endogenous MCL-1 deletion efficiency as well as the exogenous overexpression level. The $\mathrm{Mcl}-\mathrm{I}^{\text {flox/flox }} \mathrm{CRE}^{\text {ERT2 }} \mathrm{ESCs}$ overexpressing MIG vector showed good deletion efficiency on day 3 after tamoxifen treatment, however at day 6 , the endogenous MCL-1 could not be deleted in the presence of tamoxifen, indicating a long-term selection pressure against MCL-1 loss (Figure 3-11A). This finding is consistent with our previous publication that there was strong selection against MCL-1 loss during BCL-ABL pre-B leukemia transformation in vitro and in vivo [110]. WT, $\mathrm{CS}^{10,11}$, Matrix, $30 \mathrm{kD}$ MCL-1 and hBCL-2 were well-expressed and the endogenous MCL-1 in these groups were well-deleted (Figure 3-11A). When seeded equal number of cells in the presence or absence of tamoxifen in culture, $M c l-1^{\text {flox/flox }} \mathrm{CRE}^{\mathrm{ERT} 2} \mathrm{ESCs}$ 
A

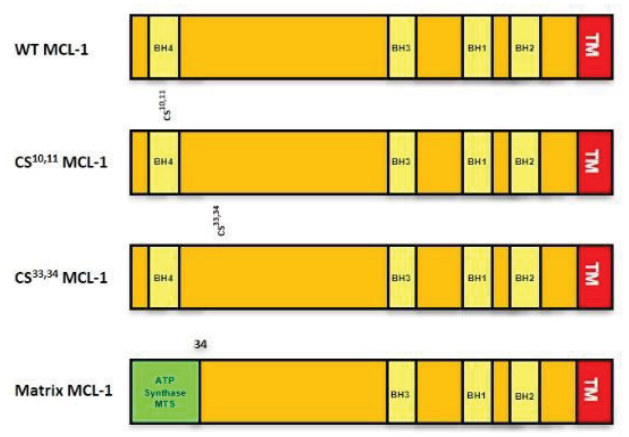

B
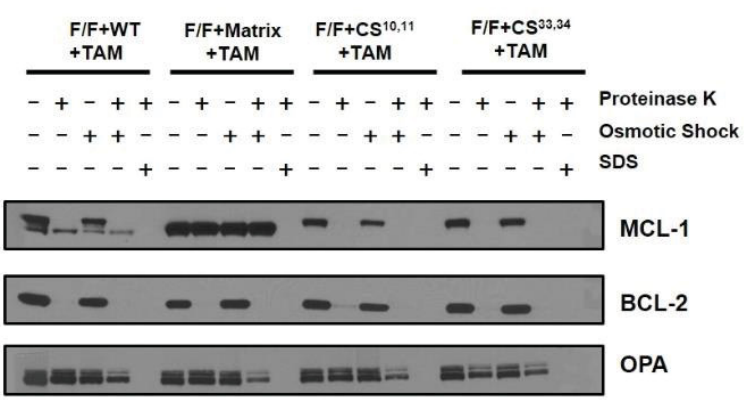

$\because-\infty-\infty-\infty-\infty-\infty$ MnSOD

Figure 3-10. Dissect MCL-1's two isoforms for rescue experiments

(A) Schematic illustration of cleavage sites mutants and Matrix mutant of MCL-1. CS ${ }^{10}$, ${ }^{11}$ and $\mathrm{CS}^{33,34} \mathrm{MCL}-1$ were generated by either mutagenesis of amino acids at position 10 , 11 from isoleucine, glycine to aspartic acid, aspartic acid or amino acids at position 33, 34 from leucine, valine to aspartic acid, aspartic acid. Matrix MCL-1 was generated by fusing the mitochondrial targeting sequence from ATP synthase to N-terminal 34 amino acids truncated MCL-1. (B) Mitochondria isolated from $\mathrm{Mcl}-1$-deleted $\mathrm{Mcl}-\mathrm{I}^{\text {flox/flox }}$ $\mathrm{CRE}^{\text {ERT2 }} \mathrm{SV} 40$ transformed MEFs stably overexpressing MSCV-puro WT MCL-1, CS ${ }^{10}$, ${ }^{11}, \mathrm{CS}^{33,34}$, Matrix MCL-1 treated with tamoxifen for 48 hours were subjected to proteinase $\mathrm{K}(\mathrm{PK})$ proteolysis. Osmotic shock was used to disrupt outer mitochondrial membrane (OMM), and detergent SDS was used to disrupt both inner and outer mitochondrial membranes. Lysates were collected and subjected to immunoblot analyses. OPA-1, MnSOD, or mouse BCL-2 worked as intermitochondrial membrane, inner matrix or cytosolic marker, respectively. 
A

$M c l-1^{\text {ff }} \mathrm{CRE}^{\mathrm{ERT} 2} \mathrm{OE}$

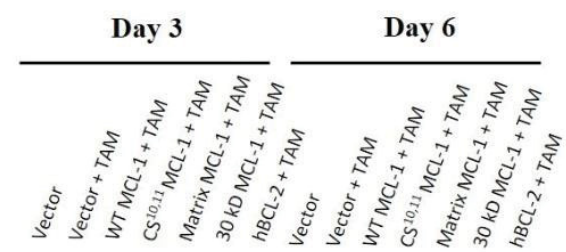

MCL-1

hBCL-2
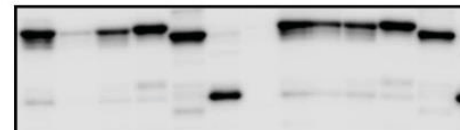

Actin

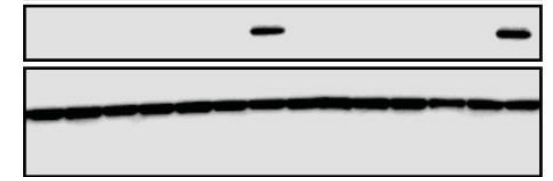

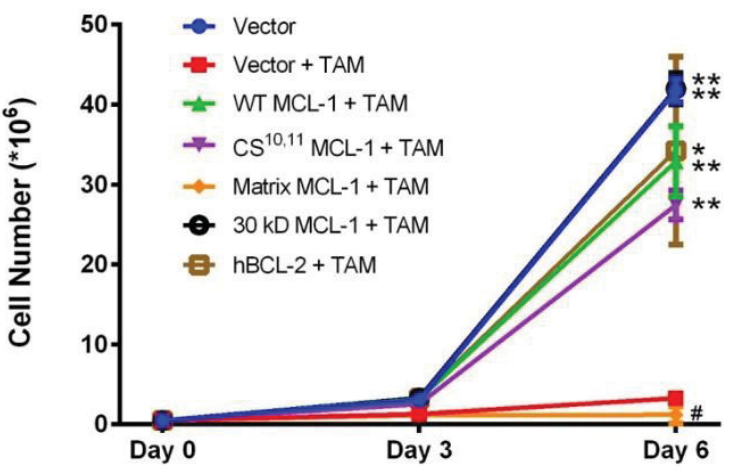

Figure 3-11. Put back MCL-1 mutants for proliferation rescue

(A) Western blot of $\mathrm{Mcl}-\mathrm{I}^{\text {flox/flox }} \mathrm{CRE}^{\mathrm{ERT} 2}$ ESCs overexpressing MIG Vector with or without tamoxifen, WT MCL-1, CS ${ }^{10,11}$, Matrix, $30 \mathrm{kD}$ MCL-1, and hBCL2 with tamoxifen for either 3 days or 6 days. (B) $M c l-1^{\text {flox/flox }} \mathrm{CRE}^{\mathrm{ERT} 2}$ ESCs overexpressing MIG Vector with or without tamoxifen, WT MCL-1, CS ${ }^{10,11}$, Matrix, $30 \mathrm{kD}$ MCL-1, and hBCL2 with tamoxifen were seeded equal number of cells and growth curves were plotted. Bars represent the average of 3 independent experiments and the error bars denote the SEM. $\# p>0.05, * p<0.05$, and $* * p<0.01$ as determined by two-tailed Student $t$ test when compared to $\mathrm{Mcl}-\mathrm{I}^{\text {flox/flox }} \mathrm{CRE}^{\text {ERT2 }}$ ESCs overexpressing Vector treated with tamoxifen. 
overexpressing MIG vector in the absence of tamoxifen showed exponential growth, while the same cells treated with tamoxifen failed to proliferate (Figure 3-11B). All mutants with anti-apoptotic function, WT, CS ${ }^{10,11}, 30 \mathrm{kD}$ MCL-1 and hBCL-2, rescued the growth of the $\mathrm{Mcl}$-1-deleted MIG vector overexpressing ESCs. Matrix MCL-1, without anti-apoptotic activity, failed to rescue the proliferation (Figure 3-11B). These data, taken together, suggest that anti-apoptotic function of MCL-1 is required for ESCs survival and proliferation.

To examine whether different MCL-1 species can rescue the differentiation defect, one million of each ESCs were injected into the flank of nude mice and either tamoxifen or vehicle was performed one week after implantation, five days in a row. $\mathrm{Mcl}$ $1^{\text {flox/flox }} \mathrm{CRE}^{\text {ERT2 }}$ ESCs overexpressing MIG vector treated with vehicle gave rise to big size teratomas 35 days after the injection (row 1), while the same cells treated with tamoxifen failed to generate any tumors during the same period (not shown)

(Figure 3-12A). All three immunodeficient recipients receiving tamoxifen treated $\mathrm{Mcl}$ $1^{\text {flox/flox }}$ CRE $^{\text {ERT2 }}$ ESCs overexpressing MIG WT MCL-1 had big size teratomas (row 2), however, adding back the $30 \mathrm{kD}$ MCL-1 mutant failed to give rise to any size teratomas within the same time frame (data not shown) (Figure 3-12A). $M c l-1^{\text {flox/flox }} \mathrm{CRE}^{\mathrm{ERT} 2} \mathrm{ESCs}$ overexpressing anti-apoptotic only mutant $\mathrm{CS}^{10,11} \mathrm{MCL}-1$ or hBCL2 treated with tamoxifen generated smaller teratomas with pale color (rows 3 and 5). When the Matrix form was added back only one out of three gave rise to pale teratoma 35 days after implantation (row 4) (Figure 3-12A). According to histology review, teratomas from all recipients receiving $\mathrm{Mcl}-\mathrm{I}^{\text {flox/flox }} \mathrm{CRE}^{\mathrm{ERT} 2} \mathrm{ESCs}$ overexpressing the MIG vector treated with vehicle and overexpressing the WT MCL-1 treated with tamoxifen had all three germ layers, including neuroectoderm tissues, surface ectoderm epidermal elements, endoderm tissues like ciliated respiratory epithelium and mesoderm tissues such as striated muscles as well as cartilages. However, teratomas harvested from recipients transplanted with $\mathrm{Mcl}-1^{\text {flox/flox }} \mathrm{CRE}^{\text {ERT2 }}$ ESCs overexpressing the anti-apoptotic only mutant $\mathrm{CS}^{10,11}$ of MCL-1 treated with tamoxifen failed to give rise to distinct three germ layer tissues, but contained foci suggestive of vast amount of primitive undifferentiated tissues. PCR for deleted alleles from teratomas showed distinct deletion bands in all samples treated with tamoxifen (Figure 3-12B). And further immunoblotting from teratoma samples exhibited good deletion efficiency and overexpression levels except that one teratoma sample harvested from the recipient receiving tamoxifen treated $\mathrm{Mcl}$ $I^{\text {flox/flox }} \mathrm{CRE}^{\mathrm{ERT} 2}$ ESCs overexpressing Matrix showed observable endogenous MCL-1 bands (Figure 3-12C). These data, taken together, indicate that exogenous WT MCL-1 can rescue both the proliferation and differentiation of $M c l-1$-deleted ESCs, however, anti-apoptotic only mutants, $\mathrm{CS}^{10,11}, 30 \mathrm{kD}$ MCL-1 and hBCL-2, could only rescue proliferation. Neither anti-apoptotic only mutants nor Matrix species of MCL-1 were able to fully rescue the differentiation in the Mcl-1-deficient ESCs.

\section{Zygote Injection to Generate MCL-1 Mutant Mice}

To study MCL-1 mutants (CS ${ }^{10,11}$ and $30 \mathrm{kD}$ MCL-1) in a more physiological setting, we performed zygote injection to generate mutants knock-in mice. 
A

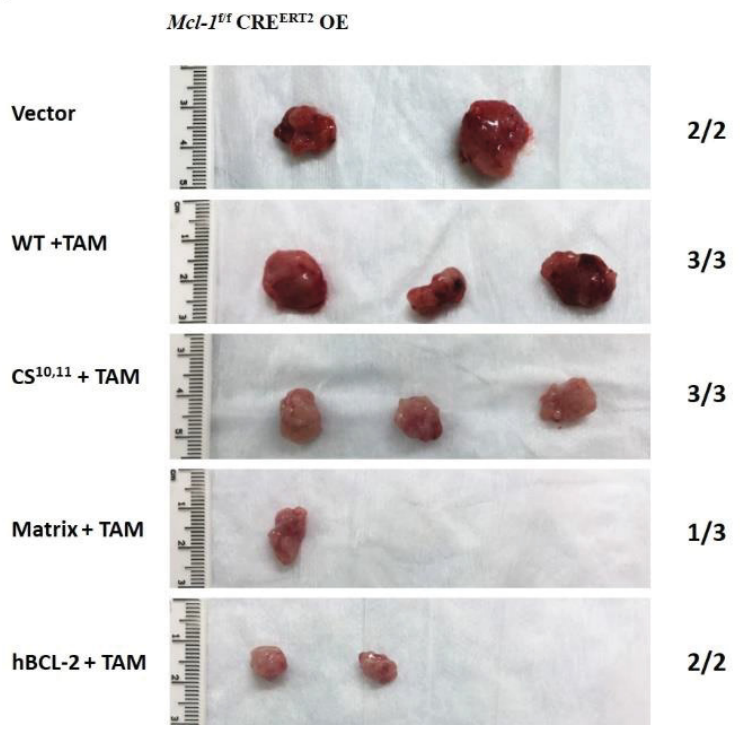

B

C

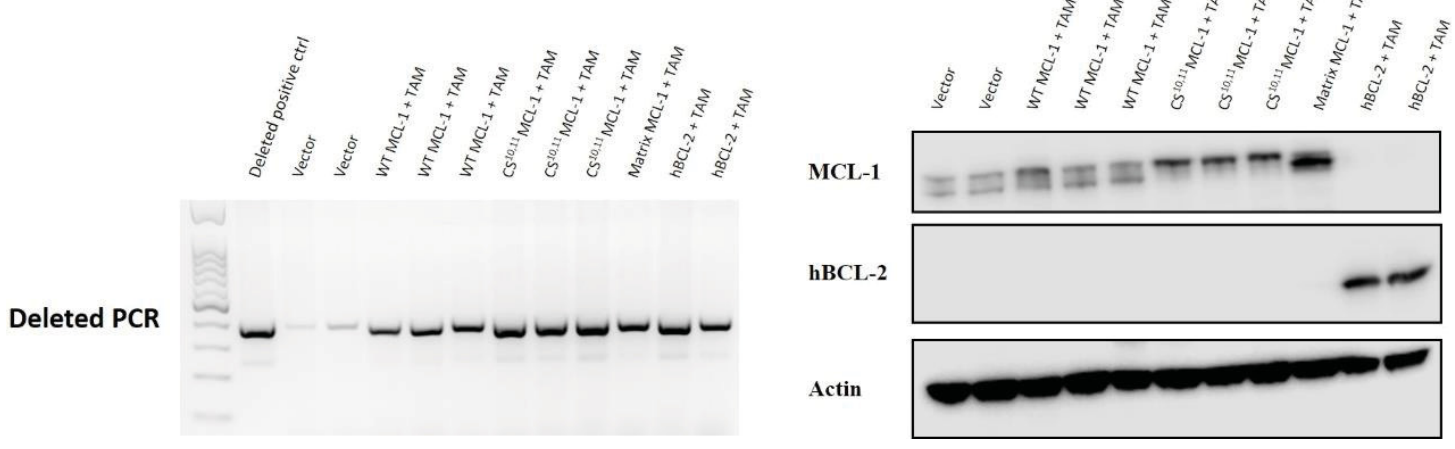

Figure 3-12. Teratoma formation needs both forms of MCL-1

(A) One million $\mathrm{Mcl}-\mathrm{f}^{\text {flox/flox }} \mathrm{CRE}^{\mathrm{ERT2}} \mathrm{ESCs}$ overexpressing either MIG Vector, WT MCL-1, CS ${ }^{10,11}$, Matrix, $30 \mathrm{kD}$ MCL-1, or hBCL2 ESCs were injected into the flank of immunodeficient recipient and tamoxifen gavage was performed one week after the implantation, five days in a row. Tumors were palpated every the other day and all the tumors were harvested 35 days post implantation. (B) DNA was extracted from each teratoma lysate and PCR was performed for the deleted allele. (C) Immunoblots from each teratoma lysates to show endogenous MCL-1 deletion as well as the exogenous mutant overexpression. 
In vitro transcribed Cas9 mRNA, guide RNA (F3 or R2), and guide RNA related ssODN were injected into mouse zygotes. The ssODNs consisted of either symmetric or asymmetric homologous arms, $\mathrm{Mcl}-1$ amino acid 10, 11 mutagenesis, and silent mutation of CRISPR recognition sites in order to prevent recurrent targeting. The asymmetric repair template design has been suggested to increase the homologous recombination efficiency [111]. According to the deep sequencing result of 19 founders' toe DNAs from zygote injection, 11 of them had mutations, with either deletion (red bar), insertion (green bar) or replacement mutation (orange color). The total mutation efficiency in founders was $58 \%$ and $31.5 \%, 10.5 \%$ and $16 \%$ for deletion, insertion and replacement mutation, respectively (Figure 3-13). Two founders, xy007 and xy009, obtained the desired mutagenesis encoding amino acids 10, 11 aspartic acid, aspartic acid to replace the original wild-type isoleucine, glycine with mutation percentages $3.34 \%$ and $14.99 \%$, respectively. Interestingly, xy012 founder carried a desired $\mathrm{CS}^{10,11}$ mutation followed by an in-frame stop codon TAG, which presumably would generate $30 \mathrm{kD}$ KI mice with a mutated allele frequency of $21.88 \%$ (Figure 3-13). Since Cas9 is active as the zygotes divide, the founders are expected to be germline mosaic and the percentages roughly represent the mutated allele frequencies (Figure 3-13). We bred the desired $\mathrm{CS}^{10,11} \mathrm{KI}$ founders xy007 and xy009 as well as the KI plus stop codon mouse, presumably $30 \mathrm{kD}$ $\mathrm{KI}$ founder, in the same mouse background C57BL/6J to generate KI F1 generation.

Genomic DNAs extracted from tails of KI F1 pups were amplified and subjected to Taq I enzyme digestion (Figure 3-14A) and Sanger sequencing (Figure 3-14B). CS ${ }^{10}$, ${ }^{11}$ mutagenesis creates a unique Taq I enzyme site, therefore, KI alleles could be detected by Taq I cleavage. Compared with uncut, full-length amplicon plus cleavage bands suggested KI heterozygous in the presence of Taq I (Figure 3-14A). Wild-type from Sanger sequencing were determined by comparison to mouse $\mathrm{Mcl}-1$ genomic sequences and heterozygous mutations were determined by filtering out the known sequence of the WT allele (Figure 3-14B). CS ${ }^{10,11} \mathrm{KI} F 1$ pups (WT/ $\mathrm{CS}^{10,11}$ heterozygous) were successfully obtained from xy009 founders but not xy007. The reason could be that the mutated allele frequency in xy007 was too low to get germline transmission. $\mathrm{CS}^{10,11} \mathrm{KI}$ followed by an in-frame stop codon F1 pups (WT/ $\mathrm{CS}^{10,11} \mathrm{TAG}$ ) were also derived. To dilute out potential off-target effects from CRISPR-Cas9, derived heterozygous F1 CS ${ }^{10}$, ${ }^{11} \mathrm{KI}$ and $\mathrm{CS}^{10,11}$ TAG were bred back to WT C57BL/6J mice for at least 3 generations. One-time $\mathrm{CS}^{10,11} \mathrm{KI}$ F1 heterozygous crossbreed generated seven pups. Genomic DNAs collected from neonatal tails were amplified and treated by Taq I enzyme digestion and cleavage bands only in the presence of Taq I suggested homozygous KI pups

(Figure 3-14C). Homozygous mutations were further validated by Sanger sequencing and compared to mouse $\mathrm{Mcl}-1$ genomic sequences (Figure 3-14D).

Immunoblotting of neonatal tail fibroblast cells from the seven pups generated by $\mathrm{CS}^{10,11} \mathrm{KI} F 1$ heterozygous mice showed no obvious lower band (Matrix isoform) of MCL-1 in Taq I and Sanger sequencing validated CS ${ }^{10,11} \mathrm{KI}$ homozygous (\#1 and \#7). Heterozygous (\#2, \#4, \#5, and \#6) exhibited decreased lower bands of MCL-1 compared with WT littermate (\#3) (Figure 3-15A). Subcellular localization suggested $\mathrm{CS}^{10,11}$ 


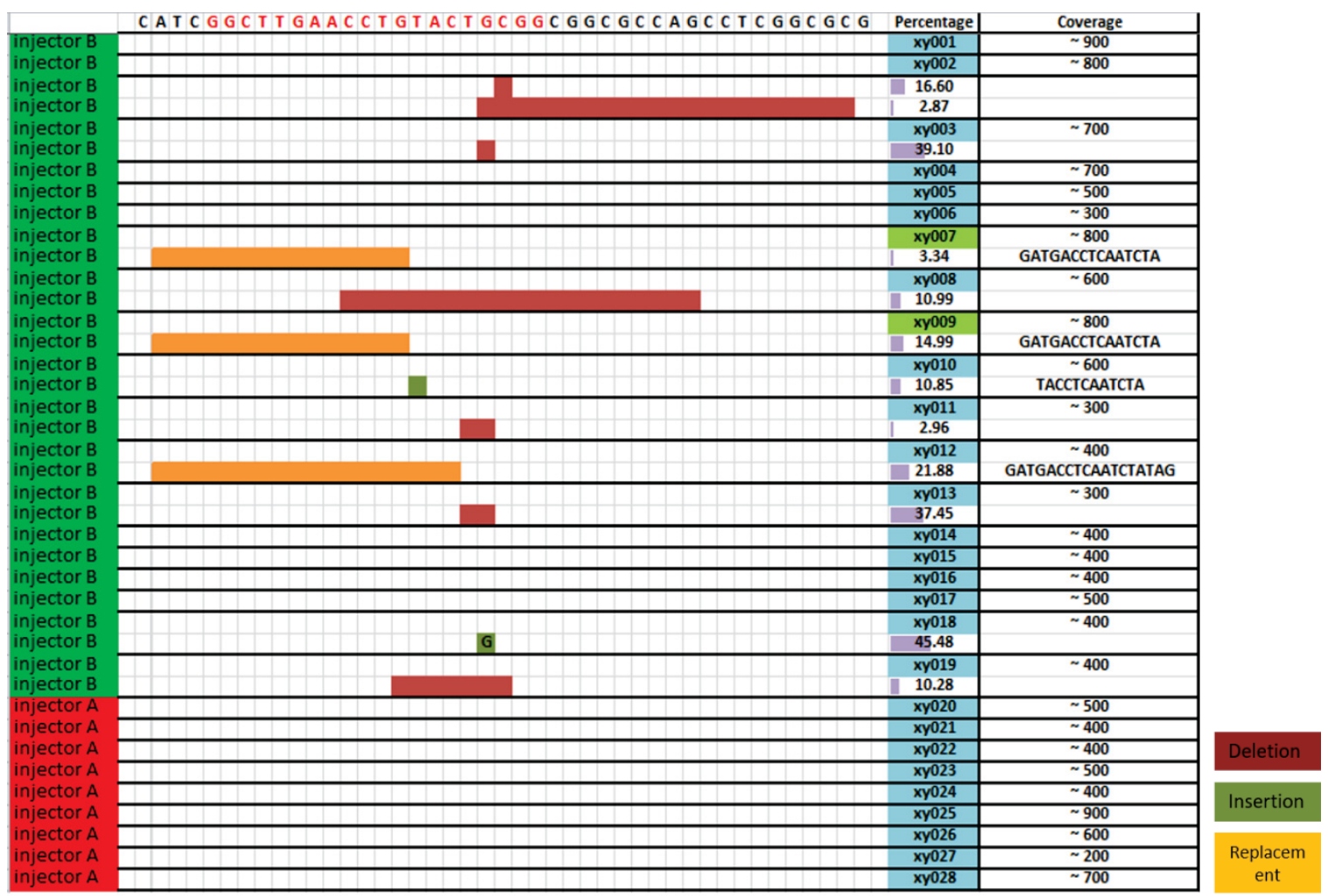

Figure 3-13. Deep sequencing shows mutations and mutation efficiencies in founders after zygote injection

(A) DNA was extracted from the toe of each founder mouse generated from zygote injection. Amplicons from different samples were pooled together and barcoded by another round of PCR by using a Nextera Index Kit. The library prepared from the final PCR products of indexed samples was subjected to a $300 \mathrm{bp}$-paired-end sequencing with Illumina Miseq for the targeted region. Red, green, and orange bars suggest deletion, insertion, and replacement mutation, respectively. Percentages represent the mutated allele frequencies. 
Figure 3-14. Derivation of $\mathrm{CS}^{10,11}$ and $30 \mathrm{kD} \mathrm{KI}$ mice

(A) DNAs extracted from tails of KI F1 pups were amplified and subjected to Taq I enzyme digestion. (B) DNAs extracted from tails of KI F1 pups were amplified and sent for Sanger sequencing. (C) Genomic DNAs were extracted from the toes of the pups derived from $\mathrm{CS}^{10,11} \mathrm{KI}$ F1 heterozygous crossbreeding. Then, DNA collected were amplified and treated by Taq I enzyme digestion. (D) Amplicons collected were sent for Sanger sequencing for further validation. 
A
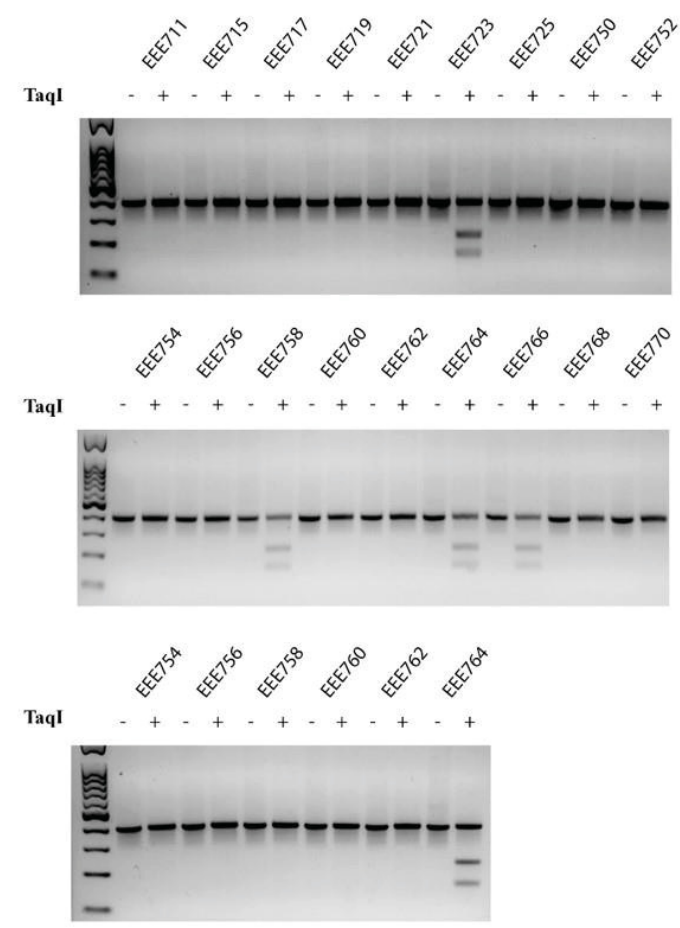

C

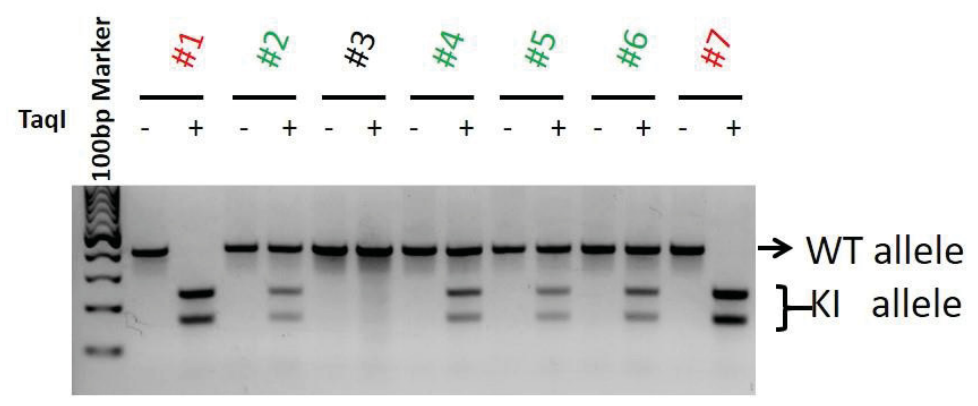

Homozygous: \#1, \#7

Heterozygous: \#2, \#4, \#5, \#6

Wildtype: \#3
B

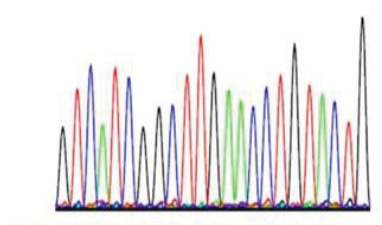

WT: GTCATCGGCTTGAACCTGTAC

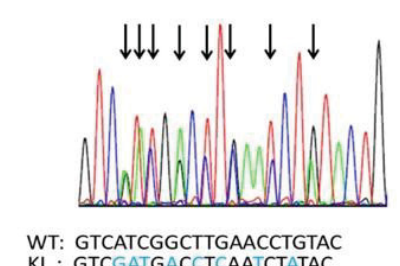

$\mathrm{KI}$ : GTCGATGACCTCAATCTATAC

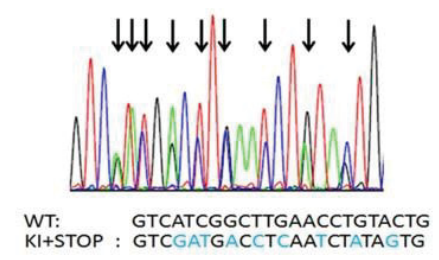

D
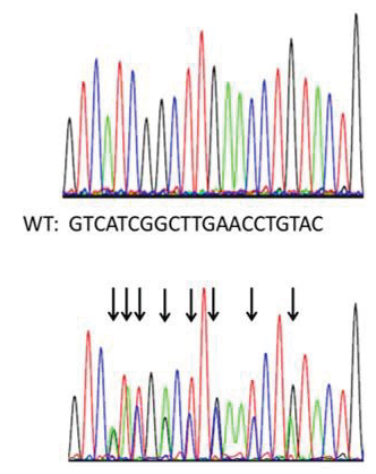

WT: GTCATCGGCTTGAACCTGTAC KI : GTCGATGACCTCAATCTATAC

Taql site

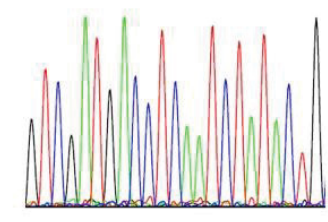

KI : GTCGATGACCTCAATCTATAC Taql site 
A

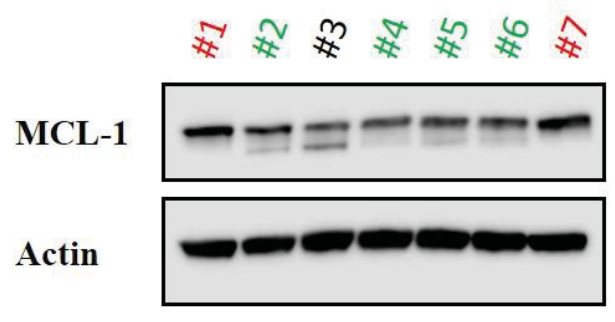

Homozygous: \#1, \#7

Heterozygous: \#2, \#4, \#5, \#6 Wildtype: \#3
B

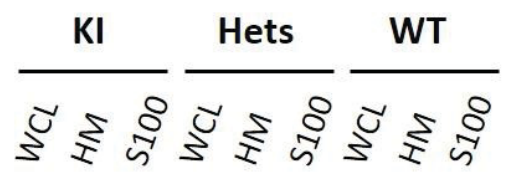

MCL-1

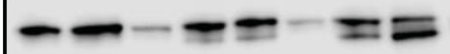

OPA-1

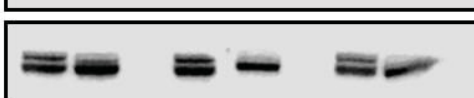

BCL-2

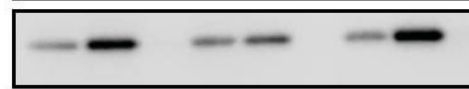

BAX

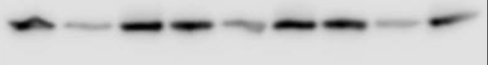

C

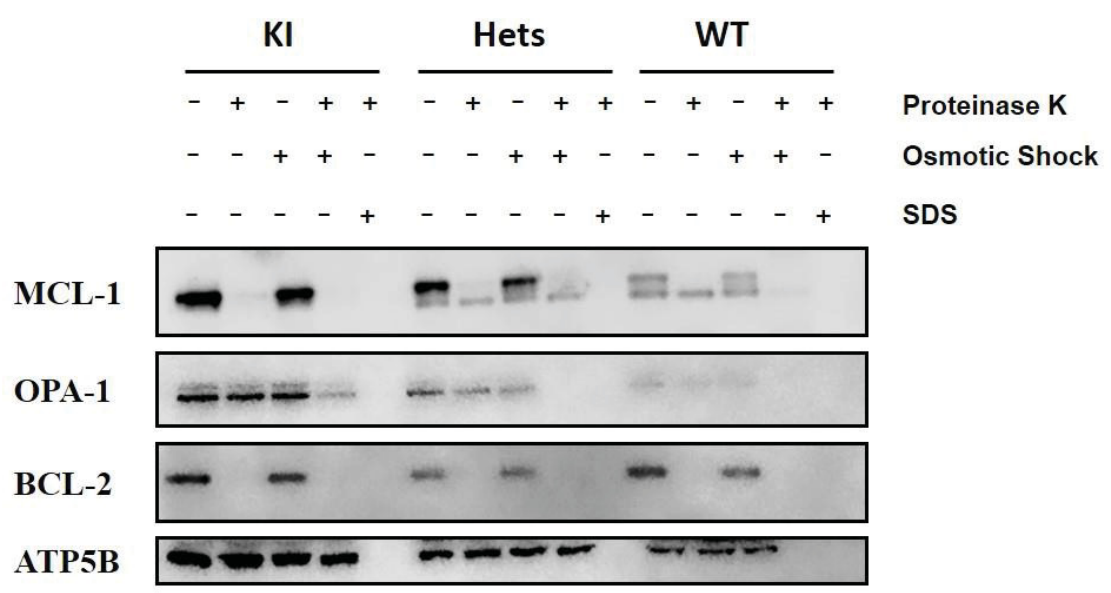

Figure 3-15. Validation of $\mathrm{CS}^{\mathbf{1 0}, 11}$ and $30 \mathrm{kD}$ KI mice

(A) Western blot of the neonatal tail fibroblast cells from the WT, $\mathrm{CS}^{10,11} \mathrm{KI}$

homozygous and $\mathrm{CS}^{10,11}$ heterozygous validated from both Taq I and Sanger sequencing. $\# 1$ and $\# 7$ are $\mathrm{CS}^{10,11} \mathrm{KI}$ homozygous, \#2, \#4, \#5, and \#6 are heterozygous, and \#3 is WT littermate. (B) Mitochondria enriched, heavy membrane (HM) or cytosol (S100) fractions were isolated from $\mathrm{CS}^{10,11} \mathrm{KI}$ homozygous, heterozygous, and WT neonatal tail fibroblast cells. Whole cell lysates (WCL) acted as a control. Fractions were resolved and immunoblotted for MCL-1. OPA-1, BCL-2 or BAX worked as intermitochondrial membrane, outer membrane or cytosolic marker, respectively. (C) Mitochondria isolated from $\mathrm{CS}^{10,11} \mathrm{KI}$ homozygous, heterozygous, and WT neonatal tail fibroblast cells were subjected to proteinase $\mathrm{K}(\mathrm{PK})$ proteolysis. Osmotic shock was used to disrupt outer mitochondrial membrane (OMM), and detergent SDS was used to disrupt both inner and outer mitochondrial membranes. Lysates were collected and subjected to immunoblot analyses for MCL-1, OPA-1 (intermembrane marker), BCL-2 (outer membrane marker), and ATP5B (matrix marker). 
MCL-1 enriched in the heavy membrane (HM) as WT MCL-1 (Figure 3-15B). Further proteinase $\mathrm{K}$ assay indicated that WT MCL-1 retained dual localization as shown by proteinase K sensitive upper band and resistant lower band (Figure 3-15C). CS ${ }^{10,11} \mathrm{KI}$ MCL-1 only exhibited one band and was completely sensitive to enzyme digestion, suggesting its outer mitochondrial only localization (Figure 3-15C). 


\section{CHAPTER 4. DISCUSSION}

\section{MCL-1 in Normal Biology}

Gene-ablation study uncovered a non-redundant and distinct role of MCL-1 as a BCL-2 family member. Deletion of $\mathrm{Mcl}-1$ resulted in embryonic lethality due to periimplantation failure on E3.5 [16], however, genetic deletion of other anti-apoptotic BCL2 family members do not result in such severe phenotype [11-14]. Induced deletion of Mcl-1 during different stages of hematopoiesis and different lineages indicates that MCL1 is essential for the survival and differentiation for multiple hematopoietic lineages, including pre-B and progenitor T cells, HSCs, CMPs, CLPs, as well as mature neutrophils, plasma cells, red blood cells, and NK cells, etc. [17-23]. In contrast, other pro-survival family members play much more selective roles in hematopoiesis $[11,14$, 24, 25]. Additionally, cardiac-specific ablation of $\mathrm{Mcl}-1$ also leads to a dilated cardiomyopathy and rapidly fatal phenotype [26, 27]. However, all previous gene ablation studies disable both classical anti-apoptotic activity on the outer mitochondrial membrane and mitochondrial function species in the mitochondria matrix. Therefore, to determine the relative contribution of MCL-1's different functional roles for supporting the survival and differentiation of various cellular lineages, it is important to dissect the two species. It is proposed that both species may be critical for normal cell biology, including supporting survival, promoting proliferation and differentiation, as well as regulating the homeostasis response to growth factors or cellular signals. However, these two species may contribute differently during developmental stages or on distinct cell types. For example, hematopoietic cells are highly proliferative and more vulnerable to cellular stress, therefore, they are extremely dependent on anti-apoptotic MCL-1 for survival. Another example are pluripotent stem cells which remain relatively metabolic inactive by utilizing anaerobic glycolysis. When differentiating into their progenies, metabolic demands from a major glycolysis to oxidative phosphorylation switch of those pluripotent stem cells may require mitochondrial function of MCL-1.

\section{MCL-1 in Embryonic Development}

The study that germline ablation of $\mathrm{Mcl}-1$ in mice resulted in an early periimplantation failure at E3.5 highlights a non-redundant and distinct role of MCL-1 among BCL-2 family members [16]. Other pro-survival members do not have such an early developmental defect, suggesting that MCL-1 may have a unique function during this early embryonic developmental stage. In the $\mathrm{Mcl}-1$ germline ablation study, the authors found deletion of $\mathrm{Mcl}-1$ resulted in early embryonic lethality, however, they claimed the defect was a trophectoderm (TE) defect rather than the inner cell mass (ICM) apoptosis. This was demonstrated by the evidences that $\mathrm{Mcl}-1$-null blastocysts failed to attach in vitro and $\mathrm{Mcl}-1$ null blastocysts show no signs of increased apoptosis, but a delay in maturation beyond the pre-compaction stage, further indicating a role of MCL-1 beyond anti-apoptosis during early embryonic stage [16]. 
In our study, we designed nine guide RNAs to target $\mathrm{Mcl}-1$ 's start codon and total mutation efficiencies range from 75\%-96\%. Unlike SV40-transformed MEFs which can tolerate MCL-1 loss, disruption of Mcl-1's first methionine in ESCs all generated a $30 \mathrm{kD}$ short truncated protein of MCL-1 maintaining its anti-apoptotic core, suggesting that MCL-1's anti-apoptotic function is essential for ESC survival. Although $30 \mathrm{kD}$ MCL-1 maintained classical anti-apoptotic function, mutant ESCs presented sick phenotypes and failed to differentiate to extraembryonic endoderm, which is consistent with the trophectoderm defect from genetic ablation of Mcl-1. Further q-PCR results even suggested broad differentiation defects, including ectoderm, mesoderm, and endoderm layers. Rescue experiment by putting back different MCL-1 mutant showed only WT MCL-1 can fully rescue the differentiation defect of teratoma formation, neither antiapoptotic $30 \mathrm{kD}, \mathrm{CS}^{10,11} \mathrm{MCL}-1$ nor mitochondria matrix species completely rescue the defect. These data, taken together, suggest that in addition to MCL-1's required antagonism of cell death by the C-terminal region, MCL-1's N-terminus is required for efficient cellular differentiation, potentially by facilitating MCL-1's import into the mitochondrial matrix. However, the mechanistic underpinnings of the anti-apoptotic function and mitochondrial promoting activity in determining ESC differentiation remains to be elucidated.

Metabolic Requirements during Embryogenesis. Mitochondria of the oocyte and early embryo are known to be small with few cristae and less electron transport chains, but those mitochondria are highly active in oxidative phosphorylation to generate ATP in support of the metabolic demands of early development [112-114]. And it is wellknown that mitochondrial replication of the oocyte is arrested in metaphase II of meiosis and is only reactivated upon implantation into the uterine wall [115]. Post-fertilization, mitochondria undergo dramatic maturation into the elongated "spaghetti-like" and cristae-rich structures and failure to acquire this mature mitochondrial ultrastructure results in arrested embryos [116-118]. Further following implantation, a transition from glycolysis to oxidative phosphorylation is essential for successful early embryogenesis, as shown by a developmental delay or late onset lethality with mutations that impair oxidative metabolism $[119,120]$. MCL-1 is required for early implantation, however the mechanism has still remained elusive. It is possible that MCL-1 facilitates mitochondrial ultrastructure and provides sufficient energy production that is required for the trophectoderm to implant. Another possibility is that MCL-1 promotes mitochondrial physiology and oxidative phosphorylation to prime the differentiation of cytotrophoblast cells, failure of which results in a defective trophectoderm to implant. Therefore, it is conceivable that other than MCL-1's required antagonism of cell death, MCL-1's role to maintain normal mitochondrial physiology and promote differentiation is also important during early embryonic development.

Metabolic Determinants of Embryonic Stem Cell Fate. Embryonic stem cells are derived from the inner cell mass of the pre-implantation blastocyst around E3.5 and represent the gold-standard of "stemness", which means they obtain pluripotency, capable of differentiating into broad tissue-specific lineages, and self-renewal, the ability 
to self-replicate maintaining the undifferentiated pool. Similar to their tissue origin, embryonic stem cells are characterized by a low mtDNA content and immature mitochondrial ultrastructure with poorly developed cristae, which is also a universal feature across a variety of stem cell populations, including hematopoietic stem cells (HSCs) and mesenchymal stem cells (MSCs) [121-123]. It is now well-accepted that glycolysis fuels not only energy (ATP) but also building blocks (carbon, nitrogen, and hydrogen) for stem cell proliferation and self-renewal [124, 125]. Stimulating glycolysis in pluripotent stem cells through hypoxia or suppressing mitochondrial respiration promotes "stemness" while inhibition of glycolysis halts proliferation and accelerates cell death [126-128].

Pluripotent stem cells and their progenies have very different energetic requirement. Differentiated cells no longer sustain high proliferation rate, but rather perform specific specialized functions, such as rhythmic contraction in cardiomyocytes or electrical impulses in neurons, therefore, they demand large amount of energy to fuel those processes rather than anabolic synthesis for rapid proliferation [129]. When induced to differentiate, specific energetic and metabolic capacities are required to overcome barriers of the state conversion, evidenced by the downregulation of glycolysis and stemness genes while stimulation of mtDNA replication, mitochondrial biogenesis, maturation of extensive, interconnected networks of elongated and cristae-rich mitochondria as well as upregulation of TCA cycle players and electron transport chain components [123, 130-132]. Thus, a metabolic switch from glycolysis to mitochondrial oxidative phosphorylation is essential for stem cell differentiation; however, the mechanism of a causal role in cell fate determination remain to be unknown.

Several studies have emphasized that mitochondrial regulation of reactive oxygen species (ROS) as well as stem cell differentiation inducers and antioxidants, impair the differentiation capability of HSCs and MSCs [132-135]. Other researchers report that several signaling pathways have been involved in regulating the metabolic switch. It is well-recognized that Smad1 and STAT3 act as key components to support stemness of pluripotent stem cells through signaling pathways mediated by bone morphogenetic protein (BMP) and leukemia inhibitory factor (LIF), respectively [136, 137]. PI3KmTOR pathway has also been revealed by several studies to regulate stemness of HSCs and tuberous sclerosis complex 1 (TSC1) activity, an upstream negative regulator of mTOR, is required to support HSC stemness by repressing mitochondrial biogenesis and ROS production [138-140]. Other than the metabolic regulation, cellular signaling, epigenetic control of transcription programs has also been reported to determine the stem cell fate. One example is that Moussaieff et al. showed that glycolysis-derived acetylCoA prevents histone deacetylation and blocks differentiation of ESCs [141].

In our work, we showed that N-terminal truncated mutant of MCL-1 ESCs failed to differentiate to broad germ lineages, including extraembryonic endoderm, ectoderm, mesoderm, and endoderm. N-terminal sequences of MCL-1 contain mitochondria targeting sequences, deletion of which abrogates MCL-1's importation into the mitochondrial matrix. Matrix localized MCL-1 has been suggested to support mitochondrial ultrastructure, promote mitochondrial fusion and fission, and facilitate the 
assembly of ATP synthase oligomers and oxidative phosphorylation [38]. It is possible that in the absence of matrix species of MCL-1, ESCs cannot make the switch from glycolysis to oxidative phosphorylation, and therefore, fail to overcome the energetic and metabolic barriers to undergo differentiation. Another possibility is that the N-terminus of MCL-1 is required for binding to certain key factors to activate differentiation pathways or inhibit signaling that maintain pluripotency. It is suggested that MCL-1 interacts with STAT3 at the window of implantation to promote the mesenchymal to epithelial transition (MET) required for successful implantation [142, 143].

\section{MCL-1 in Cardiac Homeostasis}

Mitochondrial Function in Cardiomyocyte Development. The development of the heart starts around E7.5, beating heart tube may be observed at E8.5, and the primary four cardiac chambers are formed around E10.5 [144]. Cardiomyocyte development has also been suggested to be dependent on mitochondrial status as cardiomyocytes from early embryos around E9.5 which show sparse fragmented mitochondria with poorly formed cristae and immature ultrastructure. Those immature mitochondria undergo extensive maturation into elongated and branched mitochondria with abundant and organized cristae by E13.5 [133]. Studies have shown that early induction of mitochondrial maturation accelerates cardiomyocyte differentiation, while forced arrest in the immature status of mitochondria impairs the differentiation of cardiomyocytes $[133,145]$. MCL-1 is essential for cardiac development as evidenced by our previous study that induced deletion of $\mathrm{Mcl}-1$ in heart by $\mathrm{Ckmm}$-CRE at E13.5 leads to a fatal dilated cardiomyopathy [26].

MCL-1's Dual Functions in Heart Homeostasis. Cardiomyocytes are permanent cells and they lose their regenerative ability after postnatal day 7; therefore, they can only be repaired by connective tissues through fibrosis [146]. Deletion of $\mathrm{Mcl}-1$ in adult mice heart by tamoxifen inducible $M y h-\mathrm{CRE}^{\mathrm{ER}}$ also results in a rapid, fatal cardiomyopathy. However, low but significant levels of apoptosis was observed, which is consistent with previous studies showing that even low levels of cell death are sufficient to cause cardiomyopathy because they lack of regeneration capability [26, 147]. Further genetic ablation of pro-apoptotic Bax and Bak rescue the survival of cardiac-specific $\mathrm{Mcl}-1$ deletion and improves cardiac function, indicating that MCL-1 prevents heart failure and overt cardiomyopathy by blocking cell death [26]. Although deletion of Bax and Bak rescues loss of Mcl-1 and those mice did not display significant cardiac dysfunction, further analyses of the hearts from those mice reveal the heterogeneous myocardial organization with rounded and disorganized mitochondria. Respiratory defect as well as decreased mtDNA levels could not be rescued by Bax and Bak deficiencies [26]. Additionally, cardiac-specific $M c l-1$ deletion in the absence of $B a x$ and Bak shows cardiac dilation and contractile dysfunction under the isoproterenol challenge [26]. Taken together, these data suggest that both anti-apoptotic species and 
mitochondria matrix species are necessary for heart homeostasis by promoting cardiomyocytes survival and facilitating mitochondrial physiology, respectively.

\section{MCL-1 in Hematopoiesis}

Previous specific genetic ablation studies during different stages of hematopoiesis revealed that MCL-1 is unique and required for development and maintenance of hematopoiesis [17-23]. Deletion of MCL-1 in early hematopoietic progenitors, HSCs, double-negative $\mathrm{T}$ cells and pro-B stages, leads to an increased apoptosis and arrested development $[17,19]$. In contrast, other pro-survival BCL-2 family members seem to play much more selective roles limited to more mature cells. Genetic ablation of BCL-2 and BCL- $\mathrm{X}_{\mathrm{L}}$ leads to defects of mature lymphocytes and red blood cells, respectively [11, 24]. A1 isoforms play a role, but are not indispensable for granulocyte and lymphoid development $[14,25]$. Similar to embryonic stem cells discussed earlier, an energetic and metabolic reprogramming from glycolysis to oxidative phosphorylation is a universal feature across a variety of stem cell populations and is also essential for hematopoietic lineage specification [121]. Therefore, it is possible that both the Matrix and antiapoptotic MCL-1 forms are required to provide metabolic demands for hematopoietic lineage differentiation and to antagonize cell death to sustain maintenance of progenitor pool, respectively.

More committed lineages, such as mature lymphocytes or myelocytes, have different metabolic demands and no longer sustain high proliferation rate as their progenitors, but rather perform immune surveillance or phagocytosis under the regulation of cytokines and growth factors [6, 148]. Deletion of MCL-1 in mature hematopoietic lineages, such as mature neutrophils, plasma cells, red blood cells, and NK cells, have also been reported to cause dramatic increased apoptosis, indicating that more committed lineages are highly regulated by apoptosis [18, 20-23]. It is conceivable that matrix localized MCL-1 may be important for early progenitors as it facilitates the mitochondrial physiology to prime stem cells for differentiation, while anti-apoptotic MCL-1 may be more important for survival of the mature cells. Further studies are needed to separate these two functions of MCL-1 in order to understand their individual contributions to hematopoietic homeostasis.

\section{MCL-1 in Cancer Biology and Cancer Therapy}

MCL-1 has been reported to be overexpressed in many human cancers, including acute lymphoblastic leukemia (ALL), acute myelogenous leukemia (AML), chronic lymphocytic leukemia (CLL), chronic myelogenous leukemia (CML), neuroblastoma, melanoma, breast cancer, lung cancer [31]. The rationale for cancer cells displaying high level of MCL-1 is that those cancer cells often violate important cellular checkpoints which can drive the cells to die through apoptosis. Therefore, cancer cells need strategies for survival under the increased apoptotic stress by either downregulating pro-apoptotic regulators or, more frequently, upregulating pro-survival members like MCL-1 [36]. 
More importantly, elevated level of MCL-1 expression often correlates with disease progression, chemotherapeutic resistance and relapse, highlighting the importance of understanding MCL-1's contributions and regulations [32-35].

\section{Targeting MCL-1 as an Anti-apoptotic Protein}

Since cancer cells are so "addicted" to anti-apoptotic BCL-2 family members as we discussed earlier, researchers have been working on developing small molecules to antagonize those pro-survival members. ABT-737/263, navitoclax, binds specifically with high affinity to pro-survival proteins BCL-2, BCL-xL, and BCL-w, but not to MCL1 and has been reported to induce regression of many solid tumors [94-96]. Though ABT-737 shows promising killing effect in certain tumors, its efficacy as a single agent is limited because of MCL-1 overexpression, which also represents a potential mechanism of resistance $[149,150]$. Furthermore, the dose-limiting severe thrombocytopenia from navitoclax also quenched the enthusiasm for further clinical development of this compound [151]. ABT-199, venetoclax, has been developed and shown to have subnanomolar affinity to BCL-2 with good anti-tumor activity sparing platelets on preclinical studies of many leukemia and lymphomas [152-155]. Most recent clinical trials have identified ABT-199 as a very effective drug either as a single agent or combined with traditional chemotherapy drug on CLL, even in relapsed or refractory patients, non-Hodgkin lymphoma, especially mantle cell lymphoma [156-158].

In 2016, Kotschy et al. developed a small molecule S63845 that specifically binds to the BH3-binding groove of MCL-1 with high affinity and showed very potent antitumor activity with an acceptable safety margin either alone or in combination with other traditional chemotherapy drugs in treating a variety of tumors, such as multiple myelomas, leukemia, lymphoma cells and some other solid tumors [37]. The authors showed that S63845 kills tumor cells in a pro-apoptotic BAX/BAK dependent manner, which highlights MCL-1's anti-apoptotic function as a target in cancer therapeutics [37]. This study provides preclinical evidence that MCL-1 is a promising target. Inhibition of MCL-1 with S63845 is efficacious and tolerable, which may be explained by the overexpression of MCL-1 in tumor cells, making them more vulnerable than normal cells. Further studies are warranted to explore the potential of S63845 for the treatment of human cancers.

\section{Targeting Matrix-MCL-1 for Cancer Therapy}

The function of MCL-1 in facilitating mitochondrial physiology offers another clue for targeting MCL-1 in cancer therapeutics. In contrast to conventional opinions, although mutations affecting mitochondrial functions are common in cancer cells, they do not disrupt the energetic and metabolic functions but rather reprogram the mitochondrial bioenergetics and biosynthetic status to meet the demands of cancer cells [43]. Functional mitochondria are essential for cancer cell survival, and cancer therapies targeting mitochondria have already been shown to be promising for cancer treatment [75]. 
Over the past decade, it has been demonstrated that for various malignancies, including leukemia and various solid tumors, cancer cells are heterogeneous and functionally inequivalent. Studies have shown that despite identical genetic background, there are a subset of cancer cells that acquire "stemness" features and have the ability to self-renew and give rise to heterogeneous cancer population. Thus, such cancer cells have been termed cancer stem cells (CSCs) or tumor-initiating cells (TICs) [159-161]. Similar to the normal stem cells, CSCs have also been shown to adopt primarily aerobic glycolysis over OXPHOS to maintain their "stemness" as well as proliferate [162-164]. The role of MCL-1 in facilitating mitochondrial physiology, especially promoting OXPHOS, may be beneficial to promote cancer stem cell proliferation. Therefore, it is possible that augmenting the function of matrix MCL-1 could be an effective strategy to induce differentiation and deplete the cancer stem cell pool. Further studies are required to separate the two functions of MCL-1 and find out their relative contribution to cancer initiation and maintenance.

\section{Potential Concerns of Targeting MCL-1 in Cancer Therapies}

MCL-1 is essential for the survival and development of various cell types and inhibition of MCL-1 results in massive cell death and tissue dysfunctions, as discussed earlier [16-21, 26]. A previous study on S63854 provides preclinical evidence that MCL1 is a promising therapeutic target and its inhibition is efficacious and tolerable, which may be explained by the MCL-1 dosage effect. Cancer cells are always displaying increased copies of MCL-1 to avoid undergoing apoptosis, therefore, they are more dependent on MCL-1 and more vulnerable than normal cells. However, for many cells and tissues that are highly dependent on MCL-1 for survival and homeostasis, potential adverse side effects will need to be monitored when targeting MCL-1.

Severe thrombocytopenia from ABT-263, navitoclax, is a good example that its target BCL-xL is an essential pro-survival molecule for megakaryocyte survival to produce platelets $[151,165,166]$. Importantly, our data demonstrate that the antiapoptotic character of MCL-1 is required to keep embryonic stem cells surviving. Both anti-apoptotic and Matrix forms are essential for efficient cellular differentiation. Other studies also suggest that MCL-1 is indispensable for cardiac homeostasis, hematopoiesis, and other functions [17-21,26]. Therefore, the potential adverse side effects associated with pharmacological MCL-1 inhibition should be monitored, such as tissue differentiation and regeneration defects, cardiotoxicity, as well as pancytopenia. 


\section{CHAPTER 5. FUTURE DIRECTIONS}

\section{Contributions of Both MCL-1 Species for ESCs Survival and Differentiation}

Previous work from our laboratory has revealed that two forms of MCL-1 possess different functions due to their distinct localization [38]. Outer mitochondrial membrane localized MCL-1 performs the classical anti-apoptotic function by antagonizing BAX and BAK. However, N-terminal proteinase processed matrix localized MCL-1 does not possess anti-apoptotic activity, but plays a role in promoting mitochondrial physiology, including maintaining mitochondrial ultrastructure, facilitating mitochondrial fusion and fission, supporting the assembly of ATP synthase oligomers and oxidative phosphorylation [38]. Our data show that N-terminal truncated $30 \mathrm{kD}$ mutant ESCs, although maintaining MCL-1's anti-apoptotic function to help ESCs to survive, fail to differentiate to broad germ lineages, indicating that function of MCL-1 beyond antagonizing cell death is required for efficient cellular differentiation. Additionally, rescue experiments by putting back different isoforms of MCL-1 showed that only WT MCL-1 is capable of rescuing the differentiation defect of teratoma formation. Neither anti-apoptotic only $\mathrm{CS}^{10,11} \mathrm{MCL}-1$ nor mitochondria Matrix species can completely rescue the differentiation defect, further suggesting both forms of MCL-1 are essential for ESCs survival and differentiation.

\section{Differentiation Rescue Experiments by Enforced Expression of MCL-1 Species}

Our data demonstrate that only WT MCL-1 is capable of rescuing the differentiation defect but neither OMM mutant anti-apoptotic CS ${ }^{10,11} \mathrm{MCL}-1$ nor mitochondria matrix species completely rescues the defect. Our future experiment will be to enforce the expression of both forms of MCL-1 in Mcl-1-deficient ESCs to see if we can get the complete rescue of differentiation. We could first transduce $\mathrm{Mcl}-\mathrm{I}^{\text {flox/flox }}$ $\mathrm{CRE}^{\text {ERT2 }}$ ESCs overexpressing MSCV-IRES-GFP $30 \mathrm{kD}$ or CS ${ }^{10,11} \mathrm{MCL}-1$ with MSCVI-mCherry Matrix MCL-1 and examine whether the $30 \mathrm{kD}$ plus Matrix or $\mathrm{CS}^{10,11}$ plus Matrix MCL-1 ESCs are able to differentiate into multiple germ layers. To avoid multiple rounds of transduction and flow sort, we could generate one construct with both $30 \mathrm{kD}$ and Matrix or $\mathrm{CS}^{10,11}$ and Matrix connected by a $2 \mathrm{~A}$ peptide [167].

Another strategy will be to first overexpress different MCL-1 mutants, $30 \mathrm{kD}$, $\mathrm{CS}^{10,11}$ or Matrix MCL-1, to the F12 ESCs and then performing the CRISPR-Cas9 targeting on the endogenous MCL-1. If both forms of MCL-1 are required for ESCs differentiation, we expect to see that endogenous $30 \mathrm{kD}$ mutant ESCs generated from CRISPR-Cas9 overexpressing Vector, $30 \mathrm{kD}$ or CS ${ }^{10,11} \mathrm{MCL}-1$ will fail to differentiate to any committed lineages. However, ESCs expressing the endogenous $30 \mathrm{kD}$ mutant transduced with either WT or Matrix MCL-1 should be able to undergo efficient differentiation. By examining the design of our previous guide RNAs, crRNA-R1 could be used to target the endogenous MCL-1 but sparing the exogenous MCL-1 mutants because it targets the 5'-UTR of $\mathrm{Mcl}-1$ genomic DNA. 


\section{Determine Mechanistically How Both Forms of MCL-1 Regulate ESCs Differentiation}

\section{Determine Physiological Mitochondrial Status of Mutant ESCs}

Our work indicates both forms of MCL-1 are indispensable for efficient differentiation of ESCs. However, future studies are needed to understand the mechanisms by which OMM and Matrix MCL-1 collaborate to regulate cellular differentiation. It is conceivable that OMM anti-apoptotic MCL-1 supports ESCs survival by antagonizing BAX and BAK. This was suggested by our previous evidences that no clone with complete $\mathrm{Mcl}-1$ deletion survived after CRISPR-Cas9 targeting and $\mathrm{Mcl}-1$ deleted ESCs failed to expand in culture. Matrix MCL-1, however, may promote the metabolic switch from aerobic glycolysis to OXPHOS by facilitating mitochondrial physiology for ESCs to undergo differentiation.

Therefore, it is important to determine the physiological mitochondrial status of WT ESCs comparing $30 \mathrm{kD}$ mutant ESCs and $\mathrm{Mcl}-1^{\text {flox/flox }} \mathrm{CRE}^{\mathrm{ERT} 2} \mathrm{ESCs}$ in the presence or absence of tamoxifen. We could measure ATP production, oxygen consumption, mitochondrial fusion and fission, supercomplexes of electron transport chain (ETC), mtDNA and mitochondrial membrane potential. Previous work from our laboratory suggests Matrix MCL-1 plays a role in maintaining mitochondrial ultrastructure, facilitating mitochondrial fusion and fission, supporting the assembly of ATP synthase oligomers and oxidative phosphorylation. Therefore, we expect to see that $30 \mathrm{kD}$ mutant ESCs or $\mathrm{Mcl}$-1-deleted $\mathrm{Mcl}-1^{\text {flox/flox }} \mathrm{CRE}^{\text {ERT2 }} \mathrm{ESCs}$, lacking Matrix MCL-1, have defective mitochondrial respiration, decreased production of ATP and mtDNA, abnormal mitochondrial ultrastructure as well as the disrupted electron transport chain supercomplexes. Further experiment will be required to re-intrduce different mutants of MCL-1 to see whether OMM anti-apoptotic MCL-1 or Matrix MCL-1 rescues the mitochondrial physiology defects. We expect to see that WT MCL-1 or Matrix MCL-1 corrects the mitochondrial physiology defects of $30 \mathrm{kD}$ mutant ESCs.

\section{Determine Mechanistically How MCL-1 Regulates ESCs Differentiation}

Our preliminary data suggested that when being induced by differentiation, fulllength MCL-1 protein expression was first increased peaking around 24 hours and then decreasing around $30 \%$ at EB day 10 compared with ESCs at the starting point (Figure 5-1A and B). The expression pattern of mRNA levels of $\mathrm{Mcl}-1$ was consistent with its protein expression, showing that $\mathrm{Mcl}-1$ transcription was dramatically induced for the first 24 hours of the differentiation process before the level dropped at the latter time point (Figure 5-1C and D). Dab-2 mRNA level was performed as a positive control, showing that the extraembryonic endoderm marker was induced along the differentiation process (Figure 5-1E and $\mathbf{F}$ ). These data suggest that MCL-1 may be an early response gene under the regulation of either cytokines or signaling pathways during differentiation. 
Figure 5-1. MCL-1 expression is first induced and then decreased during differentiation process

(A) Immunoblotting of F12 WT ESCs and embryoid bodies at different time point along the differentiation for MCL-1, PARP, Caspase 3 and Actin. (B) Relative expression of MCL-1 protein levels along the differentiation time course. (C) Real-time PCR of induction fold change of control for $M c l-1$ mRNA along the differentiation time course. (D) The curve of relative expression of $\mathrm{Mcl}-1 \mathrm{mRNA}$ levels during the differentiation time course. (E) Real-time PCR of induction fold change of control for Dab2 mRNA along the differentiation time course. (F) The curve of relative expression of $D a b 2$ mRNA levels during the differentiation time course. The assay was done in triplicate for each sample. Error bars represent the standard error of the mean. 
A

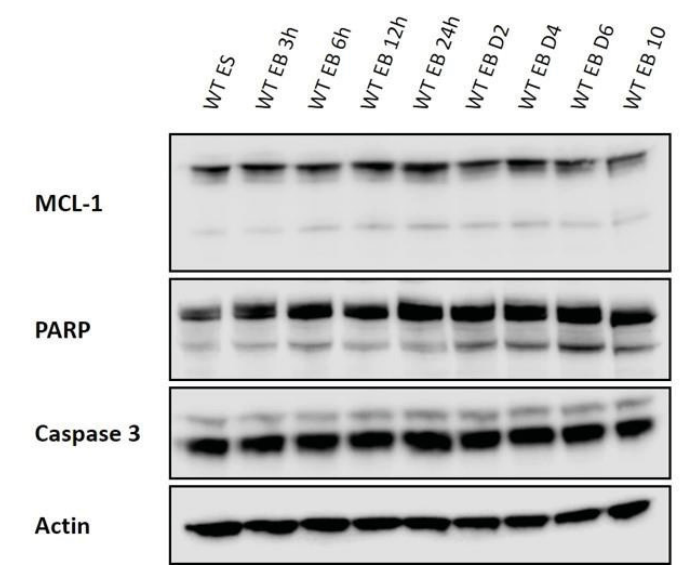

C

Mcl-1

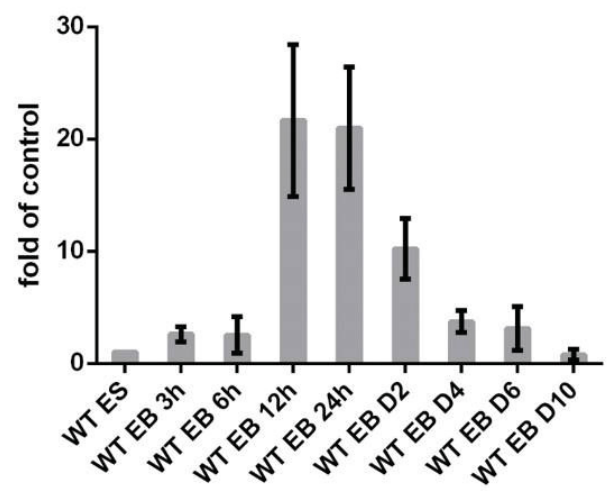

E

Dab2

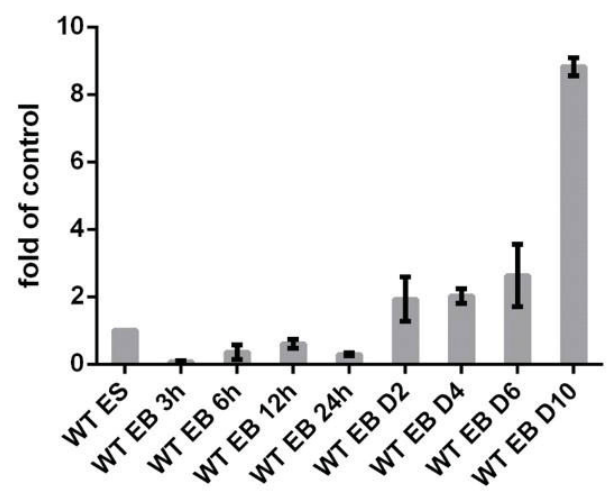

B

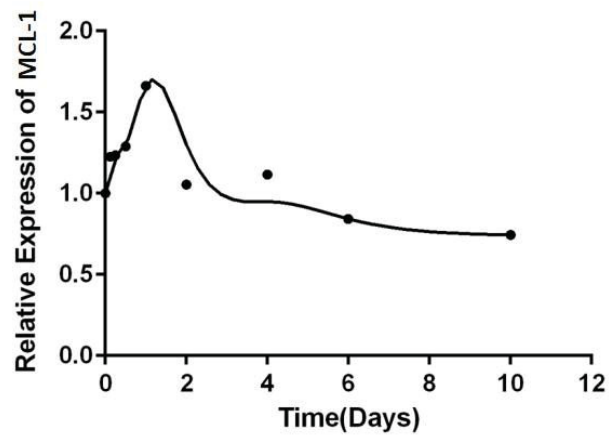

D

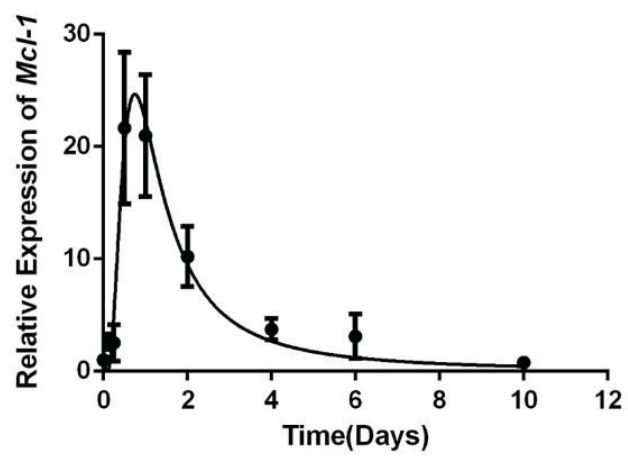

F

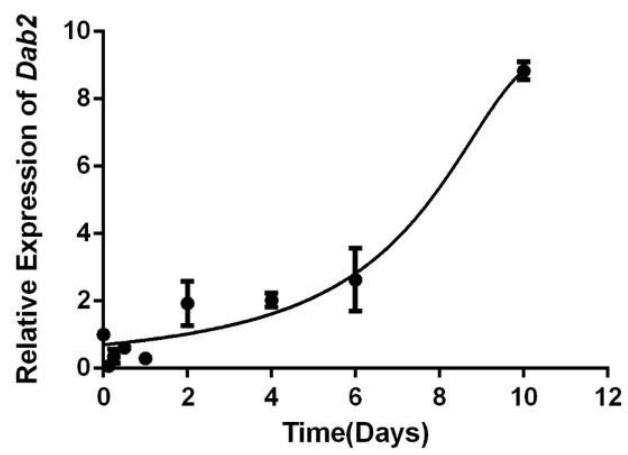


We further performed microarray analysis on $M c l-1^{\text {flox/flox }} \mathrm{CRE}^{\text {ERT2 }} \mathrm{ESCs}$ in the presence or absence of tamoxifen. Principal components analysis (PCA) is a statistical technique for demonstrating the key variables of multidimensional data set. It can be used to simplify the analysis by visualization [168]. Our PCA and corrected PCA data suggested that $\mathrm{Mcl}-\mathrm{I}^{\text {flox/flox }} \mathrm{CRE}^{\mathrm{ERT} 2} \mathrm{ESCs}$ control and $\mathrm{Mcl}$-1-deleted ESCs were very different across gene expression data sets (Figure 5-2A and B). Additionally, our gene set enrichment analysis (GSEA) revealed many potential pathways involved.

Understandably, pathways being correlated with tumorigenesis, progression and invasion have been identified because anti-apoptotic MCL-1 has been suggested to be involved in cancer initiation, progression and relapse as we discussed earlier. Other than the possible anti-apoptotic pathways, there are two top targets identified that may be relevant, SUZ12 and the epithelial to mesenchymal pathway (Figure 5-2C and D).

SUZ12 (suppressor of zeste 12), a Polycomb group (PcG) protein, together with EZH2 (enhancer of zeste homolog 2) and EED (embryonic ectoderm development) form the Polycomb repressive complex, PRC2 [169, 170]. SUZ12 is essential for PRC2 activity to repress transcription through methylation of lysine (K) 27 of histone (H3) (H3K27 methylation). Inactivation of SUZ12 results in early lethality of mouse embryos $[171,172]$. It has been further shown that Suz $12^{-/-}$ESCs failed to undergo proper differentiation upon induction suggesting that SUZ12 is required for the repression of genes essential for ESCs self-renewal, like Nanog and Oct4 [171]. Mcl-1-null ESCs downregulated SUZ12, which is consistent with the differentiation defect phenotype (Figure 5-2C). Future studies need to focus on how MCL-1 and SUZ12 are related to facilitate ESCs differentiation. It is possible that SUZ12 is downstream of MCL-1, thereby regulating the global methylation of H3K27. To test the hypothesis, SUZ12 overexpression experiment could be performed in $\mathrm{Mcl}$-1-deleted or $30 \mathrm{kD}$ mutant ESCs to examine whether restoration of SUZ12 is capable of rescuing the differentiation defect. If SUZ12 is the downstream effector of MCL-1, chromatin immunoprecipitation sequencing (ChIP-Seq) and RNA sequencing (RNA-seq) could be initiated to understand potential genes regulated by epigenetic control via the MCL-1-SUZ12 pathway.

Another pathway identified by GSEA was the upregulated epithelial to mesenchymal transition (EMT) in Mcl-1-deleted ESCs (Figure 5-2D). Consistent with our previous discussion, one study suggested that MCL-1 interacts with STAT3 at the window of implantation to promote the mesenchymal to epithelial transition (MET), essential for successful implantation $[142,143]$. Therefore, it will be also important to test how MCL-1 interacts with STAT3 to facilitate MET for ESCs differentiation and embryo implantation. First, we could evaluate the expression level, subcellular localization and phosphorylation status of STAT3 in the presence or absence of MCL-1. If there is a difference, we could test whether restoration of STAT3 levels rescues the differentiation defect. Furthermore, there are many STAT3 mutants (constitutively active, dominant negative, or catalytic-dead mutant STAT3) that we could use to test how MCL1 and STAT3 interact with each other. 
A

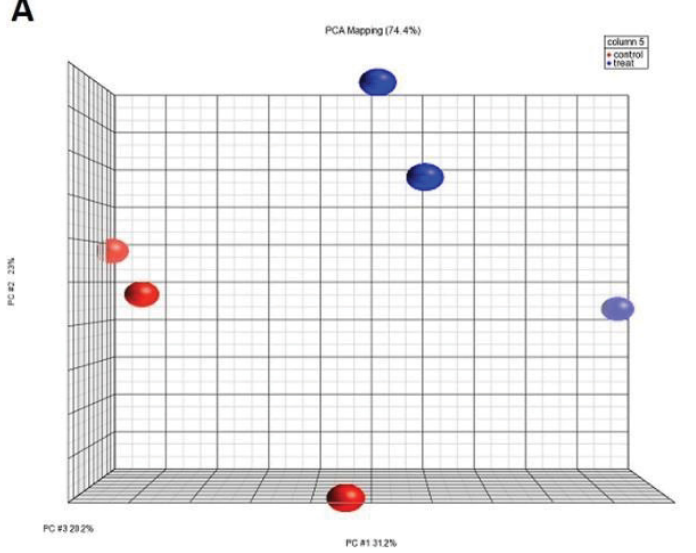

C

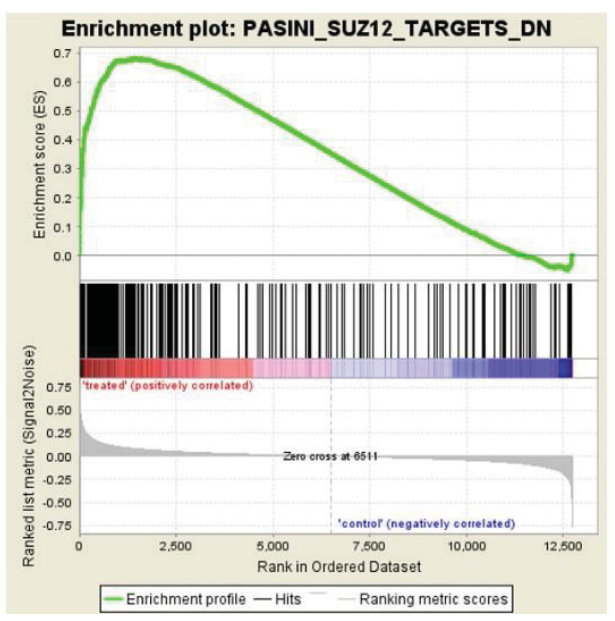

B

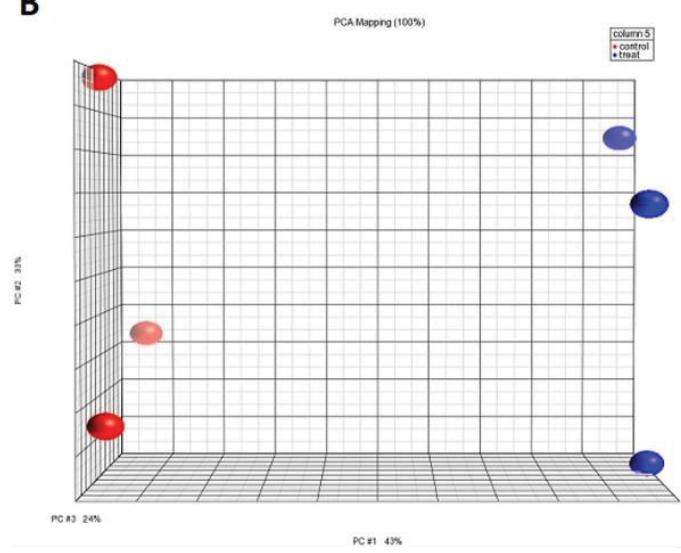

D

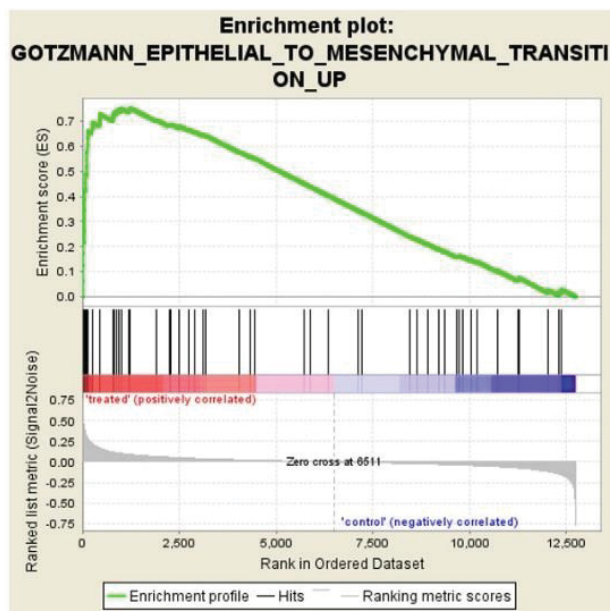

Figure 5-2. Potential changes of signaling pathways are identified in Mcl-1deleted ESCs

(A) Principal components analysis and (B) corrected PCA data comparing Mcl- $1^{\text {flox/flox }}$ $\mathrm{CRE}^{\text {ERT2 }}$ ESCs control and Mcl-1-deleted ESCs. (C) Top $1^{\text {st }}$ and (D) top $4^{\text {th }}$ hits of possible downregulated and upregulated signaling pathways from GSEA by comparing $M c l-1$-deleted ESCs versus $M c l-1^{\text {flox/flox }} \mathrm{CRE}^{\text {ERT2 }}$ ESCs control. $M c l-1^{\text {flox/flox }} \mathrm{CRE}^{\text {ERT2 }}$ ESCs treated with 48 hours tamoxifen were statistically tested by unequal variance $t$ test to the DMSO control (3 replicates). $P$ values for both downregulated SUZ12 and upregulated EMT pathways are 0.000 . 


\section{Determine the Phenotypes of $\mathrm{CS}^{10,11}$ and $30 \mathrm{kD}$ MCL-1 KI Mice}

Our work showed that $\mathrm{CS}^{10,11}$ and $30 \mathrm{kD}$ MCL-1 KI heterozygous were successfully derived. Because of potential off-target effects from CRISPR-Cas9 targeting, our F1 heterozygous mice were backcrossed to WT C57BL/6 mice [173]. After backcrossing, they will be crossbred and the Mendelian ratio will be calculated. Future experiments will set out to try to figure out the phenotypes of $\mathrm{CS}^{10,11}$ and $30 \mathrm{kD} \mathrm{MCL}-1$ KI Mice. To determine whether $\mathrm{CS}^{10,11}$ and $30 \mathrm{kD}$ MCL-1 KI ESCs have defects in differentiation, ESCs from KI homozygous mice will be derived and differentiation assays will be repeated. It will be also interesting to check whether there are any defects in hematopoiesis, cardiac homeostasis, and malignant transformation, where both forms of MCL-1 seem to play important roles. 


\section{LIST OF REFERENCES}

1. Cory, S. and J.M. Adams, The Bcl2 family: regulators of the cellular life-or-death switch. Nat Rev Cancer, 2002. 2(9): p. 647-56.

2. Youle, R.J. and A. Strasser, The BCL-2 protein family: opposing activities that mediate cell death. Nature Reviews Molecular Cell Biology, 2008. 9(1): p. 47-59.

3. Munoz-Pinedo, C., Signaling pathways that regulate life and cell death:

Evolution of Apoptosis in the Context of Self-Defense. Self and Nonself, 2012. 738: p. 124-143.

4. Cheng, E.H.Y.A., et al., BCL-2, BCL-X-L sequester BH3 domain-only molecules preventing $B A X$ - and $B A K$-mediated mitochondrial apoptosis. Molecular Cell, 2001. 8(3): p. 705-711.

5. Petros, A.M., E.T. Olejniczak, and S.W. Fesik, Structural biology of the Bcl-2 family of proteins. Biochimica Et Biophysica Acta-Molecular Cell Research, 2004. 1644(2-3): p. 83-94.

6. Opferman, J.T. and S.J. Korsmeyer, Apoptosis in the development and maintenance of the immune system. Nature Immunology, 2003. 4(5): p. 410-415.

7. Gross, A., J.M. McDonnell, and S.J. Korsmeyer, BCL-2 family members and the mitochondria in apoptosis. Genes \& Development, 1999. 13(15): p. 1899-1911.

8. Kozopas, K.M., et al., Mcl1, a Gene Expressed in Programmed Myeloid CellDifferentiation, Has Sequence Similarity to Bcl2. Proceedings of the National Academy of Sciences of the United States of America, 1993. 90(8): p. 3516-3520.

9. Sattler, M., et al., Structure of Bcl-xL-Bak peptide complex: recognition between regulators of apoptosis. Science, 1997. 275(5302): p. 983-6.

10. Day, C.L., et al., Solution structure of prosurvival Mcl-1 and characterization of its binding by proapoptotic BH3-only ligands. Journal of Biological Chemistry, 2005. 280(6): p. 4738-4744.

11. Veis, D.J., et al., Bcl-2-Deficient Mice Demonstrate Fulminant Lymphoid Apoptosis, Polycystic Kidneys, and Hypopigmented Hair. Cell, 1993. 75(2): p. 229-240.

12. Motoyama, N., et al., Massive Cell-Death of Immature Hematopoietic-Cells and Neurons in Bcl-X-Deficient Mice. Science, 1995. 267(5203): p. 1506-1510. 
13. Print, C.G., et al., Apoptosis regulator Bcl-w is essential for spermatogenesis but appears otherwise redundant. Proceedings of the National Academy of Sciences of the United States of America, 1998. 95(21): p. 12424-12431.

14. Hamasaki, A., et al., Accelerated neutrophil apoptosis in mice lacking A1-a, a subtype of the bcl-2-related A1 gene. Journal of Experimental Medicine, 1998. 188(11): p. 1985-1992.

15. Schenk, R.L., et al., Characterisation of mice lacking all functional isoforms of the pro-survival BCL-2 family member A1 reveals minor defects in the haematopoietic compartment. Cell Death and Differentiation, 2017. 24(3): p. 534545.

16. Rinkenberger, J.L., et al., Mcl-1 deficiency results in peri-implantation embryonic lethality. Genes \& Development, 2000. 14(1): p. 23-27.

17. Opferman, J.T., et al., Development and maintenance of $B$ and T lymphocytes requires antiapoptotic MCL-1. Nature, 2003. 426(6967): p. 671-676.

18. Dzhagalov, I., A. Dunkle, and Y.W. He, The anti-apoptotic Bcl-2 family member Mcl-1 promotes T lymphocyte survival at multiple stages. Journal of Immunology, 2008. 181(1): p. 521-528.

19. Opferman, J.T., et al., Obligate role of anti-apoptotic MCL-1 in the survival of hematopoietic stem cells. Science, 2005. 307(5712): p. 1101-1104.

20. Dzhagalov, I., A. St John, and Y.W. He, The antiapoptotic protein Mcl-1 is essential for the survival of neutrophils but not macrophages. Blood, 2007. 109(4): p. 1620-1626.

21. Steimer, D.A., et al., Selective roles for antiapoptotic MCL-1 during granulocyte development and macrophage effector function. Blood, 2009. 113(12): p. 28052815.

22. Sathe, P., et al., Innate immunodeficiency following genetic ablation of Mcll in natural killer cells. Nature Communications, 2014. 5.

23. Peperzak, V., et al., Mcl-1 is essential for the survival of plasma cells. Nature Immunology, 2013. 14(3): p. 290-297.

24. Motoyama, N., et al., bcl-x prevents apoptotic cell death of both primitive and definitive erythrocytes at the end of maturation. Journal of Experimental Medicine, 1999. 189(11): p. 1691-1698. 
25. Ottina, E., et al., Targeting antiapoptotic A1/Bfl-1 by in vivo RNAi reveals multiple roles in leukocyte development in mice. Blood, 2012. 119(25): p. 60326042.

26. Wang, X., et al., Deletion of MCL-1 causes lethal cardiac failure and mitochondrial dysfunction. Genes \& Development, 2013. 27(12): p. 1351-1364.

27. Thomas, R.L., et al., Loss of MCL-1 leads to impaired autophagy and rapid development of heart failure. Genes \& Development, 2013. 27(12): p. 1365-1377.

28. Arbour, N., et al., Mcl-1 is a key regulator of apoptosis during CNS development and after DNA damage. Journal of Neuroscience, 2008. 28(24): p. 6068-6078.

29. Krajewski, S., et al., Immunohistochemical Analysis of Mcl-1 Protein in Human Tissues - Differential Regulation of Mcl-1 and Bcl-2 Protein-Production Suggests a Unique Role for Mcl-1 in Control of Programmed Cell-Death in-Vivo.

American Journal of Pathology, 1995. 146(6): p. 1309-1319.

30. Sitailo, L.A., A. Jerome-Morais, and M.F. Denning, Mcl-1 Functions as Major Epidermal Survival Protein Required for Proper Keratinocyte Differentiation. Journal of Investigative Dermatology, 2009. 129(6): p. 1351-1360.

31. Beroukhim, R., et al., The landscape of somatic copy-number alteration across human cancers. Nature, 2010. 463(7283): p. 899-905.

32. Holleman, A., et al., Gene-expression patterns in drug-resistant acute lymphoblastic leukemia cells and response to treatment. New England Journal of Medicine, 2004. 351(6): p. 533-542.

33. Wei, G., et al., Gene expression-based chemical genomics identifies rapamycin as a modulator of MCL1 and glucocorticoid resistance. Cancer Cell, 2006. 10(4): p. 331-342.

34. Pepper, C., et al., Mcl-1 expression has in vitro and in vivo significance in chronic lymphocytic leukemia and is associated with other poor prognostic markers. Blood, 2008. 112(9): p. 3807-3817.

35. Kaufmann, S.H., et al., Elevated expression of the apoptotic regulator Mcl-1 at the time of leukemic relapse. Blood, 1998. 91(3): p. 991-1000.

36. Hanahan, D. and R.A. Weinberg, Hallmarks of Cancer: The Next Generation. Cell, 2011. 144(5): p. 646-674.

37. Kotschy, A., et al., The MCL1 inhibitor S63845 is tolerable and effective in diverse cancer models. Nature, 2016. 538(7626): p. 477-+. 
38. Perciavalle, R.M., et al., Anti-apoptotic MCL-1 localizes to the mitochondrial matrix and couples mitochondrial fusion to respiration. Nature Cell Biology, 2012. 14(6): p. 575-+.

39. Huang, C.R. and H.F. Yang-Yen, The fast-mobility isoform of mouse Mcl-1 is a mitochondrial matrix-localized protein with attenuated anti-apoptotic activity. Febs Letters, 2010. 584(15): p. 3323-3330.

40. Perciavalle, R.M. and J.T. Opferman, Delving deeper: MCL-1's contributions to normal and cancer biology. Trends in Cell Biology, 2013. 23(1): p. 22-29.

41. Newmeyer, D.D. and S. Ferguson-Miller, Mitochondria: Releasing power for life and unleashing the machineries of death. Cell, 2003. 112(4): p. 481-490.

42. Wallace, D.C., Mitochondrial diseases in man and mouse. Science, 1999. 283(5407): p. 1482-1488.

43. Wallace, D.C., Mitochondria and cancer. Nature Reviews Cancer, 2012. 12(10): p. 685-698.

44. Detmer, S.A. and D.C. Chan, Functions and dysfunctions of mitochondrial dynamics. Nat Rev Mol Cell Biol, 2007. 8(11): p. 870-9.

45. Detmer, S.A. and D.C. Chan, Complementation between mouse Mfn1 and Mfn2 protects mitochondrial fusion defects caused by CMT2A disease mutations. Journal of Cell Biology, 2007. 176(4): p. 405-414.

46. Meeusen, S., J.M. McCaffery, and J. Nunnari, Mitochondrial fusion intermediates revealed in vitro. Science, 2004. 305(5691): p. 1747-52.

47. Hermann, G.J., et al., Mitochondrial fusion in yeast requires the transmembrane GTPase Fzolp. J Cell Biol, 1998. 143(2): p. 359-73.

48. Koshiba, T., et al., Structural basis of mitochondrial tethering by mitofusin complexes. Science, 2004. 305(5685): p. 858-862.

49. Chen, H.C., A. Chomyn, and D.C. Chan, Disruption of fusion results in mitochondrial heterogeneity and dysfunction. Journal of Biological Chemistry, 2005. 280(28): p. 26185-26192.

50. Meeusen, S., et al., Mitochondrial inner-membrane fusion and crista maintenance requires the dynamin-related GTPase Mgm1. Cell, 2006. 127(2): p. 383-395.

51. Cipolat, S., et al., OPA1 requires mitofusin 1 to promote mitochondrial fusion. Proceedings of the National Academy of Sciences of the United States of America, 2004. 101(45): p. 15927-15932. 
52. Smirnova, E., et al., Dynamin-related protein Drpl is required for mitochondrial division in mammalian cells. Molecular Biology of the Cell, 2001. 12(8): p. 22452256.

53. Nunnari, J., et al., Mitochondrial transmission during mating in Saccharomyces cerevisiae is determined by mitochondrial fusion and fission and the intramitochondrial segregation of mitochondrial DNA. Molecular Biology of the Cell, 1997. 8(7): p. 1233-1242.

54. Chen, H., J.M. McCaffery, and D.C. Chan, Mitochondrial fusion protects against neurodegeneration in the cerebellum. Cell, 2007. 130(3): p. 548-62.

55. Legros, F., et al., Organization and dynamics of human mitochondrial DNA. Journal of Cell Science, 2004. 117(13): p. 2653-2662.

56. Hollenbeck, P.J. and W.M. Saxton, The axonal transport of mitochondria. Journal of Cell Science, 2005. 118(23): p. 5411-5419.

57. Li, Z., et al., The importance of dendritic mitochondria in the morphogenesis and plasticity of spines and synapses. Cell, 2004. 119(6): p. 873-887.

58. Chang, D.T.W., A.S. Honick, and I.J. Reynolds, Mitochondrial trafficking to synapses in cultured primary cortical neurons. Journal of Neuroscience, 2006. 26(26): p. 7035-7045.

59. Chen, H.C., et al., Mitofusins Mfn1 and Mfn2 coordinately regulate mitochondrial fusion and are essential for embryonic development. Journal of Cell Biology, 2003. 160(2): p. 189-200.

60. Alavi, M.V., et al., A splice site mutation in the murine Opal gene features pathology of autosomal dominant optic atrophy. Brain, 2007. 130(Pt 4): p. 102942.

61. Davies, V.J., et al., Opal deficiency in a mouse model of autosomal dominant optic atrophy impairs mitochondrial morphology, optic nerve structure and visual function. Hum Mol Genet, 2007. 16(11): p. 1307-18.

62. Chen, H., et al., Mitofusins Mfn1 and Mfn2 coordinately regulate mitochondrial fusion and are essential for embryonic development. J Cell Biol, 2003. 160(2): p. 189-200.

63. Waterham, H.R., et al., A lethal defect of mitochondrial and peroxisomal fission. N Engl J Med, 2007. 356(17): p. 1736-41.

64. Wilding, M., et al., Mitochondria and human preimplantation embryo development. Reproduction, 2009. 137(4): p. 619-24. 
65. McBride, H.M., M. Neuspiel, and S. Wasiak, Mitochondria: More than just a powerhouse. Current Biology, 2006. 16(14): p. R551-R560.

66. Tuppen, H.A.L., et al., Mitochondrial DNA mutations and human disease. Biochimica Et Biophysica Acta-Bioenergetics, 2010. 1797(2): p. 113-128.

67. Novotny, E.J., Jr., et al., Leber's disease and dystonia: a mitochondrial disease. Neurology, 1986. 36(8): p. 1053-60.

68. Wallace, D.C., et al., Mitochondrial DNA mutation associated with Leber's hereditary optic neuropathy. Science, 1988. 242(4884): p. 1427-30.

69. Holt, I.J., A.E. Harding, and J.A. Morganhughes, Deletions of Muscle Mitochondrial-DNA in Patients with Mitochondrial Myopathies. Nature, 1988. 331(6158): p. 717-719.

70. Ballinger, S.W., et al., Mitochondrial Diabetes Revisited. Nature Genetics, 1994. 7(4): p. 458-459.

71. Heddi, A., et al., Steady-State Levels of Mitochondrial and Nuclear OxidativePhosphorylation Transcripts in Kearns-Sayre Syndrome. Biochimica Et Biophysica Acta-Molecular Basis of Disease, 1994. 1226(2): p. 206-212.

72. Heddi, A., et al., Mitochondrial-DNA Expression in Mitochondrial Myopathies and Coordinated Expression of Nuclear Genes Involved in Atp Production. Journal of Biological Chemistry, 1993. 268(16): p. 12156-12163.

73. Smeitink, J. and L. van den Heuvel, Human mitochondrial complex I in health and disease. American Journal of Human Genetics, 1999. 64(6): p. 1505-1510.

74. Rotig, A., et al., Aconitase and mitochondrial iron-sulphur protein deficiency in Friedreich ataxia. Nature Genetics, 1997. 17(2): p. 215-217.

75. Fulda, S., L. Galluzzi, and G. Kroemer, Targeting mitochondria for cancer therapy. Nature Reviews Drug Discovery, 2010. 9(6): p. 447-464.

76. Canter, J.A., et al., Mitochondrial DNA G10398A polymorphism and invasive breast cancer in African-American women. Cancer Research, 2005. 65(17): p. 8028-8033.

77. Permuth-Wey, J., et al., Inherited Variants in Mitochondrial Biogenesis Genes May Influence Epithelial Ovarian Cancer Risk. Cancer Epidemiology Biomarkers \& Prevention, 2011. 20(6): p. 1131-1145.

78. Vyas, S., E. Zaganjor, and M.C. Haigis, Mitochondria and Cancer. Cell, 2016. 166(3): p. 555-566. 
79. Hay, N. and N. Sonenberg, Upstream and downstream of mTOR. Genes \& Development, 2004. 18(16): p. 1926-1945.

80. Schieber, M. and N.S. Chandel, ROS Function in Redox Signaling and Oxidative Stress. Current Biology, 2014. 24(10): p. R453-R462.

81. Budanov, A.V., et al., Regeneration of peroxiredoxins by p53-regulated sestrins, homologs of bacterial AhpD. Science, 2004. 304(5670): p. 596-600.

82. Sablina, A.A., et al., The antioxidant function of the p53 tumor suppressor. Nature Medicine, 2005. 11(12): p. 1306-1313.

83. Li, T.Y., et al., Tumor Suppression in the Absence of p53-Mediated Cell-Cycle Arrest, Apoptosis, and Senescence. Cell, 2012. 149(6): p. 1269-1283.

84. Sullivan, L.B. and N.S. Chandel, Mitochondrial reactive oxygen species and cancer. Cancer Metab, 2014. 2: p. 17.

85. Porporato, P.E., et al., A mitochondrial switch promotes tumor metastasis. Cell Rep, 2014. 8(3): p. 754-66.

86. Zamora, M., et al., Adenine nucleotide translocase 3 (ANT3) overexpression induces apoptosis in cultured cells. Febs Letters, 2004. 563(1-3): p. 155-160.

87. Bauer, M.K.A., et al., Adenine nucleotide translocase-1, a component of the permeability transition pore, can dominantly induce apoptosis. Journal of Cell Biology, 1999. 147(7): p. 1493-1501.

88. Le Bras, M., et al., Chemosensitization by knockdown of adenine nucleotide translocase-2. Cancer Research, 2006. 66(18): p. 9143-9152.

89. Marzo, I., et al., Bax and adenine nucleotide translocator cooperate in the mitochondrial control of apoptosis. Science, 1998. 281(5385): p. 2027-2031.

90. Don, A.S., et al., A peptide trivalent arsenical inhibits tumor angiogenesis by perturbing mitochondrial function in angiogenic endothelial cells. Cancer Cell, 2003. 3(5): p. 497-509.

91. Belzacq, A.S., et al., Adenine nucleotide translocator mediates the mitochondrial membrane permeabilization induced by lonidamine, arsenite and CD437. Oncogene, 2001. 20(52): p. 7579-7587.

92. Magda, D. and R.A. Miller, Motexafin gadolinium: A novel redox active drug for cancer therapy. Seminars in Cancer Biology, 2006. 16(6): p. 466-476. 
93. Bradley, K.A., et al., Motexafin gadolinium and involved field radiation therapy for intrinsic pontine glioma of childhood: A Children's Oncology Group phase I study. Neuro-Oncology, 2008. 10(5): p. 752-758.

94. Oltersdorf, T., et al., An inhibitor of Bcl-2 family proteins induces regression of solid tumours. Nature, 2005. 435(7042): p. 677-681.

95. Konopleva, M., et al., Mechanisms of apoptosis sensitivity and resistance to the BH3 mimetic ABT-737 in acute myeloid leukemia. Annals of Oncology, 2006. 17.

96. Mason, K.D., et al., In vivo efficacy of the Bcl-2 antagonist ABT-737 against aggressive Myc-driven lymphomas. Proceedings of the National Academy of Sciences of the United States of America, 2008. 105(46): p. 17961-17966.

97. Simons, A.L., et al., 2-Deoxy-D-glucose combined with cisplatin, enhances cytotoxicity via metabolic oxidative stress in human head and neck cancer cells. Cancer Research, 2007. 67(7): p. 3364-3370.

98. Hsu, P.D., E.S. Lander, and F. Zhang, Development and applications of CRISPRCas 9 for genome engineering. Cell, 2014. 157(6): p. 1262-78.

99. Yin, H., K.J. Kauffman, and D.G. Anderson, Delivery technologies for genome editing. Nat Rev Drug Discov, 2017. 16(6): p. 387-399.

100. Doudna, J.A. and E. Charpentier, Genome editing. The new frontier of genome engineering with CRISPR-Cas9. Science, 2014. 346(6213): p. 1258096.

101. Stoddard, B.L., Homing Endonucleases: From Microbial Genetic Invaders to Reagents for Targeted DNA Modification. Structure, 2011. 19(1): p. 7-15.

102. Urnov, F.D., et al., Genome editing with engineered zinc finger nucleases. Nature Reviews Genetics, 2010. 11(9): p. 636-646.

103. Joung, J.K. and J.D. Sander, INNOVATION TALENs: a widely applicable technology for targeted genome editing. Nature Reviews Molecular Cell Biology, 2013. 14(1): p. 49-55.

104. Ran, F.A., et al., Genome engineering using the CRISPR-Cas9 system. Nature Protocols, 2013. 8(11): p. 2281-2308.

105. Wu, Y.X., et al., Correction of a Genetic Disease in Mouse via Use of CRISPRCas9. Cell Stem Cell, 2013. 13(6): p. 659-662.

106. Schwank, G., et al., Functional Repair of CFTR by CRISPR/Cas9 in Intestinal Stem Cell Organoids of Cystic Fibrosis Patients. Cell Stem Cell, 2013. 13(6): p. 653-658. 
107. Wang, T., et al., Genetic Screens in Human Cells Using the CRISPR-Cas9 System. Science, 2014. 343(6166): p. 80-84.

108. Shalem, O., et al., Genome-Scale CRISPR-Cas9 Knockout Screening in Human Cells. Science, 2014. 343(6166): p. 84-87.

109. Tebas, P., et al., Gene Editing of CCR5 in Autologous CD4 T Cells of Persons Infected with HIV. New England Journal of Medicine, 2014. 370(10): p. 901-910.

110. Koss, B., et al., Requirement for antiapoptotic $M C L-1$ in the survival of $B C R-A B L$ B-lineage acute lymphoblastic leukemia. Blood, 2013. 122(9): p. 1587-98.

111. Richardson, C.D., et al., Enhancing homology-directed genome editing by catalytically active and inactive CRISPR-Cas9 using asymmetric donor DNA. Nature Biotechnology, 2016. 34(3): p. 339-+.

112. Vanblerkom, J., P.W. Davis, and J. Lee, Atp Content of Human Oocytes and Developmental Potential and Outcome after in-Vitro Fertilization and EmbryoTransfer. Human Reproduction, 1995. 10(2): p. 415-424.

113. Motta, P.M., et al., Mitochondrial morphology in human fetal and adult female germ cells. Hum Reprod, 2000. 15 Suppl 2: p. 129-47.

114. Dumollard, R., M. Duchen, and J. Carroll, The role of mitochondrial function in the oocyte and embryo. Curr Top Dev Biol, 2007. 77: p. 21-49.

115. Larsson, N.G., et al., Mitochondrial transcription factor A is necessary for mtDNA maintenance and embryogenesis in mice. Nature Genetics, 1998. 18(3): p. 231-236.

116. Van Blerkom, J., Morphodynamics of nuclear and cytoplasmic reorganization during the resumption of arrested meiosis in the mouse oocyte. Prog Clin Biol Res, 1989. 294: p. 33-51.

117. Vanblerkom, J., Development of Human Embryos to the Hatched Blastocyst Stage in the Presence or Absence of a Monolayer of Vero Cells. Human Reproduction, 1993. 8(9): p. 1525-1539.

118. Sathananthan, A.H. and A.O. Trounson, Mitochondrial morphology during preimplantational human embryogenesis. Hum Reprod, 2000. 15 Suppl 2: p. 14859.

119. Johnson, M.T., S. Mahmood, and M.S. Patel, Intermediary metabolism and energetics during murine early embryogenesis. Journal of Biological Chemistry, 2003. 278(34): p. 31457-31460. 
120. Van Blerkom, J., Mitochondria in early mammalian development. Seminars in Cell \& Developmental Biology, 2009. 20(3): p. 354-364.

121. Piccoli, C., et al., Characterization of mitochondrial and extra-mitochondrial oxygen consuming reactions in human hematopoietic stem cells. Novel evidence of the occurrence of $N A D(P) H$ oxidase activity. J Biol Chem, 2005. 280(28): p. 26467-76.

122. Lonergan, T., B. Bavister, and C. Brenner, Mitochondria in stem cells. Mitochondrion, 2007. 7(5): p. 289-96.

123. Chen, C.T., et al., Coordinated changes of mitochondrial biogenesis and antioxidant enzymes during osteogenic differentiation of human mesenchymal stem cells. Stem Cells, 2008. 26(4): p. 960-8.

124. Vander Heiden, M.G., L.C. Cantley, and C.B. Thompson, Understanding the Warburg effect: the metabolic requirements of cell proliferation. Science, 2009. 324(5930): p. 1029-33.

125. Zhang, J., et al., Metabolic regulation in pluripotent stem cells during reprogramming and self-renewal. Cell Stem Cell, 2012. 11(5): p. 589-95.

126. Mohyeldin, A., T. Garzon-Muvdi, and A. Quinones-Hinojosa, Oxygen in stem cell biology: a critical component of the stem cell niche. Cell Stem Cell, 2010. 7(2): p. 150-61.

127. Varum, S., et al., Enhancement of human embryonic stem cell pluripotency through inhibition of the mitochondrial respiratory chain. Stem Cell Research, 2009. 3(2-3): p. 142-156.

128. Kondoh, H., et al., A high glycolytic flux supports the proliferative potential of murine embryonic stem cells. Antioxid Redox Signal, 2007. 9(3): p. 293-9.

129. Folmes, C.D., et al., Somatic oxidative bioenergetics transitions into pluripotency-dependent glycolysis to facilitate nuclear reprogramming. Cell Metab, 2011. 14(2): p. 264-71.

130. Chung, S., et al., Mitochondrial oxidative metabolism is required for the cardiac differentiation of stem cells. Nat Clin Pract Cardiovasc Med, 2007. 4 Suppl 1: p. S60-7.

131. Lonergan, T., C. Brenner, and B. Bavister, Differentiation-related changes in mitochondrial properties as indicators of stem cell competence. J Cell Physiol, 2006. 208(1): p. 149-53. 
132. Tormos, K.V., et al., Mitochondrial Complex III ROS Regulate Adipocyte Differentiation. Cell Metabolism, 2011. 14(4): p. 537-544.

133. Hom, J.R., et al., The Permeability Transition Pore Controls Cardiac Mitochondrial Maturation and Myocyte Differentiation. Developmental Cell, 2011. 21(3): p. 469-478.

134. Owusu-Ansah, E. and U. Banerjee, Reactive oxygen species prime Drosophila haematopoietic progenitors for differentiation. Nature, 2009. 461(7263): p. $537-$ 41.

135. Zhang, J., et al., UCP2 regulates energy metabolism and differentiation potential of human pluripotent stem cells. EMBO J, 2011. 30(24): p. 4860-73.

136. Niwa, H., et al., Self-renewal of pluripotent embryonic stem cells is mediated via activation of STAT3. Genes \& Development, 1998. 12(13): p. 2048-2060.

137. Ying, Q.L., et al., BMP induction of Id proteins suppresses differentiation and sustains embryonic stem cell self-renewal in collaboration with STAT3. Cell, 2003. 115(3): p. 281-292.

138. Chen, C., et al., TSC-mTOR maintains quiescence and function of hematopoietic stem cells by repressing mitochondrial biogenesis and reactive oxygen species. Journal of Experimental Medicine, 2008. 205(10): p. 2397-2408.

139. Kentsis, A. and A.T. Look, Distinct and Dynamic Requirements for mTOR Signaling in Hematopoiesis and Leukemogenesis. Cell Stem Cell, 2012. 11(3): p. 281-282.

140. Valcourt, J.R., et al., Staying alive Metabolic adaptations to quiescence. Cell Cycle, 2012. 11(9): p. 1680-1696.

141. Moussaieff, A., et al., Glycolysis-Mediated Changes in Acetyl-CoA and Histone Acetylation Control the Early Differentiation of Embryonic Stem Cells. Cell Metabolism, 2015. 21(3): p. 392-402.

142. Renjini, A.P., et al., STAT3 and MCL-1 associate to cause a mesenchymal epithelial transition. Journal of Cell Science, 2014. 127(8): p. 1738-1750.

143. Zhang, X.H., et al., The Mesenchymal-Epithelial Transition During In Vitro Decidualization. Reproductive Sciences, 2013. 20(4): p. 354-360.

144. Xin, M., E.N. Olson, and R. Bassel-Duby, Mending broken hearts: cardiac development as a basis for adult heart regeneration and repair. Nature Reviews Molecular Cell Biology, 2013. 14(8): p. 529-541. 
145. Folmes, C.D.L., et al., Mitochondria in Control of Cell Fate. Circulation Research, 2012. 110(4): p. 526-529.

146. Porrello, E.R., et al., Transient Regenerative Potential of the Neonatal Mouse Heart. Science, 2011. 331(6020): p. 1078-1080.

147. Wencker, D., et al., A mechanistic role for cardiac myocyte apoptosis in heart failure. Journal of Clinical Investigation, 2003. 111(10): p. 1497-1504.

148. Opferman, J.T., Life and death during hematopoietic differentiation. Current Opinion in Immunology, 2007. 19(5): p. 497-502.

149. Certo, M., et al., Mitochondria primed by death signals determine cellular addiction to antiapoptotic BCL-2 family members. Cancer Cell, 2006. 9(5): p. 351-365.

150. van Delft, M.F., et al., The BH3 mimetic ABT-737 targets selective Bcl-2 proteins and efficiently induces apoptosis via Bak/Bax if Mcl-1 is neutralized. Cancer Cell, 2006. 10(5): p. 389-399.

151. Rudin, C.M., et al., Phase II Study of Single-Agent Navitoclax (ABT-263) and Biomarker Correlates in Patients with Relapsed Small Cell Lung Cancer. Clinical Cancer Research, 2012. 18(11): p. 3163-3169.

152. Souers, A.J., et al., ABT-199, a potent and selective BCL-2 inhibitor, achieves antitumor activity while sparing platelets. Nature Medicine, 2013. 19(2): p. 202208.

153. Pan, R., et al., Selective BCL-2 inhibition by ABT-199 causes on-target cell death in acute myeloid leukemia. Cancer Discov, 2014. 4(3): p. 362-75.

154. Vandenberg, C.J. and S. Cory, ABT-199, a new Bcl-2-specific BH3 mimetic, has in vivo efficacy against aggressive Myc-driven mouse lymphomas without provoking thrombocytopenia. Blood, 2013. 121(12): p. 2285-8.

155. Peirs, S., et al., ABT-199 mediated inhibition of BCL-2 as a novel therapeutic strategy in T-cell acute lymphoblastic leukemia. Blood, 2014. 124(25): p. 373847.

156. Roberts, A.W., et al., Targeting BCL2 with Venetoclax in Relapsed Chronic Lymphocytic Leukemia. N Engl J Med, 2016. 374(4): p. 311-22.

157. Seymour, J.F., et al., Venetoclax plus rituximab in relapsed or refractory chronic lymphocytic leukaemia: a phase 1b study. Lancet Oncol, 2017. 18(2): p. 230-240. 
158. Davids, M.S., et al., Phase I First-in-Human Study of Venetoclax in Patients With Relapsed or Refractory Non-Hodgkin Lymphoma. J Clin Oncol, 2017. 35(8): p. 826-833.

159. Visvader, J.E. and G.J. Lindeman, Cancer stem cells: current status and evolving complexities. Cell Stem Cell, 2012. 10(6): p. 717-28.

160. Hermann, P.C., et al., Distinct populations of cancer stem cells determine tumor growth and metastatic activity in human pancreatic cancer. Cell Stem Cell, 2007. 1(3): p. 313-23.

161. Miranda-Lorenzo, I., et al., Intracellular autofluorescence: a biomarker for epithelial cancer stem cells. Nat Methods, 2014. 11(11): p. 1161-9.

162. Dong, C., et al., Loss of FBP1 by Snail-mediated repression provides metabolic advantages in basal-like breast cancer. Cancer Cell, 2013. 23(3): p. 316-31.

163. Shen, Y.A., et al., Metabolic reprogramming orchestrates cancer stem cell properties in nasopharyngeal carcinoma. Cell Cycle, 2015. 14(1): p. 86-98.

164. Chen, C.L., et al., NANOG Metabolically Reprograms Tumor-Initiating Stem-like Cells through Tumorigenic Changes in Oxidative Phosphorylation and Fatty Acid Metabolism. Cell Metab, 2016. 23(1): p. 206-19.

165. Debrincat, M.A., et al., Mcl-1 and Bcl-x(L) coordinately regulate megakaryocyte survival. Blood, 2012. 119(24): p. 5850-8.

166. Josefsson, E.C., et al., Megakaryocytes possess a functional intrinsic apoptosis pathway that must be restrained to survive and produce platelets. J Exp Med, 2011. 208(10): p. 2017-31.

167. Kim, J.H., et al., High cleavage efficiency of a $2 \mathrm{~A}$ peptide derived from porcine teschovirus-1 in human cell lines, zebrafish and mice. PLoS One, 2011. 6(4): p. e18556.

168. Raychaudhuri, S., J.M. Stuart, and R.B. Altman, Principal components analysis to summarize microarray experiments: application to sporulation time series. Pac Symp Biocomput, 2000: p. 455-66.

169. Cao, R. and Y. Zhang, The functions of E(Z)/EZH2-mediated methylation of lysine 27 in histone H3. Curr Opin Genet Dev, 2004. 14(2): p. 155-64.

170. Kuzmichev, A., et al., Different EZH2-containing complexes target methylation of histone H1 or nucleosomal histone H3. Mol Cell, 2004. 14(2): p. 183-93. 
171. Pasini, D., et al., The polycomb group protein Suz12 is required for embryonic stem cell differentiation. Mol Cell Biol, 2007. 27(10): p. 3769-79.

172. Pasini, D., et al., Suz12 is essential for mouse development and for EZH2 histone methyltransferase activity. EMBO J, 2004. 23(20): p. 4061-71.

173. Cho, S.W., et al., Analysis of off-target effects of CRISPR/Cas-derived RNAguided endonucleases and nickases. Genome Res, 2014. 24(1): p. 132-41. 


\section{VITA}

Xue Yang was born in Wuhan, Hubei, China in 1984. In July of 2009, she completed a unified program for Bachelor Degree and Master Degree of Medicine in Tongji Medical College at Huazhong University of Science and Technology. She enrolled in the Ph.D program at the University of Tennessee Health Science Center in 2011 and joined Dr. Joseph Opferman's laboratory at St. Jude Children's Research Hospital in 2012. On November 18, 2013 she married Zailiang Qin. She is expected to graduate in 2017.

\section{Publications}

JG van Oosterwijk, CL Li, X Yang, JT Opferman, CJ Sherr. 2017. Small mitochondrial Arf (smArf) protein corrects p53-independent developmental defects of Arf tumor suppressor-deficient mice. Proceedings of the National Academy of Sciences 114: 74207425.

D Yin1, XY Xia, JL Zhang, SY Zhang1, F Liao, G Zhang, Y Zhang, QQ Hou, X Yang, H Wang, ZG Ma, HY Wang, YP Zhu, W Zhang, YL Wang, B Liu, LL Wang, H Xu. 2017. Impact of NUDT15 polymorphisms on thiopurines-induced myelotoxicity and thiopurines tolerance dose. Oncotarget. 8: 13575-13585.

B Koss, J Ryan, A Budhraja, K Szarama, X Yang, M Bathina, MH Cardone, Z Nikolovska-Coleska, A Letai, JT Opferman. 2016. Defining specificity and on-target activity of BH3-mimetics using engineered B-ALL cell lines. Oncotarget 7: 1150011511.

YN Zhou, RL Dai, L Mao, YP Xia, YF Yao, X Yang, B Hu. 2010. Activation of sonic hedgehog signaling pathway in S-type neuroblastoma cell lines. Journal of Huazhong University of Science and Technology [Medical Sciences] 30: 271-277.

L Mao, YP Xia, YN Zhou, RL Dai, X Yang, YJ Wang, SJ Duan, X Qiao, YW Mei, B $\mathrm{Hu}$. 2009. Activation of sonic hedgehog signaling pathway in olfactory neuroblastoma. Oncology 77: 231-243.

L Mao, YP Xia, YN Zhou, RL Dai, X Yang, SJ Duan, X Qiao, YW Mei, B Hu, HJ Cui. 2009. A critical role of Sonic Hedgehog signaling in maintaining the tumorigenicity of neuroblastoma cells. Cancer science 100: 1848-1855. 